PALEO

Revue d'archéologie préhistorique

$21 \mid 2009-2010$

Varia

\title{
La fonction des pièces de la Bertonne : un problème en partie résolu
}

Lucie Chehmana, Nicolas Holzem, Jacques Pelegrin et Pierre Bazin

\section{(2) OpenEdition}

Journals

Édition électronique

URL : http://journals.openedition.org/paleo/1750

DOI : $10.4000 /$ paleo. 1750

ISSN : 2101-0420

Éditeur

SAMRA

Édition imprimée

Date de publication : 1 mars 2011

Pagination : 65-102

ISSN : 1145-3370

\section{Référence électronique}

Lucie Chehmana, Nicolas Holzem, Jacques Pelegrin et Pierre Bazin, «La fonction des pièces de la Bertonne : un problème en partie résolu », PALEO [En ligne], 21 | 2009-2010, mis en ligne le 21 octobre 2011, consulté le 07 juillet 2020. URL : http://journals.openedition.org/paleo/1750 ; DOI : https:// doi.org/10.4000/paleo.1750

\section{(c) (i) (9)}

PALEO est mis à disposition selon les termes de la licence Creative Commons Attribution - Pas d'Utilisation Commerciale - Pas de Modification 4.0 International. 


\title{
LA FONCTION DES PIÈCES DE LA BERTONNE : un problème en partie résolu
}

\author{
Lucie CHEHMANA ${ }^{(1)}$, Nicolas HOLZEM(2), \\ Jacques PELEGRIN( ${ }^{(3)}$ et Pierre BAZIN(4)
}

\begin{abstract}
Résumé : Les pièces de la Bertonne sont si particulières qu'il nous a semblé utile d'exposer ici l'ensemble de la démarche qui a permis de déterminer leur véritable fonction. L'argumentation a été essentiellement construite sur la base d'un raisonnement déductif. Les trois hypothèses fonctionnelles (pièce amincie en vue d'un emmanchement, outil et nucléus à lamelles) évoquées depuis leur découverte ont été testées au fur et à mesure dans le but d'éliminer celles qui étaient improbables. La fonction de nucléus à lamelles s'est alors révélée la plus vraisemblable. Ce résultat fut ensuite confirmé par la découverte récente de quelques lamelles retouchées indubitablement obtenues à partir de ces pièces de la Bertonne. Cet article est enfin consacré à la présentation de la méthode originale de débitage de lamelles de type «Bertonne » qui présente de nombreuses analogies avec celle connue sous le nom d’Orville.
\end{abstract}

Mots-clés : Pièces de la Bertonne, pièces d'Orville, Badegoulien, caractérisation fonctionnelle, nucléus à lamelles, méthode de débitage.

Key-words: La Bertonne artifacts, Orville artifacts, Badegoulian, functional description, bladelets cores, reduction strategy.

\section{Abridged english version}

The function of La Bertonne artifacts: a problem partially solved.

La Bertonne artifacts are so specific that it appears necessary to present the methodology that has made it possible to determine their true function. The argument is essentially based on deductive reasoning. The three functional hypotheses proposed since their discovery have been tested in order to eliminate those that are improbable. Using drawing models in addition to use-wear and technological analyses, it was possible to determine whether La Bertonne artifacts were retouched:

- to be hafted,

- to create an active tool part,

- or to produce bladelets.

The function as bladelet cores has been shown to be the most likely. This result was subsequently confirmed by the discovery of retouched bladelets unquestionably obtained from these La Bertonne artifacts. Finally, this article focuses on presenting the original method of La Bertonne bladelet technology, which has several characteristics in common with the Orville method.

Description of La Bertonne artifacts (fig. 1 and 2)

(1) Université de Paris I - UMR 7041 ArScAn, équipe d'ethnologie préhistorique, 21 allée de l'université, F-92023 Nanterre cedex lucie.chehmana@mae.u-paris10.fr

(2) INRAP, centre archéologique de Tours, 148 avenue Maginot, 37100 Tours - nicolas.holzem@inrap.fr

(3) UMR 7055 « Préhistoire et Technologie », MAE Nanterre, 21 allée de l'Université, F-92023 Nanterre cedex -

jacques.pelegrin@mae.u-paris10.fr

(4) L'espérance, 45500 Poilly-les-Gien 
Briefly, four criteria (fig. 2) should be met to identify an artifact as La Bertonne:

- First, the blank should generally be a blade, to which we add a criterion of thinness,

- Second, these artifacts have an inverse truncation,

- Thirdly, this inverse truncation is overlapped by one or more bladelet removal scars, which become increasingly planar,

- Fourthly, the bladelet scars begin quasi-systematically from the edge to the right of the inverse truncation when this is oriented toward the top (that is, the left edge of the support if the truncation is distal, the right edge if the truncation is proximal).

Geographic location of assemblages with La Bertonne artifacts (fig. 3)

During his research, M. Lenoir made an inventory of all of the assemblages with La Bertonne artifacts (Lenoir 1987). Until the beginning of the 1970s, these were mainly found in Southwest France, in the departments of the Gironde and the Dordogne (fig. 3). During the 1970s and 1980s, other sites were identified in neighboring departments, such as Les Landes, the Corrèze and Charente-Maritime, but also somewhat to the north, in the department of La Creuse. Several assemblages were recently located in an even more northern region (Valentin 1995 p. 65), along the Loire Valley. These result from surface collections carried out by one of the authors (P.B.) east of the town of Gien (Loiret). Finally, based on a note by F. Daleau (1910 p. 276), similar artifacts were also found in Haute-Normandie. We attempted to recover this collection from the place-name La Caboche at Saint-Pierre-du-Vauvray (Eure), but unfortunately were informed that this collection had burned during World War II. Regardless, since the first discovery of La Bertonne artifacts, it appears that the area of distribution for these assemblages is much broader than initially believed. We now have evidence that this cultural phenomenon was not limited to a single region, but was widely spread from the Adour Basin to the Loire Valley.

Appearance of La Bertonne artifacts (fig. 4-8; table 1)

The chronological position of La Bertonne artifacts is relatively unclear. Mainly discovered at open-air sites, the assemblages, although numerous, cannot be dated. Connections to other types of industry which are better dated were, however, able to be made. First associated with the Aurignacian (Leysalles \& Noone 1949 ; D. Peyrony 1949 p. 30), they were subsequently attributed to the Badegoulian (Lenoir 2000), formerly termed the Early Magdalenian (Lenoir 1987) or Magdalenian 0 and I (Bordes 1958). A few La Bertonne artifacts were found in stratigraphic context at Laugerie-Haute, in layers I' and I? of the Peyrony excavations (Leysalles \& Noone 1949 p. 250-251) (fig. 4), and also in layers 4 and 10 of the Bordes excavations (Hemingway 1980 p. 324, 335; Demars \& Laurent 1989 p. 88-89 ; Lenoir 1987) (fig. 5). It is at present impossible to date their appearance more precisely or to evaluate the duration of time during which the Badegoulians made La Bertonne artifacts.

Representativeness of the inventoried archaeological sample (fig. 9; table 2)

Of the five assemblages still available, each containing a large number of La Bertonne artifacts, we decided to exhaustively analyze the Trocadero assemblage, which is still unpublished. It contains more than 60 La Bertonne artifacts. Our reasoning is primarily based on this analysis, sometimes complemented, when necessary, with the analysis of artifacts from the eponymous site of La Bertonne. We have also taken into account the collections of la Malignière and Bellevue. We discovered retouched bladelets for which the blanks were clearly obtained from La Bertonne artifacts. The assemblages from Signal and Seyresse, by contrast, could not be examined, but it was not necessary to carry out an exhaustive study of all of these assemblages to resolve the questions proposed.

We also take this opportunity to report the recent discovery of an isolated La Bertonne artifacts (fig. 9), a surface find in front of the site of La Rochette (Saint Léon-sur-Vézère, Dordogne), where no Badegoulian occupation had previously been identified (Movius in Bricker 1995 p. 292).

- First hypothesis: were modifications of « La Bertonne » type made in preparation for hafting? (fig. 11-14; table 3)

It is principally the presence of an active extremity (fig. 11), generally a burin or endscraper, on the part opposite to that with La Bertonne modifications, which has led to research on their function. Was the objective of the Badegoulians to thin these extremities in order to insert them into a haft (fig. 11a)?

In this view, it appeared curious that the knappers had planned asymmetric shaping with respect to the longitudinal axis of the artifact (fig. 11b), more exactly with respect to the axis determined by the positioning of the assumed active part. This initial observation alone did not allow rejection of this hypothesis, but led us to look for other possible evidence incompatible with this function.

We had doubted the validity of this hypothesis from the start, given that in the Trocadero collection these active extremities are present only on $20 \%$ of artifacts that have La Bertonne shaping (table 2). Their presence is thus not systematic. Moreover, there are archaeological examples in which the part opposite to this active extremity was used to extract bladelets. The most well-known examples are the «La Marche » cores (Alix et al. 1995; Chehmana in press). Laminar blanks are thus sometimes reused after having been used as tools. 
Second, we were also able to rapidly exclude this hypothesis because the assemblage contains many double La Bertonne artifacts ( $n=14$; fig. 12a). This means that the same type of shaping was made on both ends of the blades. We considered whether the Badegoulians could have possibly planned tools hafted on two sides (fig. 12b). In this case, the sharp edges would be the only active parts remaining visible. Our attempt at modeling through drawing (fig. 12b), however, demonstrates that such double hafting is not at all ergonomic. In addition, if the sharp edges of the blades had been viewed as the active parts, why would the Badegoulians have used such short edges when they very often produced long blades? At the site of Trocadero, for example, located near several flint outcrops, blades were produced measuring $10-15 \mathrm{~cm}$ long. The double La Bertonne artifacts only leave at maximum $4.5 \mathrm{~cm}$ of exposed edge.

Third, some La Bertonne artifacts still have a wide and protuberant shouldered platform on one of the ends. When we observe the modeling (fig. 13), it is difficult to imagine that the opposite part had been shaped in preparation for hafting! In the opposite situation, the active parts could once again only be the sharp edges of the blades. This hypothesis is seen as entirely unsuitable when we note that some of these preparations were made on flakes which, if hafted (fig. 14), would have been nearly entirely covered by the haft. Moreover, if this thinning had been truly done in preparation for hafting, why do so in many cases (45\% at the Trocadero site) on the distal part of the blank when during the Upper Paleolithic, this part was often used as the active end?

So, based on these different arguments, it is, in our view, improbable that these blade ends were retouched to be inserted into a haft. There remain two other hypotheses to test: use as tool or as bladelet core.

- Second hypothesis: did La Bertonne retouch form an active part? (fig. 15-18)

Use-wear analysis was carried out by one of the authors (N.H.) on a dozen artifacts selected from the Trocadero collection. Our original objective was to evaluate the state of preservation of use-wear polishes and to identify the possible active parts. Although the artifacts are covered with an opaque patina, traces resulting from use were preserved on some of them. The results obtained are thus encouraging. Briefly, no use-wear polish was detected on the ventral faces of La Bertonne artifacts, more specifically where the bladelet scars are found (fig. 15b). By contrast, their presence is observed on the dorsal face on two artifacts. This was seen on a highly localized surface: along the line delimited by the start of the bladelet scars (fig. 15a). The relatively good state of preservation of certain polishes has even permitted determination on one of these pieces of traces linked to contact with hard animal material.

Based on these initial results, we could consider the possible positioning of the tool on the surface to be worked, the action, gesture of use and finally the most probable type of tool. After locating use-wear traces, if these are really tools, the artifacts would rather be position with the dorsal face on the surface to be worked (fig. 16). The action would be scraping. The work would have been done transversal to the edge - forming the line between the dorsal and ventral faces of the tool, at the spot where the bladelet scars began - and probably by negative cutting (fig. 16), meaning towards the user. The presence of splintering only on the ventral face (fig. 17) would not be incompatible with this type of use. The hypothesis of «inverse endscraper » proposed by M. Lenoir cannot be rejected.

We have, however, observed on several examples (fig. 18-21) that no splintering exists on the departure line of the last bladelet scar. This indicates that these pieces were not used after the last modification and suggests the hypothesis that at least this final action was done in the aim of producing a bladelet.

- Third hypothesis: did the modifications result from bladelet production? (fig. 19-23)

To test this hypothesis, we needed to find what could correspond to the possible objective of this production, that is, retouched bladelets that would have been unquestionably detached from La Bertonne artifacts. It has, however, often been observed that none of the collection with La Bertonne artifacts have retouched bladelets. None except one, that of La Malignière! Given that this collection is composed of several archaeological assemblages, the production of these bladelets had originally been attributed to Final Magdalenian knappers (Demars 1985). Based on drawings of these bladelets (fig. 22), we were able to observe that the dorsal faces of some of them had invasive transversal removals that could have resulted from the preparation of an inverse truncation on the core. These bladelets could thus have been detached from La Bertonne artifacts. However, to demonstrate this, we had to verify that these transversal scars were not the result of retouch or the vestigial remnants of preparing a crest (fig. 23).

Recent discovery of retouched bladelets obtained from La Bertonne artifacts (fig. 24-28; table 4)

In the La Malignière and Bellevue collections, found in the archaeological deposit of Guéret (Creuse), in the sample of retouched bladelets, we were able to identify five fragments (fig. 24) that were incontestably detached from La Bertonne artifacts. - Invasive transversal scars, visible on the dorsal face of these bladelet fragments, result from preparation prior to the detachment of the bladelet. These scars cannot be mistaken for those of a line of retouch.

- Moreover, the orientation of these scars reveals that they come from the preparation of a truncation and not a crest. The waves are oriented from the edge to the center of the bladelet (fig. 23a), thus the opposite of those visible on scars that result from the creation of a crest (fig. 23b). 
- The recognition of a « positive » surface on the other part of the dorsal face of some bladelets (fig. $24 \mathrm{n}^{\circ} 1-2$ ) permits determination that they were also detached from the ventral face of a blank.

These three technological criteria thus permit us to confirm, and this despite the small number of products known today, that the Badegoulians produced bladelets from La Bertonne artifacts (fig. 25).

By observing these five examples, we can already suggest the intended form of the bladelets. We could observe, based on reading the bladelet scars on the cores (fig. 26), that the knappers obtained bladelets of different sizes. These could be fairly long, others very short and especially extremely thin. Based on the five examples found, bladelets were systematically retouched from the same edge, the one which has the transversal scars. Retouch, generally direct, in four cases out of five, slightly reduces the width of the blank and seems to have been aimed at correcting the delineation of the edge. The opposite edge has no preparation and retains a sharp edge. The armature thus has the form of a backed bladelet.

According to one of the authors (J.P.), such bladelet production has another characteristic. The bladelets obtained from La Bertonne artifacts were removed by pressure flaking (fig. 27-28; table 4). So, the recognition of this removal technique would in the future be essential to identify the use of bladelet production of La Bertonne type.

Synthesis of the use of La Bertonne artifacts as bladelet cores (fig. 29-37)

\section{Knapping technique}

After the analysis of many La Bertonne artifacts, we have determined that the principle of the operatory schema consisted in extracting the first bladelet by pressure flaking, from the ventral face of a generally laminar blank, transversal to its flaking axis from an initial ridge formed by the alignment of distal scars from an inverse truncation. The subsequent ridges were generally exploited, always by pressure flaking, and several bladelets of varying size were obtained from a single production sequence.

\section{Among the consequences of the discovery... (fig. 38)}

To discover that a prehistoric tool is actually a bladelet core is now fairly common for the Upper Paleolithic. The interest is better understood when we note the repercussion that this discovery could have on our knowledge of the period during which these knapping methods were developed. For the technique discussed here, two significant effects are already foreseeable. We now know why La Bertonne artifacts appear to be so similar to Orville artifacts (fig. 38). Having the same function, they can be compared and show that they were two methods governed by the same reduction technique (Chehmana 2009 and doctoral thesis in progress). So, the principle of Orville reduction presented by C. Perlès (1982) and J. Pelegrin (1982) in the 1980 s is also valid for La Bertonne reduction. In both cases, " the knappers intended to remove an [initial] bladelet from a ridge located at the conjunction of an inverse truncation and the ventral face of a blade. " Their similarity is thus not due to a simple convergence in form, but a real technological relationship. We propose the hypothesis that these two methods were part of the same technological lineage, and that one of them is perhaps the technological improvement of the other. From this follows the second repercussion of this discovery. When we say technological relationship, this certainly implies that the groups who invented the techniques lived during relatively close periods in times. Regarding the seriation of the industries, this means that in the future we will connect the Orville industry - placed without great certainty in the middle phase of the Magdalenian - with the La Bertonne industry, attributed to the Badegoulian.

\section{Introduction}

Les préhistoriens qui ont découvert les pièces de la Bertonne (fig.1) ont longtemps pensé qu'il serait délicat de retrouver leur véritable fonction (Daleau 1909 ; Leysalles et Noone 1949). En observant leur morphologie, ils ont présumé qu'il s'agissait d'un outil singulier qui se rapprochait plutôt d'un grattoir mais dont le front était aménagé sur la face inférieure du support. Toutefois, jamais aucune analyse des traces d'utilisation n'est venue confirmer cette première hypothèse. Près d'un siècle après leur découverte, l'énigme n'était donc toujours pas résolue. Deux autres hypothèses fonctionnelles (pièce amincie en vue d'un emmanchement et nucléus à lamelles) ont été depuis évoquées, mais sans que l'une ou l'autre ne soit réellement privilégiée (Lenoir 1987 ; Demars et Laurent 1989 - p. 88).
Nous avons alors décidé de tester ces trois hypothèses en procédant par élimination. C'est à l'aide de modélisations par le dessin ainsi que d'une analyse tracéologique et technologique qu'il a été possible de déterminer si les pièces de la Bertonne, récemment attribuées au Badegoulien (Lenoir 1987, 2000), ont été modifiées :

- en vue d'un emmanchement ?

- pour aménager une partie active ?

- ou encore, pour y extraire des lamelles?

La démonstration que nous proposons ici est le fruit d'une étude entamée depuis 2005 (Chehmana et Bodu 2005) et dont les résultats ont été exposés à l'occasion du colloque sur les «Approches méthodologiques intégrées pour l'étude des outils lithiques » organisé par Laura Longo à Florence en décembre 2007 (Chehmana in pré-actes : 

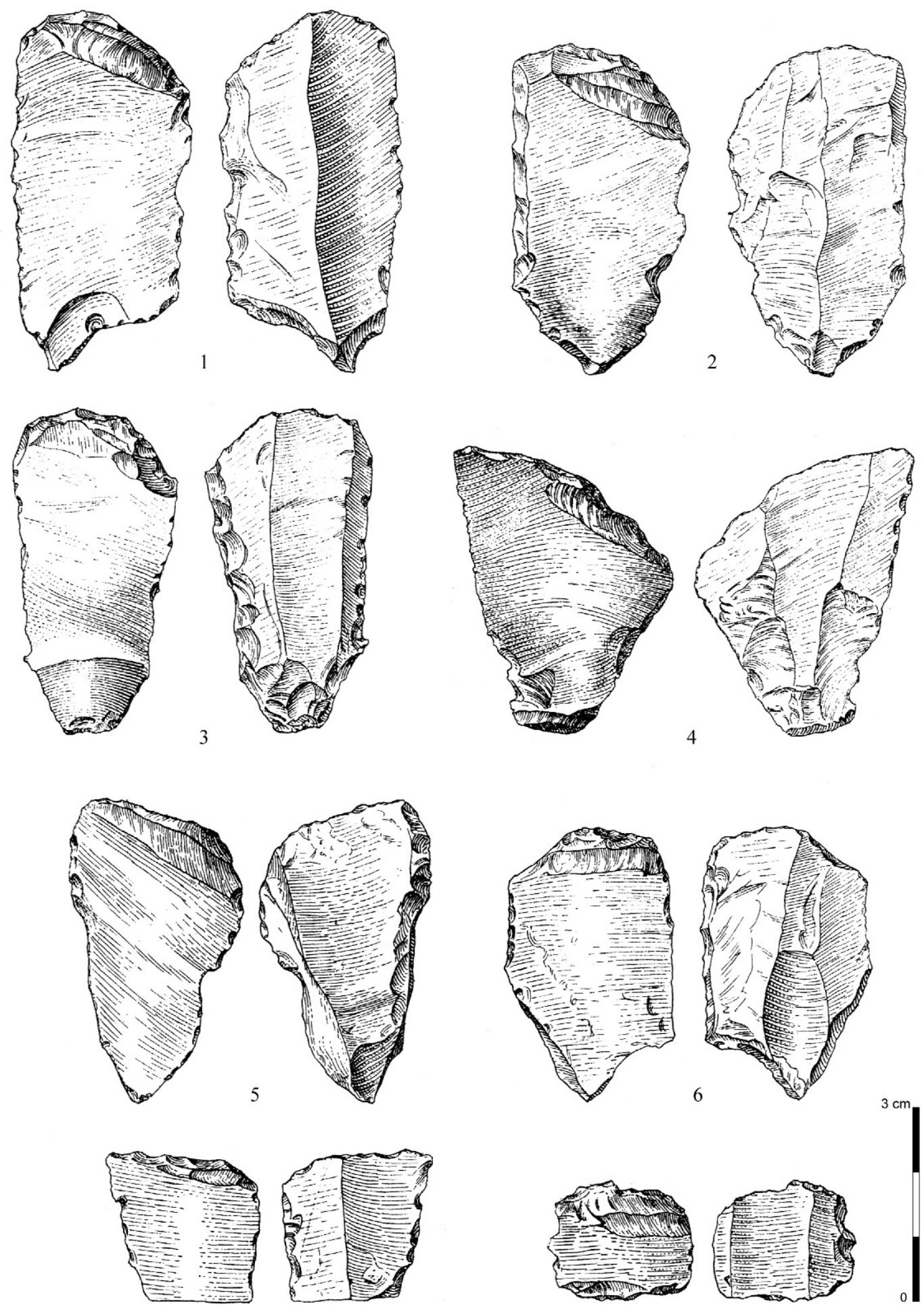

7
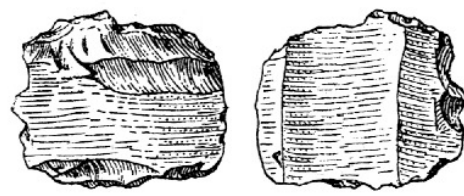

Figure 1 - Les premiers dessins de pièces de la Bertonne (collection Daleau, site de la Bertonne, d'après dessins F. Daleau dans Daleau 1909). $N^{\circ} 1$ (cf. $n^{\circ} 26$, pl. IV) ; $n^{\circ} 2$ (cf. $n^{\circ} 16$, pl. II) ; $n^{\circ} 3$ (cf. $n^{\circ} 18$, fig. 5) ; $n^{\circ} 4$ (cf. $n^{\circ} 15$, pl. II) ; $n^{\circ} 5$ (cf. $n^{\circ} 31, p l . V$ ) ; $n^{\circ} 6$ (cf. $n^{\circ} 32$, pl. V) ; $n^{\circ} 7$ (cf. $n^{\circ} 34$, pl. V) ; $n^{\circ} 8$ (cf. $n^{\circ} 29$, pl. V).

Figure 1 - The first drawings of La Bertonne artifacts (Daleau collection, site of La Bertonne, after drawings by F. Daleau in Daleau 1909). $N^{\circ} 1$ (cf. $\left.n^{\circ} 26, p l . ~ I V\right) ; n^{\circ} 2$ (cf. $n^{\circ} 16$, pl. II); $n^{\circ} 3$ (cf. $n^{\circ} 18$, fig. 5); $n^{\circ} 4$ (cf. $\left.n^{\circ} 15, p l . ~ I l\right) ; n^{\circ} 5$ (cf. $\left.n^{\circ} 31, p l . ~ V\right) ; n^{\circ} 6$ (cf. $\left.n^{\circ} 32, p l . ~ V\right) ;$ $n^{\circ} 7$ (cf. $n^{\circ} 34$, pl. V); $n^{\circ} 8$ (cf. $n^{\circ} 29$, pl. V). 
« Identification of a technological innovation in Badegoulian lithic industries in the northern half of France : the example of bladelet production of "Orville » and "Bertonne » types "). Ces résultats ont été publiés sous une forme abrégée dans les actes du colloque parus dans la revue Human Evolution (Chehmana 2009).

La comparaison entre les pièces de la Bertonne et celles d'Orville et des méthodes de taille dont elles résultent sera présentée en détail dans une prochaine publication (Chehmana, sous presse).

\section{Historique de la découverte des pièces de la Bertonne et de leurs dénominations successives}

Les premières pièces de la Bertonne ont été découvertes lors d'un ramassage de surface, par F. Daleau à la fin du XIX siècle au lieu-dit la Bertonne ou la Rousse, situé sur la commune de Peujard en Gironde (Daleau 1909). II en fit part à la communauté des préhistoriens 15 ans plus tard en publiant les premiers dessins (fig. 1) dans le bulletin de la Société archéologique de Bordeaux (ibid.). II les dénommait « compresseurs-retouchoirs à retaille inverse transversale " (Daleau 1909 - p. 36). II employa également des expressions plus neutres comme celles de " silex à retouches anormales ", c'est-à-dire inverses, selon la dénomination de l'époque (Daleau 1909, 1911). Près de 40 ans plus tard, G. Leysalles et H.V.V. Noone (1949) en découvrirent de nouveaux exemplaires au Pech de SaintSourd, situé sur la commune des Eyzies-de-Tayac, en Dordogne. Ils proposèrent une autre dénomination puisqu'à cette époque, ces derniers n'avaient pas encore été apparentés aux pièces découvertes par F. Daleau. Ils suggérèrent le terme de " grattoir de Saint-Sourd ", introduisant ainsi, dans la dénomination même, une autre interprétation fonctionnelle ${ }^{5}$. Par la suite, dans les années soixante-dix, M. Lenoir entreprit l'étude de l'ensemble de la série provenant du site de la Bertonne (Lenoir 1976). II décrivit précisément ces pièces "à retouches anormales " et proposa finalement de leur donner une appellation plus « classique » en Préhistoire : il souhaitait qu'elles portent le nom du site sur lequel elles avaient été trouvées pour la première fois. C'est donc depuis ses travaux que nous les désignons : "pièces de la Bertonne » (ibid.).

\section{Description des pièces de la Bertonne}

G. Leysalles et H.V.V. Noone (1949) seront les premiers à proposer une description relativement succincte de ces pièces. Selon les auteurs, les « grattoirs de Saint-Sourd présentent, à l'une de leurs extrémités et sur leur face inférieure, une série de longues cannelures transversales et plus ou moins parallèles résultant de l'enlèvement de lamelles par une pression ou percussion quelconque exercée latéralement sur la face supérieure gauche de la lame " (Leysalles et Noone 1949 - p. 251). À cette définition quasiment complète, $M$. Lenoir apporta un élément supplémentaire : la présence, sur de nombreux exemplaires, d'une troncature inverse systématiquement recoupée par ces négatifs d'enlèvements lamellaires (Lenoir 1987 p. 167). Ainsi, il évoqua pour la première fois l'ordre d'exécution des opérations de taille. Sa définition sera reprise et synthétisée dans l'ouvrage récapitulatif des différents types d'outils du Paléolithique supérieur en Europe où P.-Y. Demars et P. Laurent les définissent comme des " outils sur lame ou sur éclat présentant sur la face inférieure une série de retouches lamellaires, " plan », transversales à l'axe de la pièce, partant du bord situé à gauche d'une troncature inverse façonnée antérieurement à cette série d'enlèvements » (Demars et Laurent 1989 - p. 88).

Pour résumer, quatre critères (fig. 2) doivent être réunis pour qualifier une pièce de la Bertonne:

- premièrement, le support est généralement une lame (plutôt mince d'après nos propres observations) ;

- deuxièmement, les pièces de la Bertonne présentent une troncature inverse ;

- troisièmement, cette troncature inverse est recoupée par un ou plusieurs enlèvements lamellaires, de plus en plus plans ;

- quatrièmement, les enlèvements lamellaires sont extraits quasi-systématiquement depuis le bord situé à droite de la troncature inverse lorsqu'elle est orientée vers le haut (autrement dit : du bord gauche du support si la troncature est distale, de son bord droit si la troncature est proximale).

\section{Localisation géographique des assemblages comportant des pièces de la Bertonne}

Lors de ses recherches, M. Lenoir entreprit une recension de l'ensemble des séries comportant des pièces de la Bertonne (Lenoir 1987). Jusqu'au début des années soixante-dix, elles ont été essentiellement signalées dans le sud-ouest de la France, dans les départements de la Gironde et de la Dordogne (fig. 3). Entre les années soixante-dix et quatre-vingt, d'autres furent recensées, d'abord dans les départements limitrophes, comme les Landes, la Corrèze et la Charente-Maritime, mais également un peu plus au nord, dans le département de la Creuse. Une dernière série fut localisée récemment dans une région plus septentrionale encore, le long de la vallée de la Loire (Valentin 1995 - p. 65). Elle provient d'un ramassage de surface effectué par l'un d'entre nous (P. B.), à l'est de la ville de Gien (Loiret), au lieu dit du Trocadéro. Enfin, F. Daleau signale l'existence de pièces similaires dans une série qui provient du lieu-dit la Caboche, à Saint-Pierre-du-

(5) F. Daleau avait également évoqué l'idée que les pièces qu'il venait de découvrir sur le site de la Bertonne s'apparentaient à des «pseudo-grattoirs » (Daleau 1909 - p. 42). 


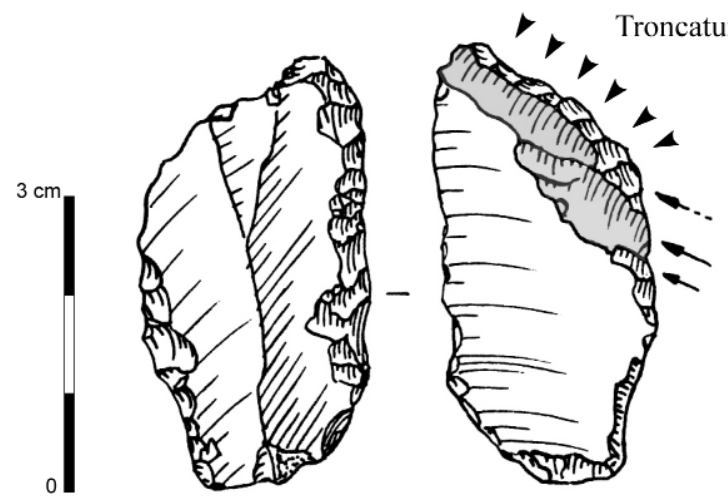

Support laminaire mince
La troncature inverse est recoupée

par un enlèvement lamellaire ;

il est extrait quasi-systématiquement

depuis le bord situé à droite

de la troncature inverse

lorsqu'elle est orientée vers le haut
Figure 2 - Premiers critères essentiels pour identifier une pièce de la Bertonne (d'après dessins M. Lenoir dans Lenoir 1976 p. 45, fig. 1).

Figure 2 - Initial essential criteria to identify a La Bertonne artifact (after drawings by M. Lenoir in Lenoir 1976, p. 45, fig. 1).

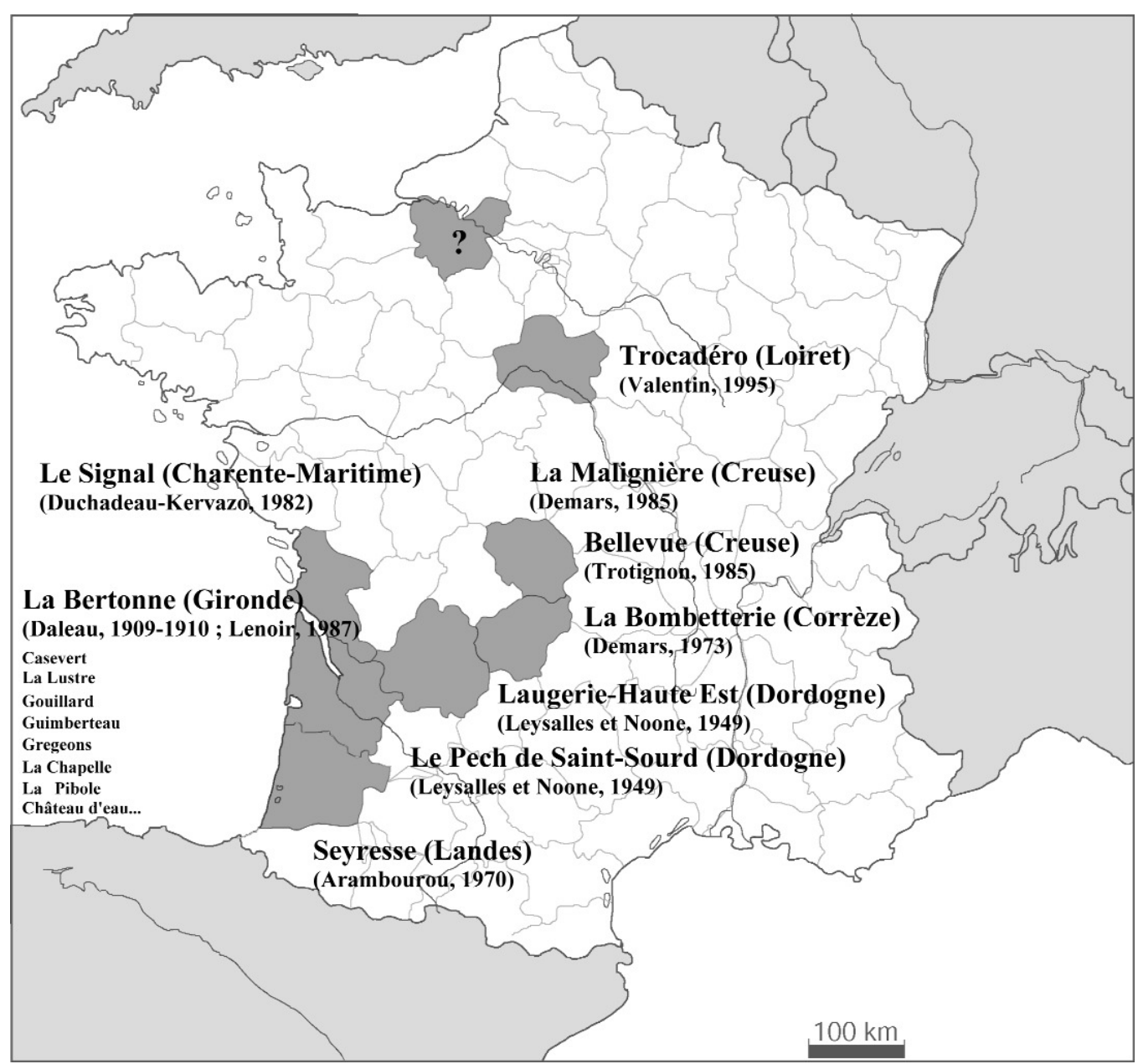

Figure 3 - Localisation par département des principaux sites dans lesquels ont été retrouvées des pièces de la Bertonne. L'essentiel des références a été publié par M. Lenoir en 1987 (DAO L. Chehmana).

Figure 3 - Location by department of the main sites in which La Bertonne artifacts have been found. Most references were published by M. Lenoir in 1987 (DAO, L. Chehmana). 
Vauvray (Eure), en Haute-Normandie (1910 - note p. 276). Nous avons tenté de retrouver cette collection, malheureusement, elle fut détruite durant la Seconde Guerre mondiale. Quoi qu'il en soit, depuis les premières découvertes de pièces de la Bertonne, l'aire de répartition connue pour ces artefacts s'est finalement étendue du bassin de l'Adour jusqu'à la vallée de la Loire.

\section{À quel moment apparaissent les pièces de la Bertonne?}

La position chronologique des assemblages à pièces de la Bertonne est relativement imprécise. Essentiellement découverts sur des sites de plein air, les assemblages qui en comportent le plus n'ont pu faire l'objet de datation. Quelques pièces de la Bertonne (fig. 4) ont toutefois été signalées en stratigraphie, à Laugerie-Haute, dans les couches I' et I" des fouilles D. Peyrony attribuées à l'époque au Magdalénien I (Leysalles et Noone 1949 - p. 250 et 251), mais également dans les couches 4 et 10 des fouilles F. Bordes (fig. 5) (Hemingway 1980 - p. 324 et 335 ; Demars et Laurent 1989 - p. 88 et 89 ; Lenoir 1987). Anciennement rattachés à l'Aurignacien (Leysalles et Noone 1949 ; D. Peyrony 1949 - p. 30), les assemblages à pièces de la Bertonne ont donc, depuis ces dernières découvertes, été réattribués au Badegoulien (Lenoir 1987, 2000) $)^{6}$. En l'état actuel des connaissances, il est toutefois impossible de préciser davantage le moment de leur apparition ainsi que d'évaluer le temps durant lequel les Badegouliens ont conçu ces pièces de la Bertonne.

Notons qu'une pièce assimilée aux Bertonne (fig. 6) fut également signalée par $\mathrm{M}$. Lenoir dans une couche solutréenne de Laugerie-Haute Est (Lenoir 1987 - p. 169). L'hypothèse de leur apparition durant le Solutréen ne nous paraît cependant pas envisageable car, d'après le dessin (Smith 1966 - p. 137, fig. 29 n 6), cette pièce ne présente pas toutes les caractéristiques d'une «Bertonne » :

- la troncature inverse est absente,

- le support n'est pas laminaire.

L'interprétation de cette pièce est donc discutable puisqu'elle pourrait également s'apparenter à un burin ou à un simple nucléus à lamelles sur tranche d'éclat.

Retenons qu'actuellement, l'attribution des Bertonne au Badegoulien tient surtout au fait que ces artefacts sont régulièrement associés à des raclettes au sein des assemblages (Lenoir 1987). Les " raclettes " sont des outils sur éclat relativement mince qui présentent une retouche abrupte et dont l'appellation date des années 1930
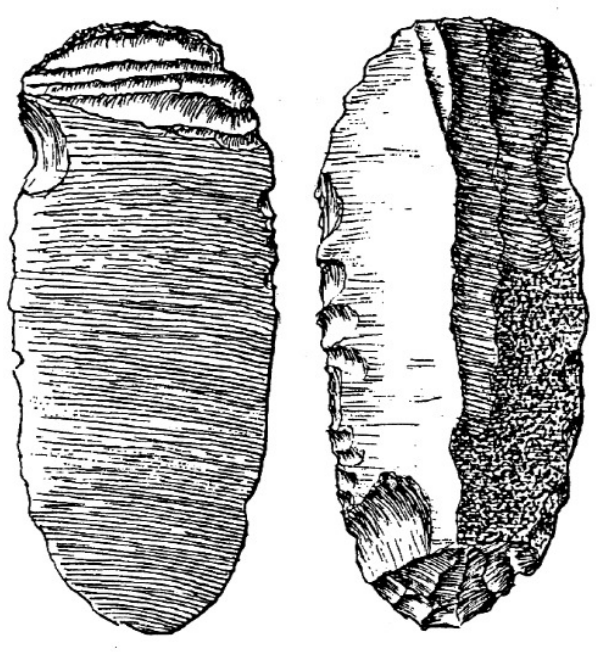

1

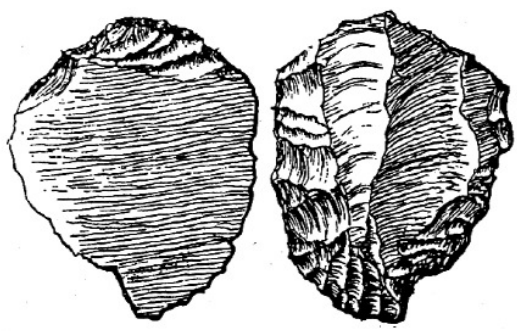

3

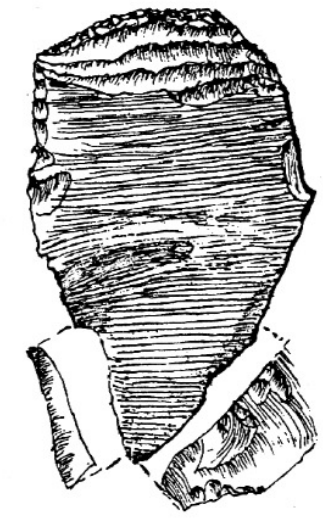

2

Figure 4 - Trois exemplaires de pièces de type "Bertonne » provenant des fouilles réalisées à Laugerie-Haute par D. Peyrony, couches I' et I" (d'après Leysalles et Noone 1949, p. 250, fig. 2).

Figure 4 - Three examples of artifacts of « $\mathrm{La}$ Bertonne » type from excavations at Laugerie-Haute by D. Peyrony, layers I' and I" (after Leysalles \& Noone 1949, p. 250, fig. 2).

(6) Anciennement qualifié de Magdalénien I ou ancien (Peyrony D. et E. 1938) ou encore de Magdalénien 0 et I (Bordes 1958). 


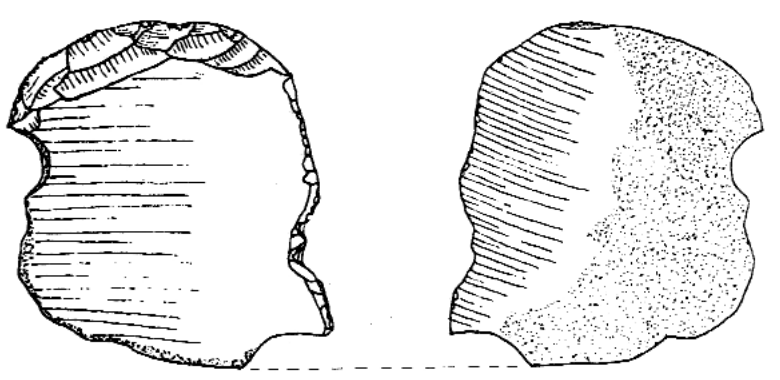

1
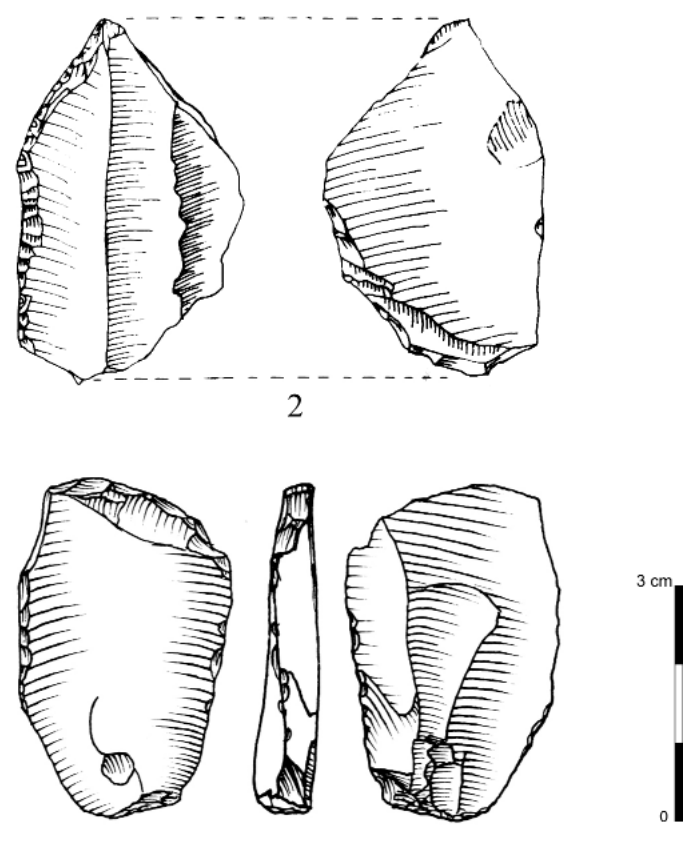

3

Figure 5 - Trois exemplaires de pièces de type "Bertonne » provenant des fouilles réalisées à Laugerie-Haute Est par $F$. Bordes ( $n^{\circ} 1$ : niveau 4 et $n^{\circ} 2$ : niveau 10 d'après Hemingway 1980 , p. 324 , fig. I.10 et p. 335 , fig. I.12; $n^{\circ} 3$ : niveau 10 d'après Demars et Laurent 1989, p. 89, fig. 30).

Figure 5 - Three examples of "La Bertonne » type from excavations at Laugerie-Haute by F. Bordes ( $n^{\circ}$ 1: layer 4 and $n^{\circ}$ 2: layer 10, after Hemingway 1980, p. 324, fig. I.10 and p. 335, fig. I.12; $n^{\circ}$ 3: niveau 10, after Demars \& Laurent 1989, p. 89, fig. 30 ).

(Cheynier 1930). Elles sont considérées comme l'un des fossiles directeurs de la seconde phase du Badegoulien (Vignard 1965 ; Bosselin et Djindjan 1988). Les mesures 14C effectuées sur des fragments organiques prélevés au sein des assemblages comportant des raclettes sont essentiellement comprises entre 18500 et 16500 BP (tabl.1). D'après les recensions de M. Lenoir, la moitié des collections à pièces de la Bertonne en comporteraient

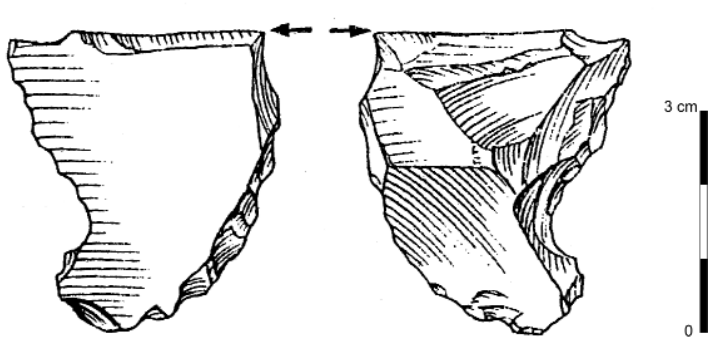

Figure 6 - Pièce associée aux mobiliers solutréens de LaugerieHaute Est, couche 21, (fouilles F. Bordes, d'après Smith 1966 p. 137 fig. 29).

Figure 6 - Artifact associated with Solutrean artifacts at Laugerie-Haute Est, layer 21, (excavations by F. Bordes, after Smith 1966, p. 137, fig. 29).

(Lenoir 1987). Nous avons pu confirmer leur présence (fig. 7) ainsi que celle des nucléus à partir desquels les supports ont été obtenus dans les séries provenant des sites de la Malignière et de Bellevue (Creuse ; Demars 1985 ; Trotignon 1985). II faut toutefois préciser que le mobilier provient essentiellement de ramassages de surface (ce qui est également le cas pour l'essentiel des autres collections à Bertonne connues). II n'a donc encore jamais été possible de retrouver un nombre assez significatif de pièces de la Bertonne (plus d'une dizaine) associées à des raclettes au sein d'un même niveau stratigraphique? ${ }^{7}$.

Toutefois, lorsque les assemblages à pièces de la Bertonne ne livrent pas de « véritables » raclettes, des outils similaires y sont fréquemment attestés (Lenoir 1976 - p. 43 ; Vialou 2004 - p. 824). Nous avons illustré ceux qui ont été recueillis sur le site éponyme de la Bertonne (fig. 8). Les éclats ne portent certes pas les mêmes types de retouche, mais ont une morphologie très proche de celle des supports de raclette (fig. $8 \mathrm{n}^{\text {os }} 1$ et 2). Ils sont également produits de manière sensiblement identique. Issus dans ces deux cas de nucléus sur éclat (fig. $8 \mathrm{n}^{\circ \mathrm{s}} 3$ et 4 ), les supports sont obtenus à partir de deux surfaces relativement planes, par des séries d'enlèvements légèrement juxtaposées, parfois même quasi-superposées. L'agencement de ces enlèvements permet d'obtenir des éclats relativement minces dont l'épaisseur du bord est régulière sur tout le pourtour et inférieure ou égale à $4 \mathrm{~mm}$, excepté à l'emplacement du talon. La morphologie de ce dernier qui s'apparente à une « aile d'oiseau » (fig. $8 \mathrm{n}^{\circ}$ 2) est l'indice évident de la mise en œuvre de cette méthode de débitage particulière, comme cela a déjà été signalé pour les supports de raclette (Morala 1993). À l'heure actuelle, une telle production n'est attestée dans le Paléolithique supérieur d'Europe occidentale qu'entre 19000 et 16000 BP, au sein d'assemblages attribués au Badegoulien et au Magdalénien inférieur (Lenoir 1983 ; Morala 1993 ; Cretin 1996 ; Fourloubey 1996 ; Bracco 1992 ; Cazals 2000 ; Bracco et al. 2003 ; Cazals 2005 ; Langlais 2007).

(7) Excepté peut-être sur le site de Seyresse (Merlet 2005 - p. 105) : "Outre les pièces de la Bertonne, l'outillage comprend 6 raclettes sur petits éclats minces, [...] ». Cependant, la présence de ces raclettes n'est pas vérifiable puisqu'elles n'ont toujours pas été illustrées. 


\begin{tabular}{|c|c|c|c|}
\hline Département & Site & Date (réf.) & Référence bibliographique \\
\hline Nièvre & Oisy & $5920 \pm 40$ BP (Béta-172041) & Bodu et al. 2005 p. 94 \\
\hline Nièvre & Oisy & $7550 \pm 50$ BP (Béta-172040) & $"$ \\
\hline Nièvre & Oisy & $8930 \pm 40$ BP (Béta-172039) & $"$ \\
\hline Nièvre & Oisy & $9030 \pm 40$ BP (Béta-160916) & $"$ \\
\hline Nièvre & Oisy & $12500 \pm 120$ BP (Béta-160913) & $"$ \\
\hline Nièvre & Oisy & $15900 \pm 100$ BP $($ Ly-4621)* & Bodu et al. à paraître \\
\hline Nièvre & Oisy & $16020 \pm 150$ BP (Béta-160915)* & Bodu et al. 2005 p. 94 \\
\hline Nièvre & Oisy & $17820 \pm 120 \mathrm{BP}(\mathrm{Ly}-4622) *$ & Bodu et al. à paraître \\
\hline Indre & Fritsch (c3a) & $17130 \pm 550$ BP (Ly-1121) & Evin et al. 1978 p. 50, Trotignon et al. 1984 p. 96 \\
\hline Indre & Fritsch (c4) & $16530 \pm 350$ BP $($ Ly-1122) & 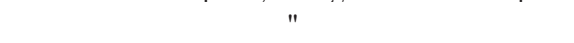 \\
\hline Indre & Fritsch $(c 5 b)$ & $14960 \pm 380$ BP $($ Ly-1001) & " \\
\hline Indre & Fritsch (c5b) & $17280 \pm 350$ BP $($ Ly-1123) & $"$ \\
\hline Indre & Fritsch (c6) & $17960 \pm 350 \mathrm{BP}(\mathrm{Ly}-1124)$ & $"$ \\
\hline Vienne & Taillis des Coteaux (ens. V) & $18140 \pm 85$ BP $($ Ly-2639)* & Primault et al. 2007 p. 11 \\
\hline Haute-Loire & Blot (n15) & $18000 \pm 80 \mathrm{BP}(\mathrm{Ly}-1337 / \mathrm{GrA}-17337) *$ & Daugas et Delporte en préparation \\
\hline Haute-Loire & Cottiers (c2) & $18550 \pm 550 \mathrm{BP}(\mathrm{Ly}-719)$ & Evin et al. 1975 p. 27 \\
\hline Haute-Loire & Rond du Barry (cF2) & $17100 \pm 450$ BP (Gif-3038) & De Bayle des Hermes 1979 p. 602 \\
\hline $\begin{array}{l}\text { Dordogne } \\
\text { Dordogne }\end{array}$ & $\begin{array}{l}\text { Casserole (NA4) } \\
\text { Casserole (NA5) }\end{array}$ & $\begin{array}{l}16960 \pm 60 \text { BP (Gif-LSM 9193) } \\
17120 \pm 60 \text { BP (Gif-LSM 9191) }\end{array}$ & $\begin{array}{c}\text { Detrain et al. } 1994 \text { Annexe } \\
\text { " }\end{array}$ \\
\hline Dordogne & Laugerie-Haute Est (Magd II) & $17040 \pm 440 \mathrm{BP}(\mathrm{Ly}-973)$ & Evin et al. 1976 p. 80 \\
\hline $\begin{array}{l}\text { Dordogne } \\
\text { Dordogne }\end{array}$ & $\begin{array}{l}\text { Jamblancs (abri ouest c2) } \\
\text { Jamblancs (abri ouest c2) }\end{array}$ & $\begin{array}{c}17770 \pm 260 \text { BP (Ly-4589) } \\
16490 \pm 130 \text { BP (Gif) }\end{array}$ & $\begin{array}{l}\text { Cleyet-Merle } 1992 \text { p. } 232 \\
\text { Cleyet-Merle } 1992 \text { p. } 233\end{array}$ \\
\hline Lot & Cuzoul de Vers (c5) & $15980 \pm 150$ BP (Gif-6638) & Clottes et Giraud 1989 p. 90 \\
\hline Lot & Cuzoul de Vers (c13) & $16800 \pm 170$ BP (Gif-6371) & $"$ \\
\hline Lot & Cuzoul de Vers (c20) & $17050 \pm 170 \mathrm{BP}($ Gif-6797) & Clottes et Giraud 1989 p. 89 \\
\hline Lot & Piage (cC-E mélange Sol+Badeg) & $18900 \pm 250 \mathrm{BP}($ Gif-5026) & Champagne et Espitalie 1981 p. 202 \\
\hline Lot & Pégourié (c8A2) & $16890 \pm 300 \mathrm{BP}(\mathrm{Ly}-5257)$ & Séronie-Vivien 1995 p. 46 \\
\hline Lot & Pégourié (c8) & $17320 \pm 420$ BP (Ly-1834) & $"$ \\
\hline Lot & Pégourié (c8B) & $17490 \pm 520 \mathrm{BP}($ Ly-1394) & $"$ \\
\hline Lot & Pégourié (c9A) & $17420 \pm 390 \mathrm{BP}(\mathrm{Ly}-1836)$ & $"$ \\
\hline Lot & Peyrugues (c9) & $18100 \pm 140 \mathrm{BP}($ Gif 7996) & Chalard 1993 p. 19 \\
\hline Aude & Lassac & $16580 \pm 80$ BP (Gra 18788/Ly-1548)* & Sacchi 2003 p. 160 \\
\hline Aude & Lassac & $16750 \pm 250$ BP (Gif-2981) & Sacchi 1976 p. 1180 \\
\hline
\end{tabular}

Tableau 1 - Datations au radiocarbone effectuées sur les couches archéologiques ayant livré des raclettes (note * : datation récente réalisée avec la méthode $A M S$ ).

Table 1 - Radiocarbon dates from archaeological layers with raclettes (note*: recent date made by AMS).

\section{Représentativité des assemblages archéologiques recensés et examinés}

Sur la vingtaine d'assemblages connus, dont une dizaine avec raclettes ou « éclats apparentés », six ensembles seulement livrent plus d'une quinzaine de pièces de la Bertonne (tabl. 2). Celui provenant du Pech de Saint-Sourd doit malheureusement être écarté de cette liste puisqu'il reste introuvable. Les dernières informations divulguées par H. L. Movius à son sujet (in Bricker 1995 - p. 262), font état d'« une petite série représentative, comprenant plusieurs " grattoirs de Saint-Sourd ", conservée par G. Leyssales à la villa du Cro-Magnon aux Eyzies. Le reste a été emporté en Angleterre par H.V.V. Noone et l'on ignore ce qu'est devenue la collection ». Cette série comportait les uniques éléments osseux que l'on aurait pu prélever pour datation (Leysalles et Noone 1949 - note p. 247).

Sur les cinq séries encore disponibles, qui comportent plus d'une quinzaine de pièces de la Bertonne, nous avons étudié de façon exhaustive celle encore inédite du Trocadéro, qui livre soixante sept exemplaires. Notre argumentation a été essentiellement fondée sur leur analyse, parfois complétée, au besoin, par des observations issues de l'examen des pièces provenant du site éponyme de la Bertonne. Les collections de la Malignière et de Bellevue ont également été prises en compte car nous y avons découvert des lamelles retouchées dont les supports ont été incontestablement obtenus à partir des pièces de la Bertonne (cf. infra fig. 24). Quant aux séries du Signal et de Seyresse, nous ne les avons pas examinées ${ }^{8}$. II n'était cependant pas

(8) La série de Seyresse a fait l'objet d'une étude récente (Ducasse et Langlais 2008) dont les résultats convergent avec ceux que nous avions exposés à l'occasion du colloque de Florence en décembre 2007 et que nous détaillons ici plus amplement. 

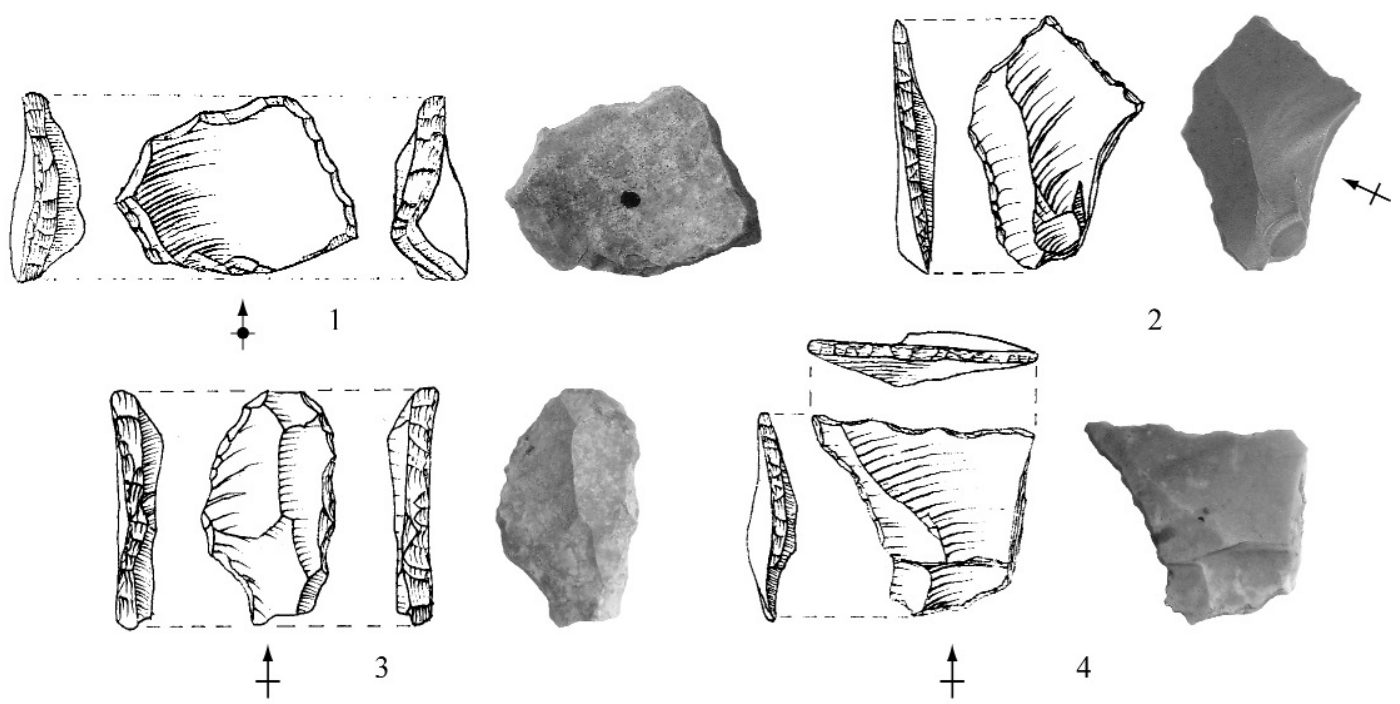

2
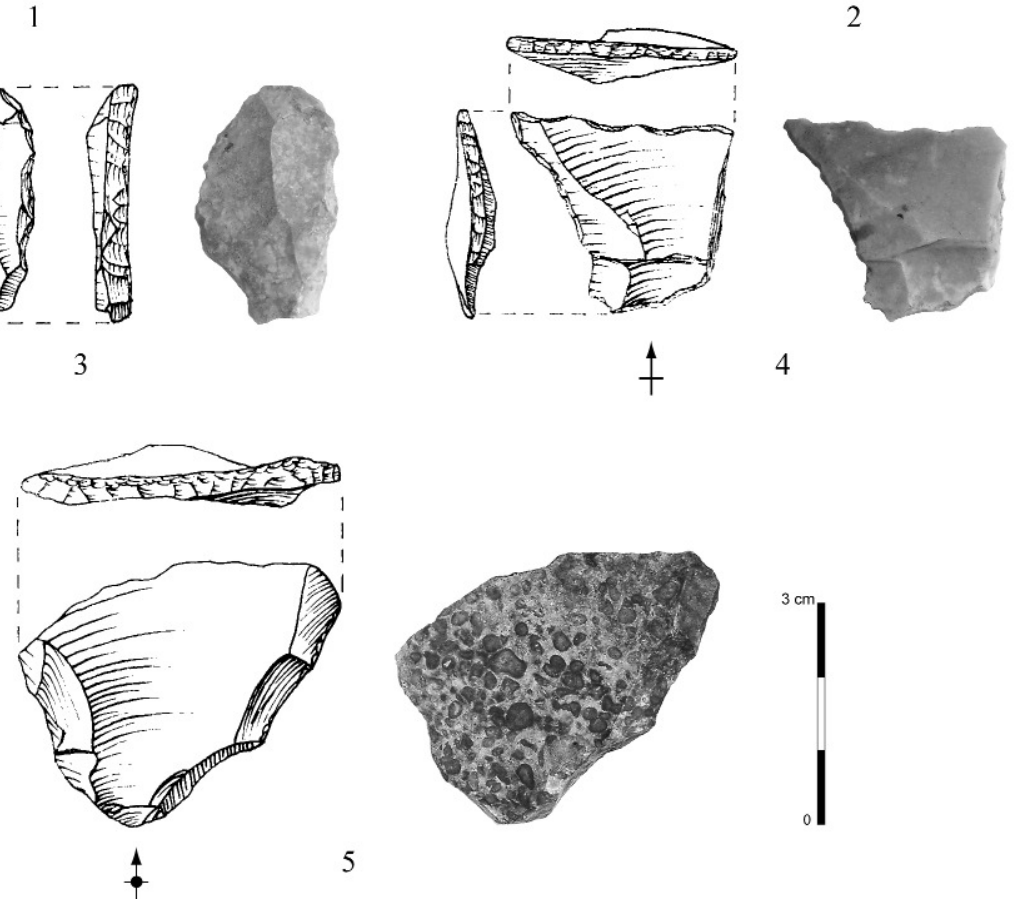

Figure 7 - Quelques exemplaires de raclettes associés aux pièces de la Bertonne sur le site de la Malignière (collection Lasnier, d'après dessins P.-Y. Demars dans Demars 1985 fig. 6, clichés L. Chehmana).

Figure 7 - Examples of raclettes associated with La Bertonne artifacts at the site of La Malignière (Lasnier collection, after drawings by P.-Y. Demars in Demars 1985, fig. 6, photo by L. Chehmana).

nécessaire d'analyser l'ensemble de ces assemblages pour répondre à notre problématique.

Nous en profitons pour signaler la découverte récente d'une pièce de la Bertonne isolée (fig. 9), retrouvée en surface $^{9}$, devant le site de la Rochette (Saint-Léon-surVézère, Dordogne), où aucune occupation badegoulienne n'avait été recensée jusqu'à présent (Movius in Bricker 1995 - p. 292).

\section{Les différentes hypothèses fonctionnelles évoquées pour les pièces de la Bertonne}

Les pièces de la Bertonne ont d'abord été envisagées comme des instruments qui auraient été utilisés pour la fabrication d'un outillage commun. C'est ce que F. Daleau
(1909) sous-entendait lorsqu'il employait les termes de " retouchoir " ou " compresseur ". C'est à partir des recherches menées par G. Leysalles et H.V.V. Noone (1949) qu'elles ont été rapprochées d'outils du fond commun. D'après ces auteurs, la morphologie de la partie active s'apparentait plutôt à celle des fronts de grattoir. Jusqu'à aujourd'hui, les analyses ou descriptions supplémentaires n'ont jamais réellement contredit ce rapprochement (Sonneville-Bordes 1960 - p. 71 ; Brézillon 1968 - p. 238 ; Rigaud 1986 - p. 241 ; Movius in Bricker 1995 - p. 262 ; Vialou 2004 - p. 824). M. Lenoir a décrit précisément la procédure de fabrication et en conclut également qu'il pourrait s'agir d'un grattoir inverse (Lenoir 1976, 1987). Il est par ailleurs le seul à avoir évoqué l'idée que les modifications observées sur les pièces de la Bertonne pourraient résulter d'un « procédé d'amincissement » (Lenoir 1976 - p. 46).

(9) Nous avons été informés de l'existence de cette pièce par J.L. Piel Desruisseaux (via l'un d'entre nous, J.P.). Nous le remercions de nous avoir autorisés à la dessiner et la photographier. 

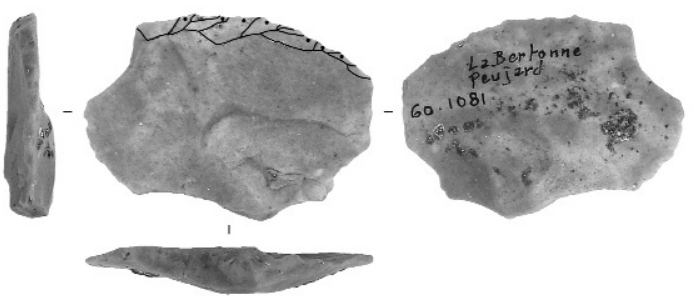

1
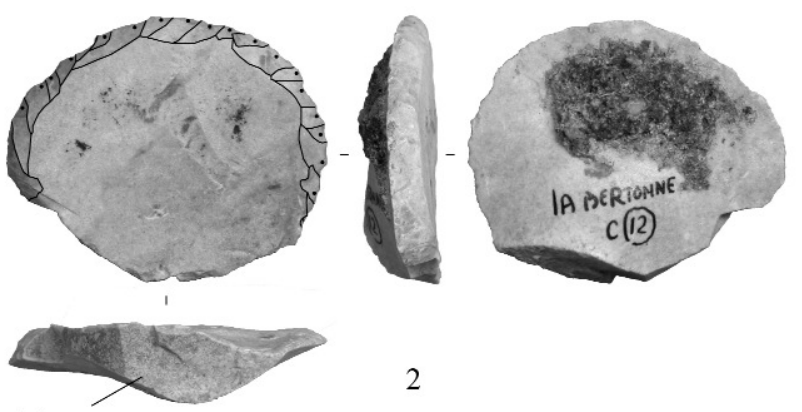

2

Talon en "aile d'oiseau"
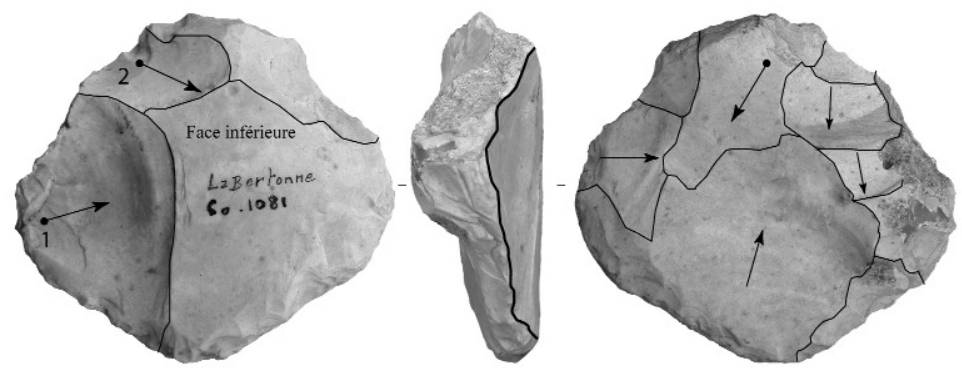

3
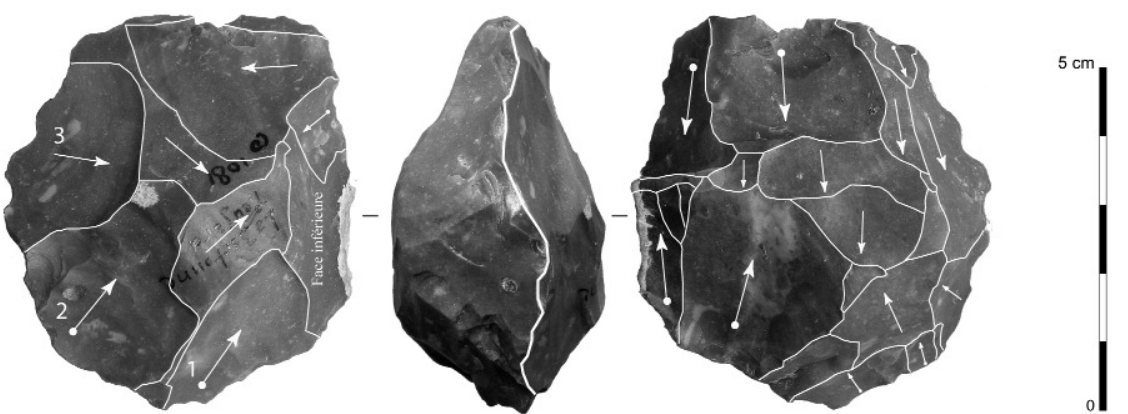

Figure 8 - Photographie de deux outils confectionnés sur des éclats minces ( $n^{\text {os }} 1$ et 2) et de deux nucléus ( $n^{\circ s} 3$ et 4) à partir desquels les mêmes types de support ont été obtenus (collection Daleau, site de la Bertonne, clichés L. Chehmana).

Figure 8 - Photo of two tools made on thin flakes ( $n^{\circ s} 1$ and 2) and cores ( $n^{\circ s} 3$ and 4 ) from which the same same type of blank was produced (Daleau collection, site of La Bertonne, photos by L. Chehmana).

4

Cet amincissement aurait-il été effectué pour faciliter l'insertion des lames dans un manche ? Afin d'estimer si les Badegouliens ont pu véritablement concevoir cette zone comme une partie destinée à être emmanchée, nous avons procédé à des modélisations par le dessin. Nous avons par ailleurs cherché à savoir si ces aménagements n'avaient pas plutôt été réalisés dans le but d'installer aux extrémités des lames une partie active. Pour cela, il a fallu déterminer si ces extrémités portaient ou non des traces d'utilisation.
Enfin, plus récemment, les pièces de la Bertonne ont été comparées aux pièces d'Orville (Perlès 1977, 19811982 note p. 131 ; Lenoir 1983 - p. 269, 1987 ; Demars et Laurent 1989 - p. 88). Or, ces dernières (fig. 10) ne sont pas considérées comme des outils puisqu'il a été démontré qu'elles étaient vraisemblablement des nucléus à lamelles (Perlès 1982 ; Pelegrin 1982). Étant donné que les pièces de la Bertonne et d'Orville ont en commun une série d'opérations techniques (aménagement d'une troncature inverse 


\begin{tabular}{|c|c|c|c|c|}
\hline Département & Site & Collection & \begin{tabular}{|l|} 
Nombre de pièce de la Bertonne \\
\end{tabular} & Référence bibliographique \\
\hline \multirow[t]{12}{*}{ Gironde } & La Bertonne & Daleau et Fredon & 157 & (Daleau 1909; Lenoir 1976) \\
\hline & Casevert & - & 6 & (Lenoir 1987) \\
\hline & La Chapelle & Boireau & 4 & (Lenoir 1987) \\
\hline & La Pibole & Boireau & 3 & (Lenoir 1987) \\
\hline & Gouillard & - & 3 & (Lenoir 1987) \\
\hline & Croûte Charlus & Daleau & 1 & (Lenoir 1983) \\
\hline & La Lustre & - & 1 & (Lenoir 1987) \\
\hline & Coudet & Daleau & 1 & (Lenoir 1987) \\
\hline & Bel-Air & Maziaud & 1 & (Lenoir 1987) \\
\hline & Guimberteau & Cousté & 1 & (Lenoir 1987) \\
\hline & Gregeons & Obry & 1 & (Lenoir 1987) \\
\hline & Château d'eau & Boireau & 1 & (Lenoir 1987) \\
\hline \multirow[t]{5}{*}{ Dordogne } & Pech de Saint-Sourd & Leysalles & 35 & (Leysalles et Noone 1949 p. 251) \\
\hline & Laugerie-Haute & Peyrony et Bordes & 6 & (Leysalles et Noone 1949 p. $250-251$; Hemigway 1980 p. 324 et 335 ) \\
\hline & Jamblancs & Peyrony & 1 & (Cleyet-Merle 1992 p. 232) \\
\hline & Badegoule & Cheynier & 1 & (Cretin 2000 pl. LXXXXIX p. 145) \\
\hline & La Rochette & Piel Desruisseaux & 1 & Inédit \\
\hline \multirow[t]{2}{*}{ Les Landes } & Seyresse & Arambourou & 16 & (Arambourou 1970 p. 4 ; Lenoir 1987 p. 169 ; Merlet 2005 p. 105 ; Ducasse et Langlais 2008 p. 69) \\
\hline & Le Réservoir & Merlet & 2 & (Merlet 2005 p. 107) \\
\hline Corrèze & Bombetterie & Cheynier & 5 & (Demars 1973 p. 312) \\
\hline Charente-Maritime & Le Signal & Rocher & 19 & (Duchadeau-Kervazo 1982 p. 672) \\
\hline \multirow[t]{2}{*}{ Creuse } & La Malignière & Lasnier & 25 & (Demars 1985 p. 29 et 31) \\
\hline & Bellevue & Lasnier & 9 & (Trotignon 1985 p. 42) \\
\hline Loiret & Trocadéro & Bazin & 67 & (Valentin 1995 p. 65) \\
\hline Pyrénées-Atlantiques & Azkonzilo & Chauchat & 2 & (Chauchat 1999 p. 135 ; Dachary 2002 p. 48) \\
\hline
\end{tabular}

Tableau 2 - Décompte des pièces de la Bertonne par gisement (classé par département et dans l'ordre chronologique des découvertes).

Table 2 - Frequencies of La Bertonne artifacts by site (sorted by department and in chronological order of the discoveries).
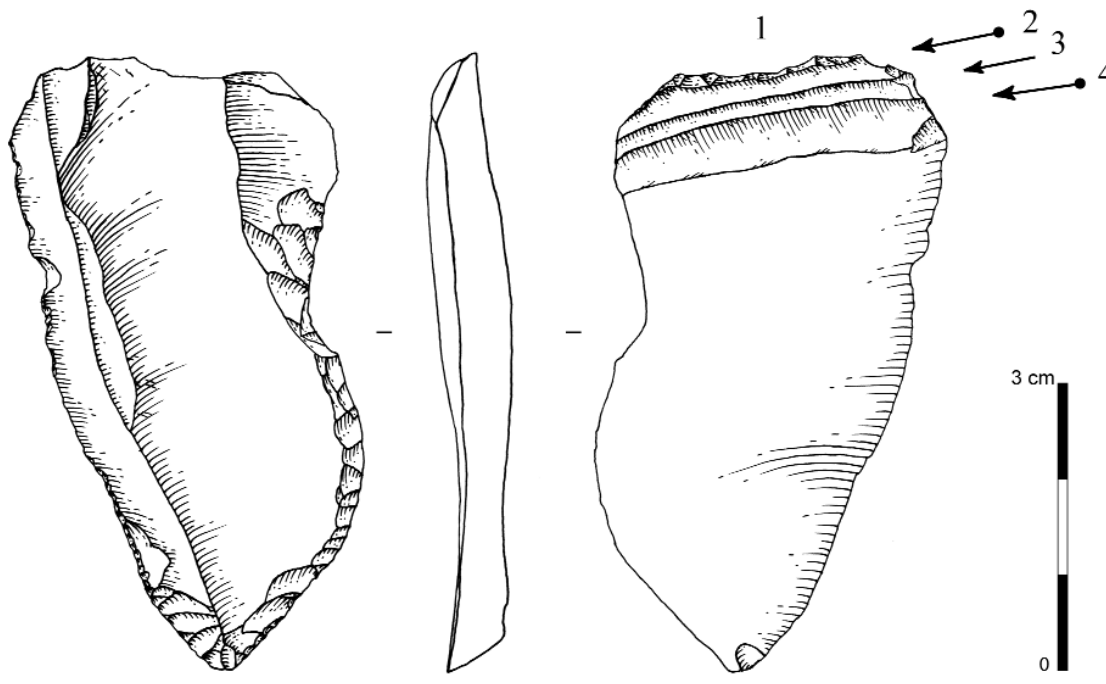

Figure 9 - Pièce de la Bertonne recueillie sur les bords de la Vézère, devant l'abri de la Rochette (commune de Saint-Léon-sur-Vézère,

département de la Dordogne, dessins L. Chehmana).

Figure 9 - La Bertonne artifact recovered on the banks of the Vézère in front of the Abri de La Rochette (commune of Saint-Léon-sur-Vézère, Department of the Dordogne, drawings by L. Chehmana). qui est recoupée systématiquement par un enlèvement lamellaire), nous avons cherché à savoir si elles ne résultaient pas, elles aussi, d'une production intentionnelle de lamelles. On verra que c'est cette hypothèse qui l'emporte au terme d'une argumentation que nous allons maintenant développer.

\section{Première hypothèse : les modifications de type "Bertonne " ont-elles été effectuées en vue d'un emmanchement ?}

C'est principalement la présence d'une extrémité active (fig. 11), essentiellement de type burin ou grattoir, sur la partie opposée à celle portant les modifications de type "Bertonne ", qui nous a conduit à nous interroger sur la fonction de ces aménagements. L'objectif des
Badegouliens était-il d'amincir ces extrémités afin de les insérer dans un manche (fig. 11a) ?

Dans cette optique, il nous semblait cependant curieux que les tailleurs aient envisagé un aménagement dissymétrique par rapport à l'axe longitudinal de la pièce (fig. 11b), plus exactement par rapport à l'axe déterminé par le positionnement de la partie supposée active. Cette première observation ne permettait pas, à elle seule, de rejeter entièrement l'hypothèse. Elle nous incitait simplement à rechercher l'existence d'éventuels autres faits incompatibles avec cette fonction.

Si nous avons finalement douté du bien-fondé de cette hypothèse, c'est parce que dans la collection du Trocadéro, ces extrémités actives ne sont présentes que 

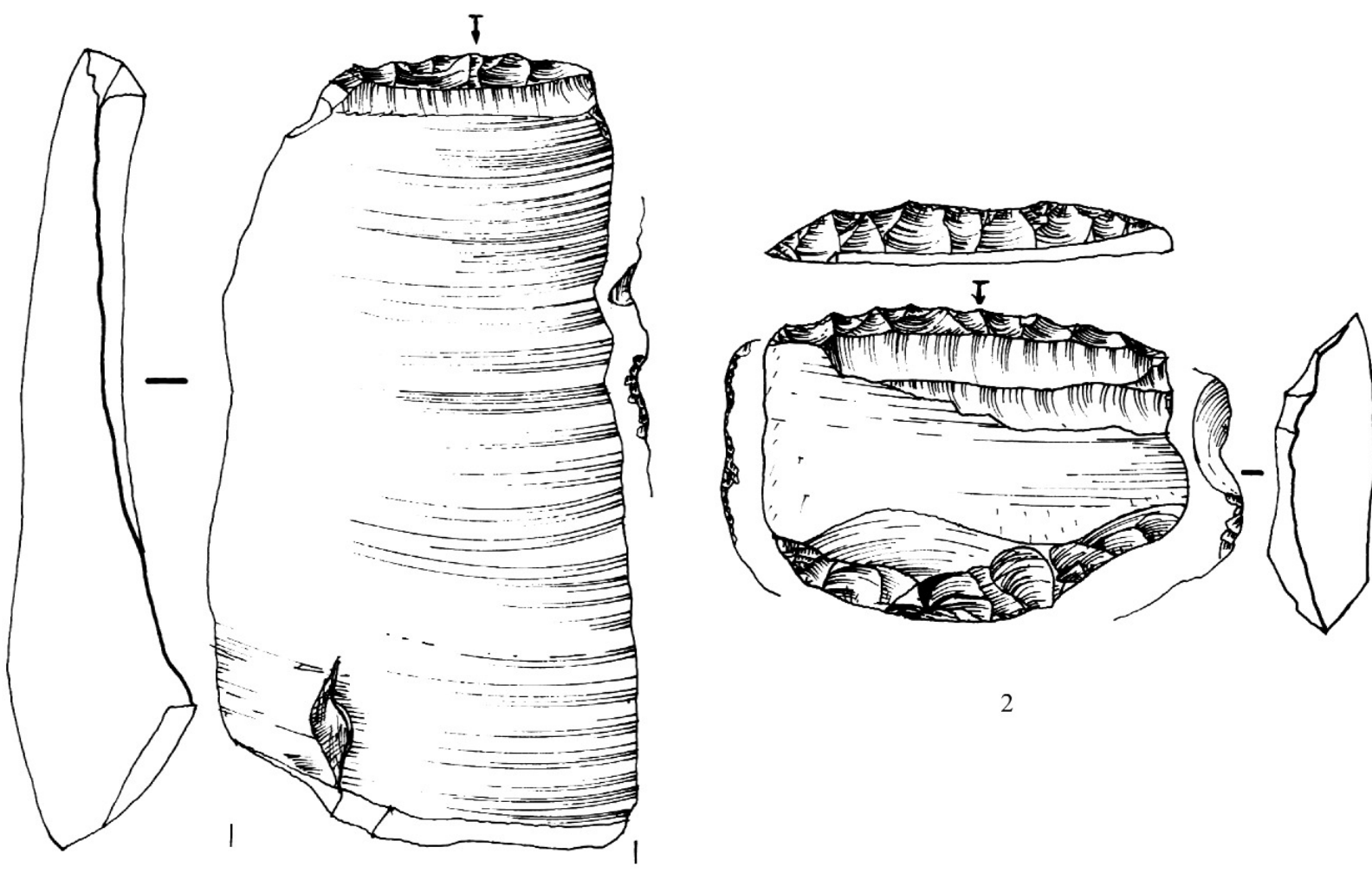

2

1
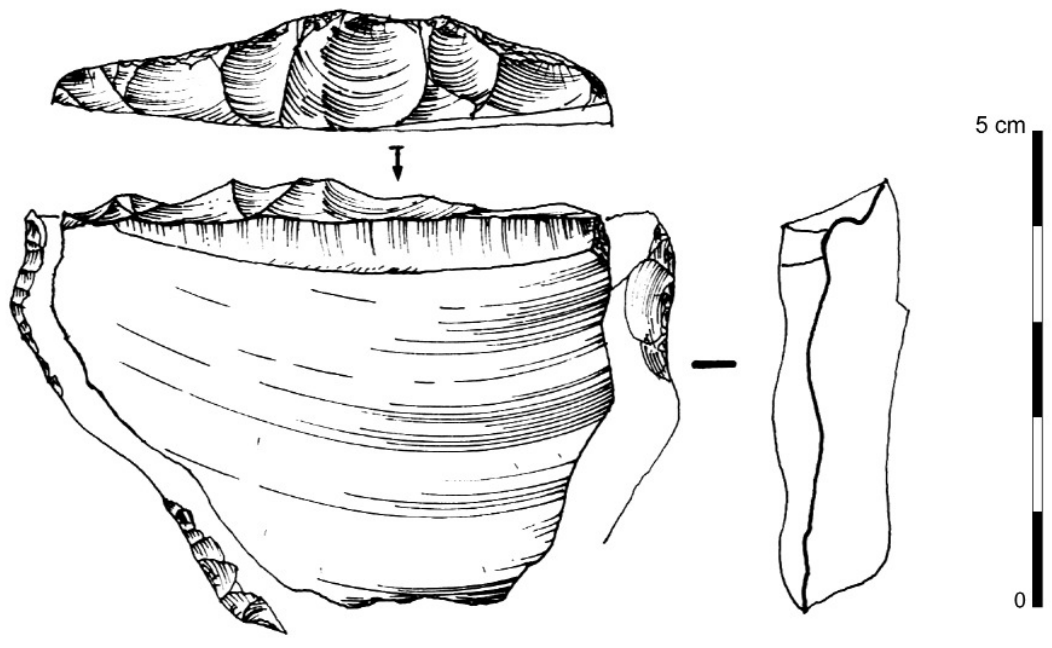

3

Figure 10 - Trois exemplaires de pièces d'Orville (collection Perlès, d'après dessins A. Moundrea dans Perlès, 1982 fig. 1, fig. 2 et fig. 3).

Figure 10 - Three examples of Orville artifacts (Perlès collection, after drawings by A. Moundrea in Perlès 1982, fig. 1, fig. 2 and 3). 
Extrémité présumée active

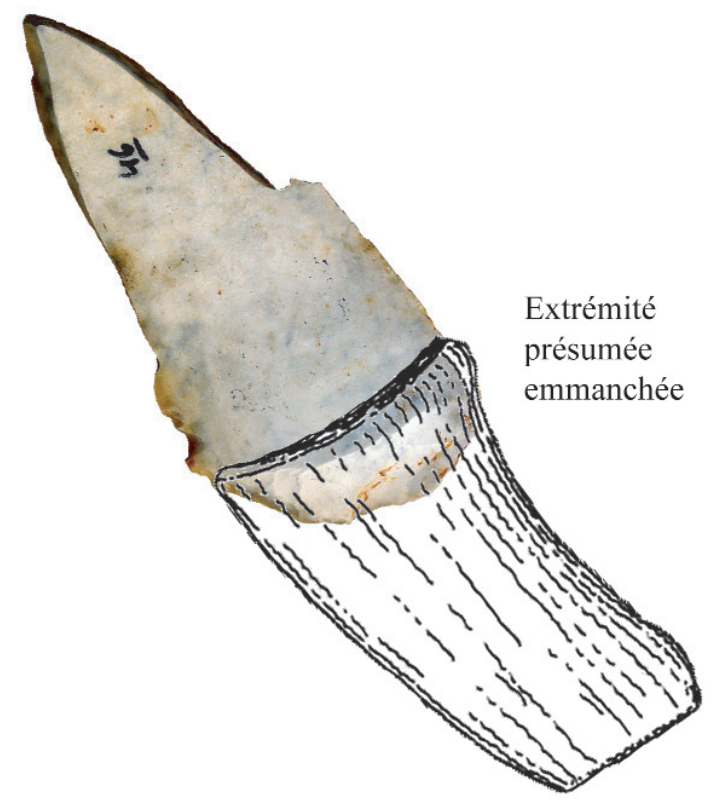

a. proposition d'emmanchement
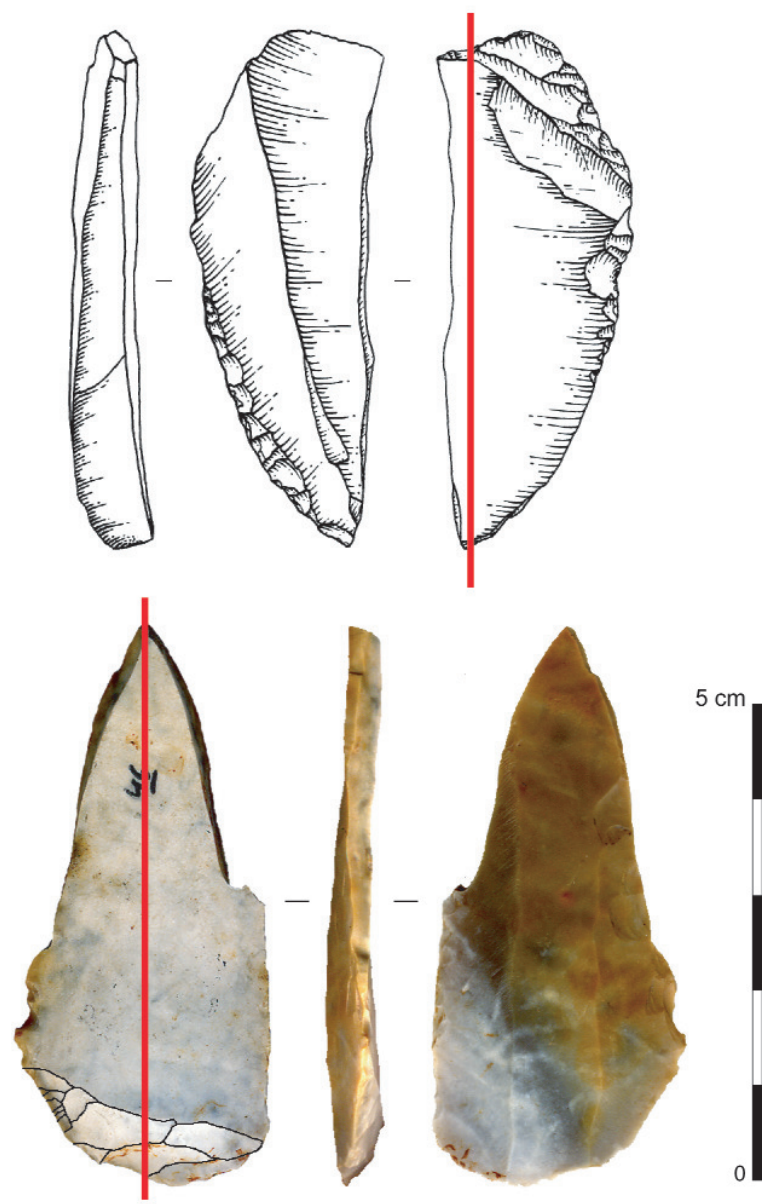

b. en rouge, l'axe longitudinal de la pièce

Figure 11 - Deux exemples de burin installé à l'extrémité opposée d'un aménagement de type "Bertonne » (collection Bazin, site du Trocadéro, DAO L. Chehmana).

Figure 11 - Two examples of burins on the end opposite to « La Bertonne »type preparation (Bazin collection, Trocadéro site, DAO, L. Chehmana).

\begin{tabular}{|c|c|c|c|c|c|}
\cline { 2 - 6 } \multicolumn{1}{c|}{} & Extrémité active & 2e aménagement de type Bert. & Extrémité cassée & Extrémité non aménagée & Total \\
\hline Effectif & 16 & 14 & $14(\mathrm{dt} 2$ volontairement $)$ & $23(\mathrm{dt} 7 \mathrm{avec}$ éperon) & 67 \\
\hline Pourcentage & 24 & 21 & 21 & 34 & 100 \\
\hline
\end{tabular}

Tableau 3 - Nature des extrémités opposées aux aménagements de type «Bertonne » (collection Bazin, site du Trocadéro).

Table 3 - Kinds of extremities opposite to La Bertonne preparation (Bazin collection, Trocadéro site).

sur $20 \%$ des pièces qui comportent un aménagement de type « Bertonne » (tabl. 3). De ce fait, leur présence n'est pas systématique.

La série contient par ailleurs de nombreuses pièces de la Bertonne doubles ( $\mathrm{N}=14$; fig. 12a). Cela signifie que le même type d'aménagement a été effectué aux deux extrémités des lames. Nous nous sommes alors demandés si les Badegouliens avaient pu concevoir les pièces emmanchées des deux côtés (fig. 12b). Dans ce cas, les bords tranchants seraient les seules parties actives restées appa- rentes. Notre tentative de modélisation par le dessin (fig. 12b) montre toutefois que ce procédé de double emmanchement n'est pas du tout ergonomique. D'autre part, on se demande pourquoi les Badegouliens auraient utilisé des tranchants si courts alors qu'ils recherchaient dans bien des cas de longues lames. Sur le site du Trocadéro, localisé à proximité de plusieurs affleurements de silex, les lames mesurent en moyenne entre 10 à $15 \mathrm{~cm}$ de long alors que la longueur des tranchants conservés sur les pièces de la Bertonne doubles ne dépasse jamais $4,5 \mathrm{~cm}$. 
En outre, certaines pièces de la Bertonne portent encore, à l'une de leurs extrémités, un talon en forme d'éperon proéminent et large (fig. 13). Lorsque l'on observe la modélisation, il est difficile d'imaginer que la partie opposée ait été aménagée en vue d'un emmanchement! Dans le cas contraire, les parties actives ne pouvaient être, là encore, que les bords tranchants des lames. Cette hypothèse s'est cependant révélée totalement inadaptée lorsque nous avons constaté que certains de ces aménagements avaient été effectués sur des éclats qui, emmanchés (fig. 14), auraient été insérés en quasi totalité. De plus, si cet amincissement avait vraiment été effectué en vue d'un emmanchement, pourquoi aménager, dans de nombreux cas (45\% sur le site du Trocadéro), ces futures zones d'emmanchement en partie distale du support alors qu'au Paléolithique supérieur, elle est souvent réservée pour l'extrémité active (Leroi-Gourhan et Brézillon 1972 p. 30 et 36 ; Bricker et David in Bricker 1995 - p. 94 ; Bricker in Bricker 1995 - p. 147 ; Brooks in Bricker 1995 - p. 205).

En somme, d'après ces différents arguments, il semble improbable que ces extrémités de lames aient été retouchées pour faciliter leur insertion dans un manche. Ainsi, il nous reste deux autres hypothèses à vérifier : outil et nucléus à lamelles ?

\section{Deuxième hypothèse : les modifications de type "Bertonne" forment-elles une partie active ?}

Afin de pouvoir tester cette hypothèse, une analyse tracéologique a été réalisée par l'un d'entre nous (N.H.) sur une dizaine de pièces sélectionnées dans la collection du Trocadéro. Bien que ces pièces soient recouvertes d'un voile opaque, des traces pouvant résulter d'une utilisation ont été conservées sur certaines d'entre elles et les premiers résultats obtenus sont donc plutôt encourageants. Pour résumer, aucun poli d'usure n'a été détecté sur les faces inférieures des pièces de la Bertonne, plus précisément à l'emplacement des négatifs lamellaires (fig.15b). En revanche, leur présence a été relevée sur la face supérieure de certaines d'entre elles $(\mathrm{N}=2)$. Elle a été décelée sur une surface très localisée : le long de la ligne délimitée par le départ des enlèvements lamellaires (fig. 15a). L'assez bon état de conservation des polis a même permis de déterminer que l'un de ces bords a été en contact avec une matière dure animale.

À partir de ces premières informations, nous avons ainsi pu imaginer le positionnement éventuel de l'outil sur la surface à travailler, l'action, le geste d'utilisation et enfin le type d'outil le plus probable. D'après la localisation des traces d'utilisation, s'il s'agit bien d'outils, les pièces devraient plutôt être positionnées face supérieure sur la surface à

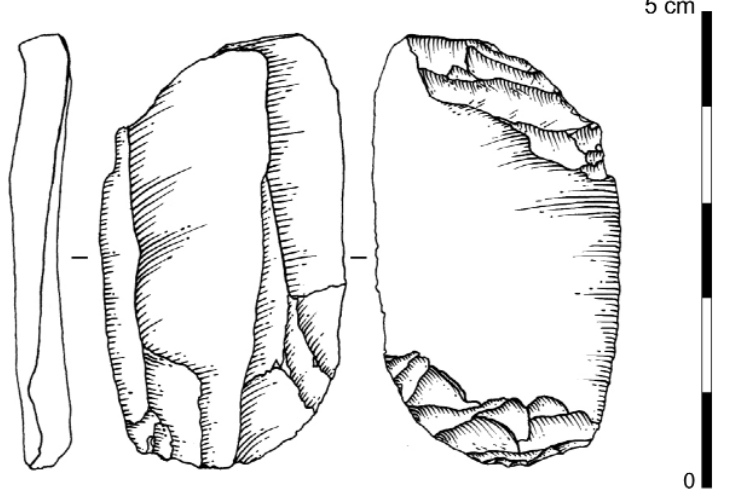

Figure 12 - Proposition d'emmanchement d'une pièce de la Bertonne double (collection Bazin, site du Trocadéro, DAO L. Chehmana).

Figure 12 - Hypothetical hafting of a double La Bertonne artifact (Bazin collection, Trocadéro site, DAO, L. Chehmana).

a. le Trocadéro : pièce de la Bertonne double

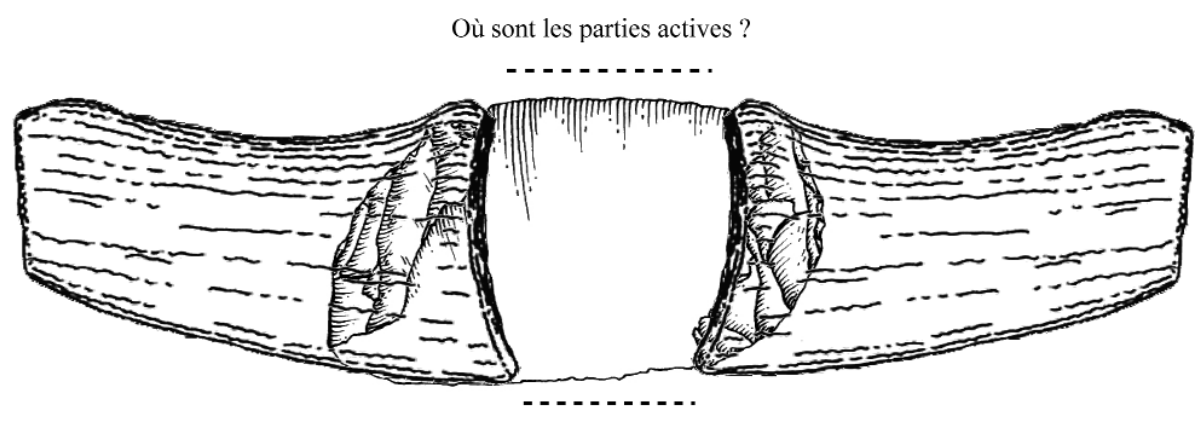

b. proposition d'emmanchement 


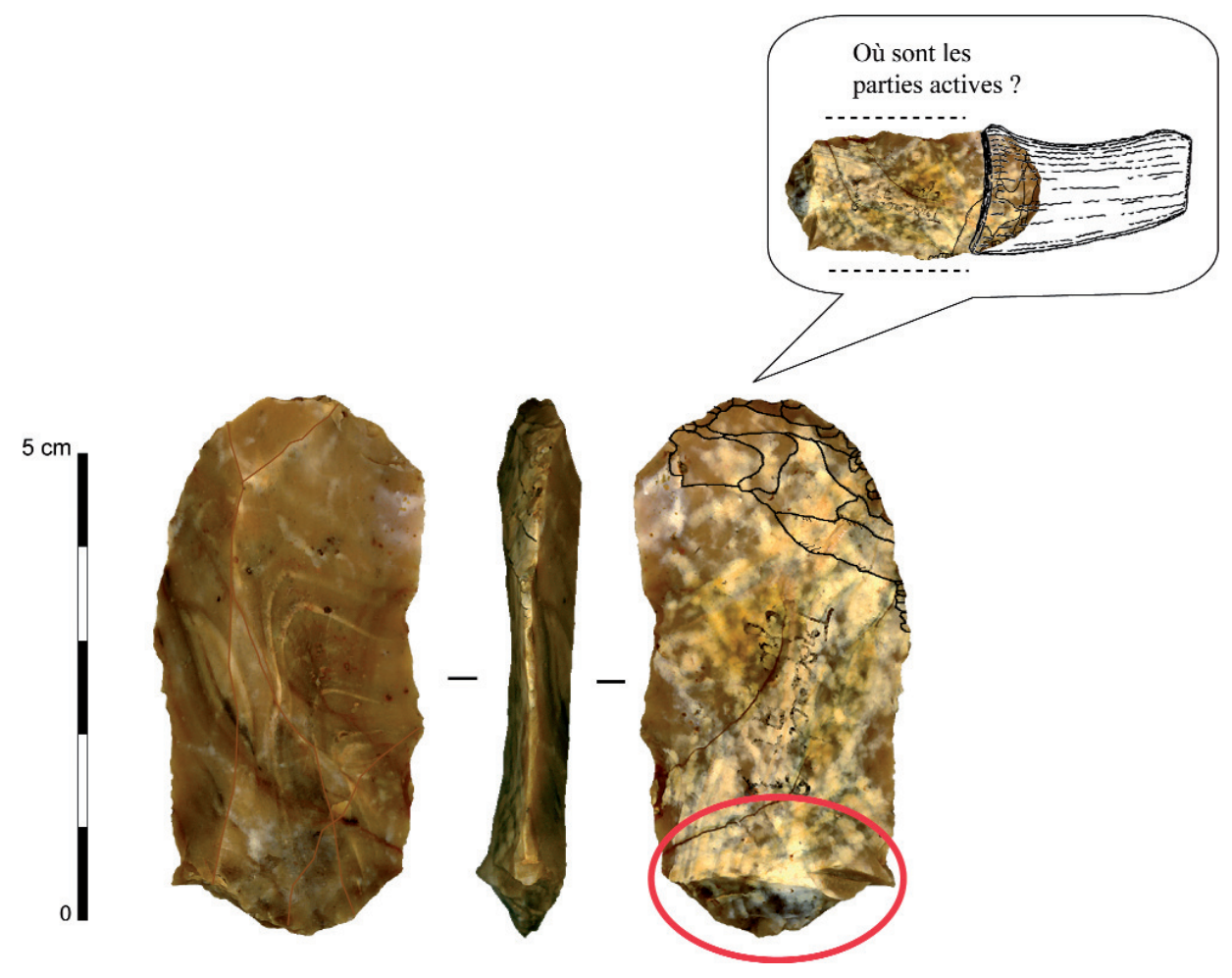

Figure 13 - Présence du talon sur une pièce de la Bertonne (collection Bazin, site du Trocadéro, DAO L. Chehmana). Figure 13 - Presence of a platform on a La Bertonne artifact (Bazin collection, Trocadéro site, DAO, L. Chehmana).

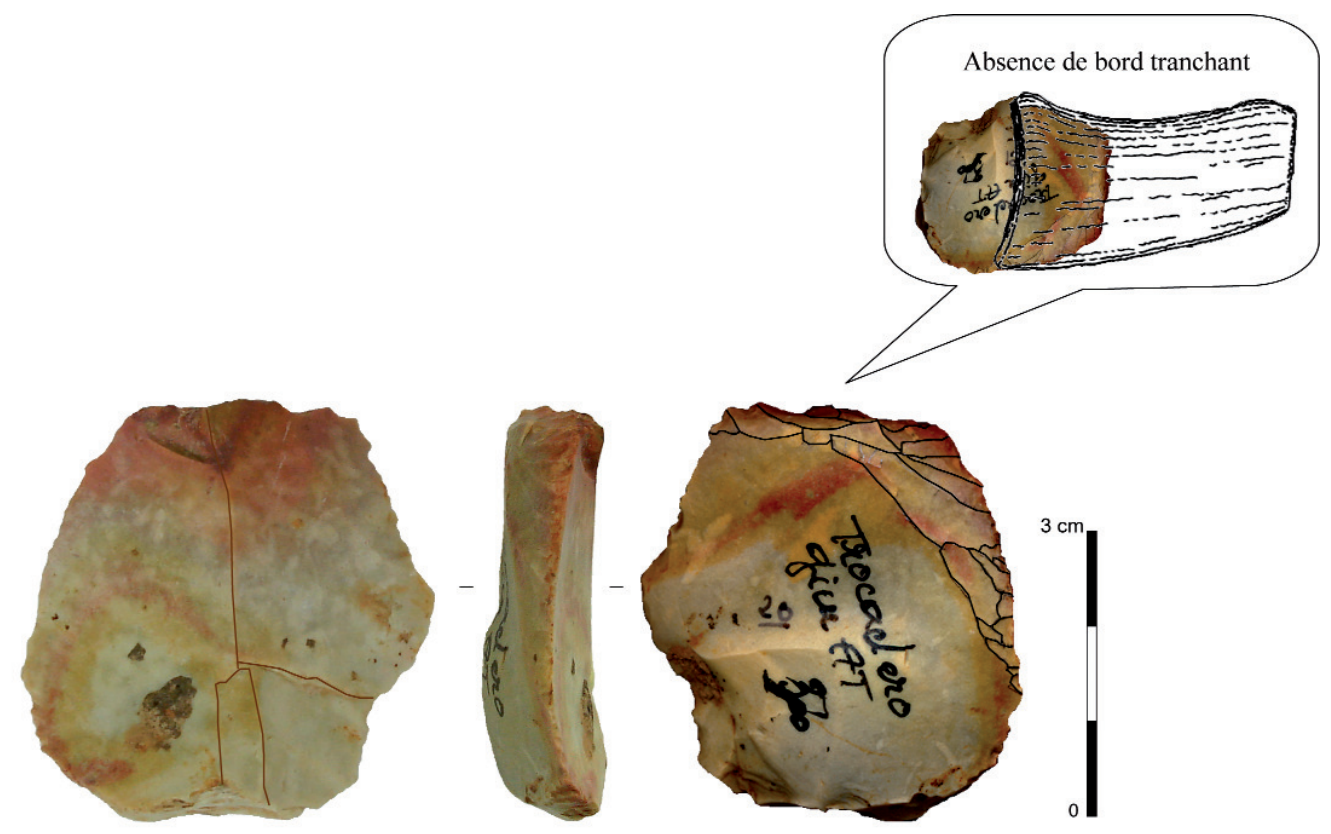

Figure 14 - Un aménagement de type "Bertonne » sur éclat (collection Bazin, site du Trocadéro, DAO L. Chehmana). Figure 14 - La Bertonne type preparation on a flake (Bazin collection, Trocadéro site, DAO, L. Chehmana). 
Des polis

sur la face supérieure
Aucun poli

sur la face inférieure

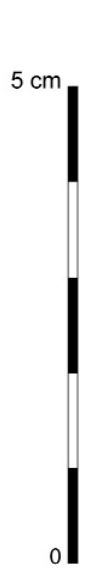

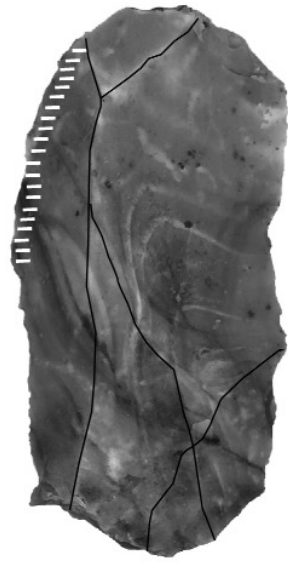

a.
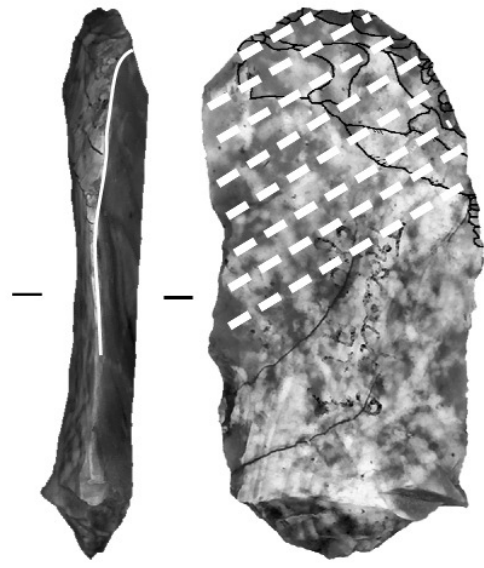

b.
Figure 15 - Résultat de l'analyse tracéologique (collection Bazin, site du Trocadéro, DAO L. Chehmana).

Figure 15 - Result of use-wear analysis (Bazin collection, Trocadéro site, DAO, L. Chehmana).

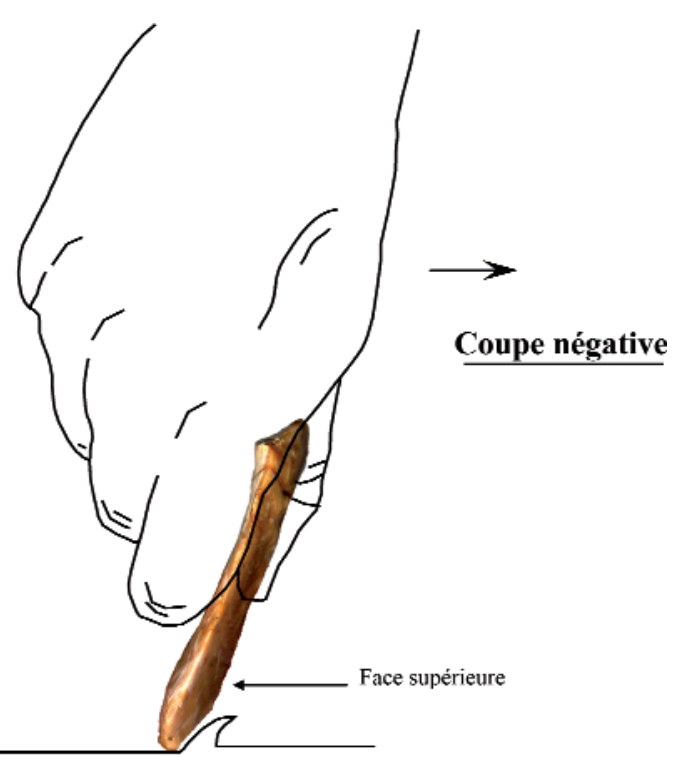

Figure 16 - Hypothèse d'utilisation d'une pièce de la Bertonne (collection Bazin, site du Trocadéro, DAO L. Chehmana).

Figure 16 - Hypothesis for the use of a La Bertonne artifact (Bazin collection, Trocadéro site, DAO, L. Chehmana).

travailler (fig. 16) et l'action réalisée serait le raclage. Le travail devrait s'effectuer transversalement au fil - constituant la ligne de rencontre de la face supérieure et inférieure du support, à l'emplacement du départ des enlèvements lamellaires - et probablement en coupe négative (fig. 16), c'est-à-dire vers soi. La présence d'esquillements uniquement sur la face inférieure (fig. 17) ne serait pas incompatible avec ce type d'utilisation. L'hypothèse du " grattoir inverse » proposée par M. Lenoir ne serait donc pas à exclure.
Nous avons toutefois remarqué sur plusieurs exemplaires de pièces de la Bertonne (fig. 18 à 21) qu'aucun esquillement n'apparaissait sur la ligne de départ du dernier enlèvement lamellaire. Cela signifie que ces pièces n'ont pas été utilisées après la dernière modification dont elles ont fait l'objet. Ce constat nous a alors conduit à émettre l'hypothèse qu'au moins cette ultime action avait pour but de détacher une lamelle.

\section{Troisième hypothèse : les modifications résultent-elles d'une production intentionnelle de lamelles ?}

Pour vérifier cette hypothèse, il a fallu rechercher ce qui pouvait correspondre à l'objectif éventuel de la production, c'est-à-dire les lamelles retouchées qui auraient été incontestablement produites sur des pièces de la Bertonne. Compte tenu des conditions de récoltes (ramassages de surface), pratiquement aucune des collections à pièces de la Bertonne n'en comportait. Deux font pourtant exception, celles de la Malignière et de Bellevue ! Ces séries étant composées de plusieurs ensembles archéologiques, la fabrication des lamelles avait, dans un premier temps, été imputée à des tailleurs de la fin du Magdalénien (Demars 1985). Nous avons toutefois constaté, à partir des dessins de ces lamelles, que la face supérieure de certaines d'entre elles portait des négatifs d'enlèvements transversaux envahissants qui pouvaient résulter de l'aménagement d'une troncature inverse sur le nucléus (fig. 22). Ces lamelles pouvaient donc avoir été détachées à partir de pièces de la Bertonne. Pour le démontrer, il fallait encore vérifier que ces négatifs ne soient ni le résultat d'une retouche, ni les parties vestigiales de l'aménagement d'une crête à un pan (fig. 23).

\section{Découverte récente de quelques lamelles retouchées obtenues à partir de pièces de la Bertonne}

Dans les collections de la Malignière et de Bellevue, cinq fragments (fig. 24) ont été isolés au sein d'un échantillon de 


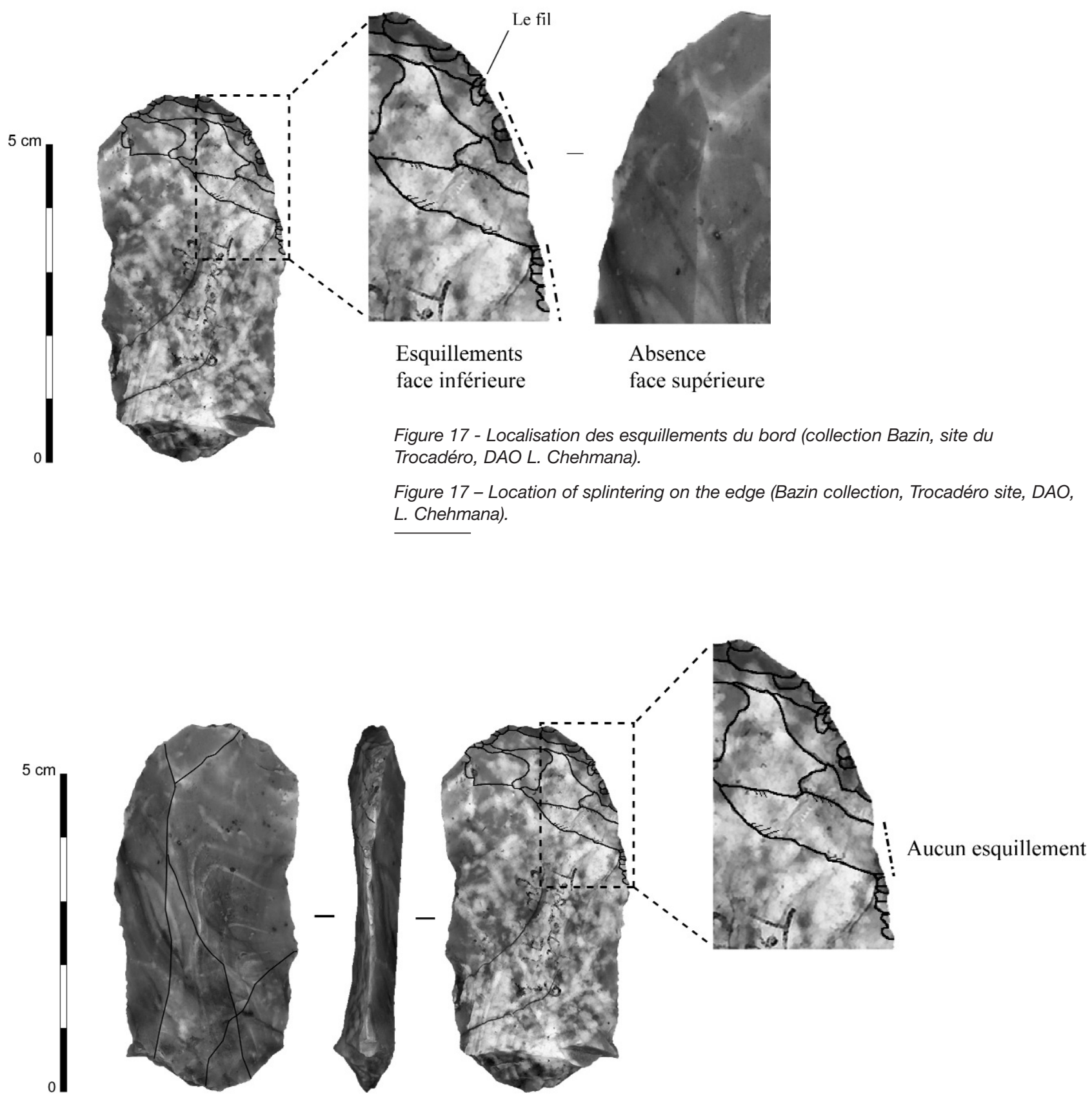

Figure 18 - Visualisation d'un dernier négatif d'enlèvement (collection Bazin, site du Trocadéro, DAO L. Chehmana).

Figure 18 - Visualization of a final removal scar (Bazin collection, Trocadéro site, DAO, L. Chehmana).

lamelles retouchées. Plusieurs détails techniques montrent qu'ils ont été incontestablement détachés à partir de pièces de la Bertonne :

- les négatifs d'enlèvements transversaux envahissants, visibles sur la face supérieure de ces fragments lamellaires, résultent d'un aménagement antérieur au détachement de la lamelle et non d'une modification liée à la retouche ; - par ailleurs, l'orientation de ces négatifs révèle qu'ils proviennent de l'aménagement d'une troncature et non d'une crête. Les ondes sont orientées du bord vers le centre de la lamelle (fig. 23a), à l'inverse donc, de celles visibles sur les négatifs qui résultent de l'installation d'une crête (fig. 23b) ;

- la reconnaissance d'une surface « positive » (ou panrevers in Klaric et al. 2002) sur l'autre partie de la face supérieure de certaines lamelles (fig. $24 n^{\circ s} 1$ et 2) permet de déterminer qu'elles ont aussi été détachées sur le revers d'un support. 

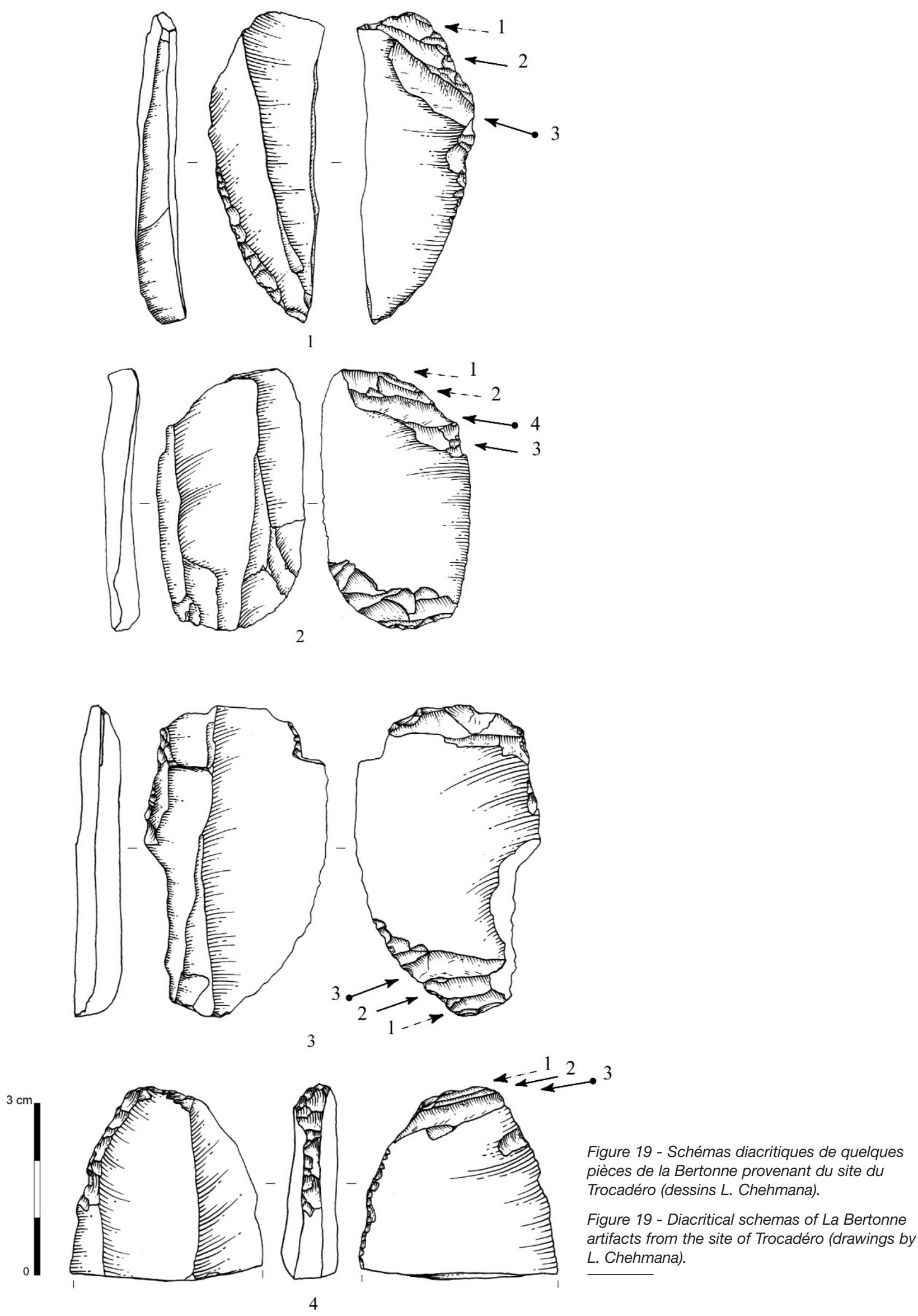

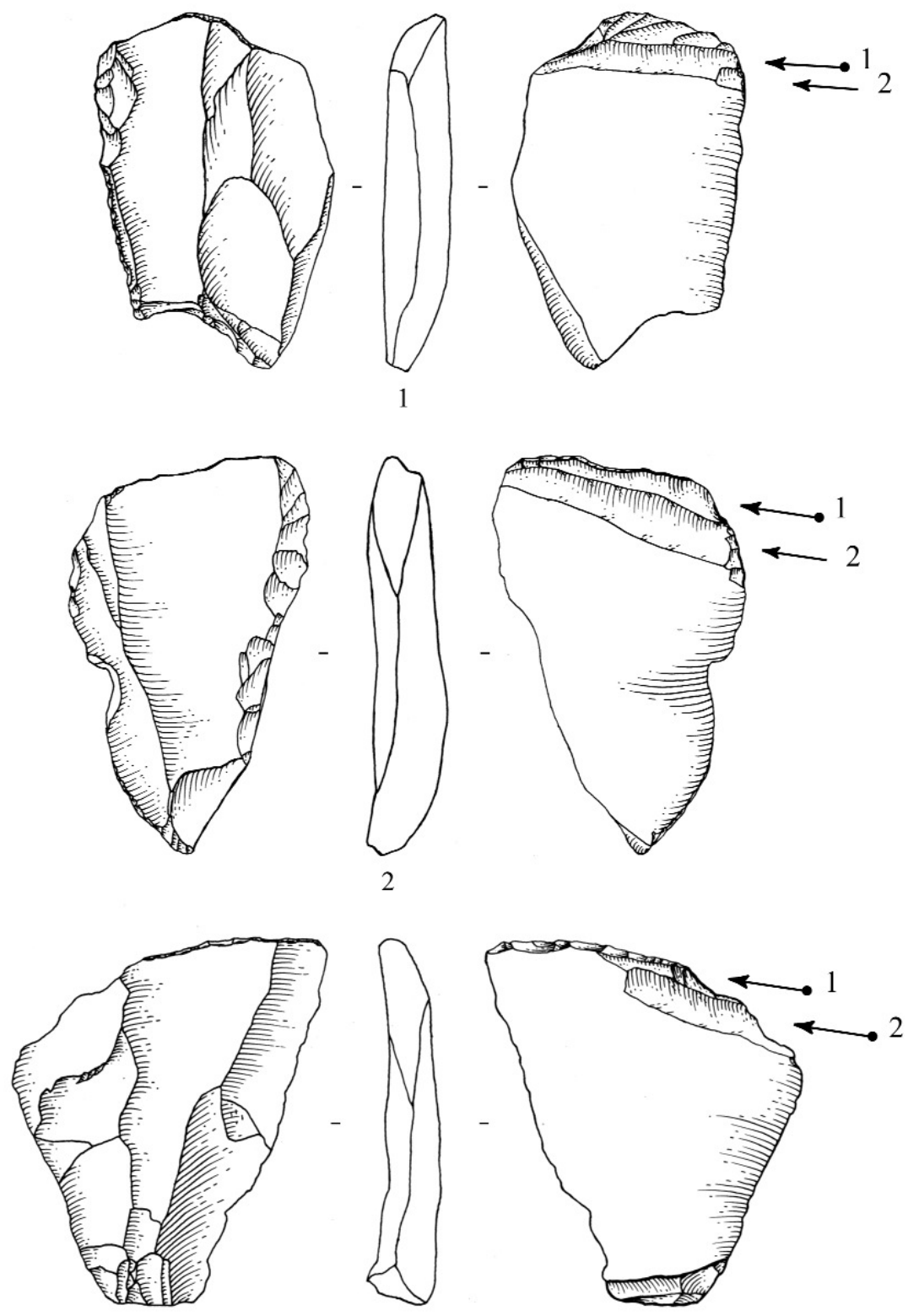

3
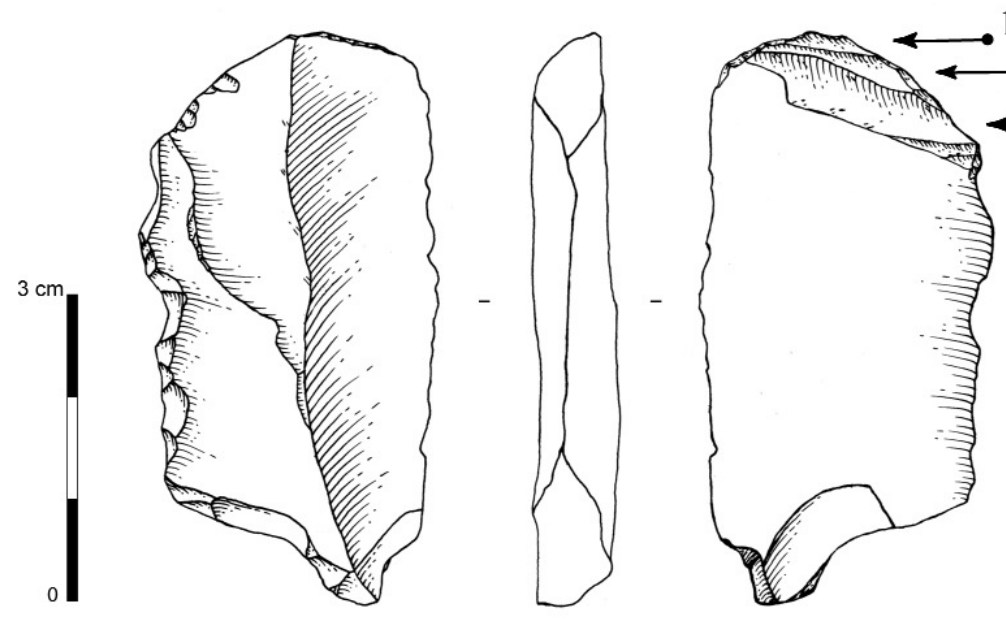

2

Figure 20 - Schémas diacritiques de quelques pièces de la Bertonne provenant du site de la Bertonne (dessins L. Chehmana).

Figure 20 - Diacritical schemas of La Bertonne artifacts from the site of La Bertonne (drawings by L. Chehmana). 

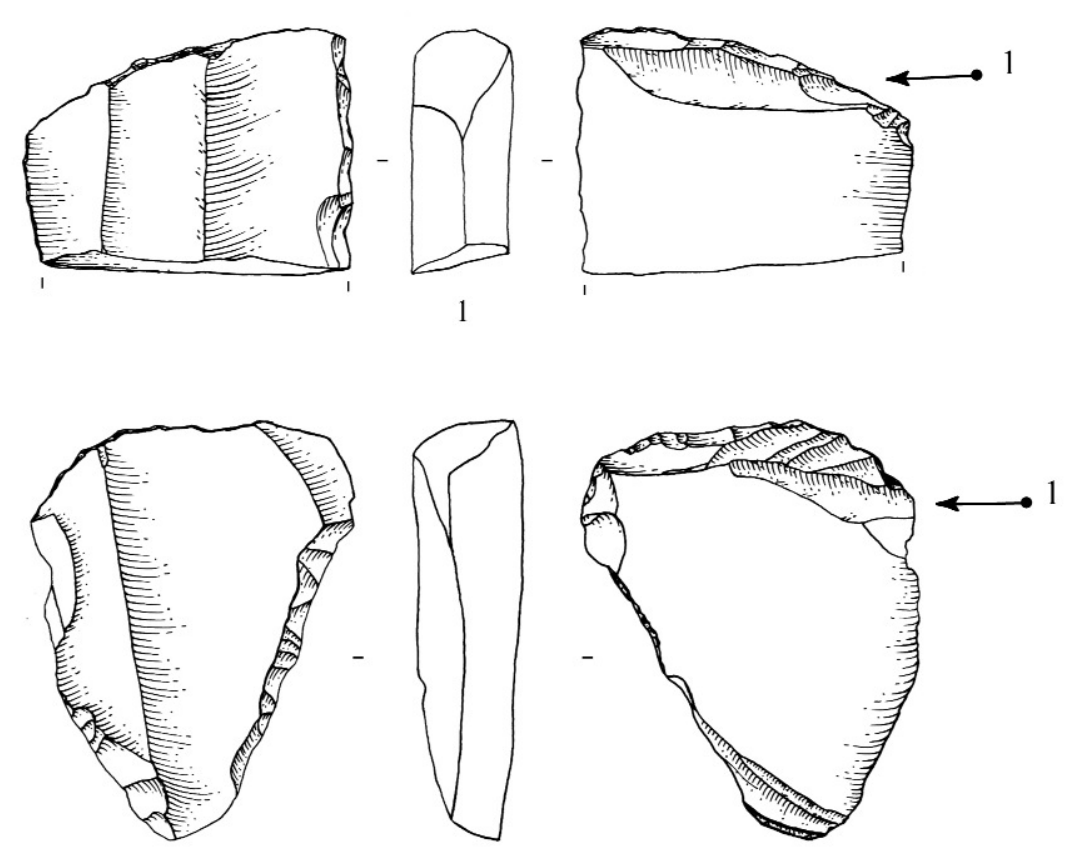

2
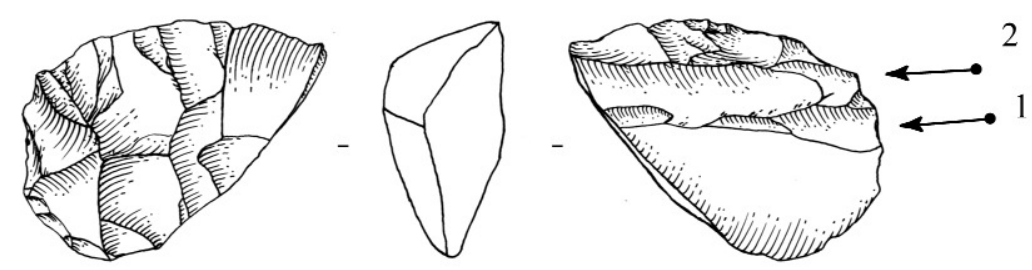

3
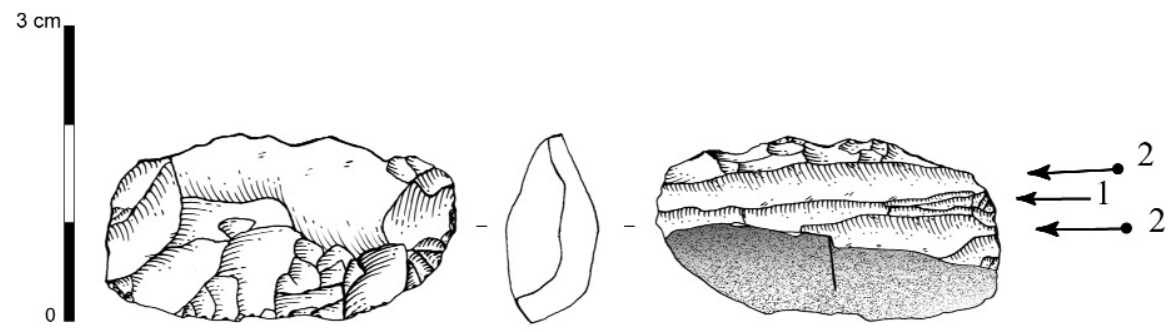

4

Figure 21 - Schémas diacritiques de quelques pièces de la Bertonne provenant du site de la Malignière (dessins L. Chehmana).

Figure 21 - Diacritical schemas of La Bertonne artifacts from the site of La Malignière (drawings by L. Chehmana).

Ainsi, ces trois détails techniques nous permettent d'affirmer, et ce malgré le faible nombre de produits retrouvés, que les Badegouliens ont volontairement détaché des lamelles à partir de pièces de la Bertonne (fig. 25).

Les cinq fragments lamellaires (fig. 24) ont été retouchés systématiquement à partir du bord droit : celui qui présente les extrémités distales de la troncature inverse. La retouche, principalement directe, réduit légèrement la largeur du support (4 cas sur 5) et semble surtout destinée à rectifier la délinéation du bord. Celui qui est opposé ne pré- sente aucun aménagement et conserve son fil tranchant. L'armature confectionnée correspond donc à une simple lamelle à dos.

En mesurant les négatifs d'enlèvements lamellaires sur les nucléus, nous avons pu en déduire les dimensions probables des lamelles recherchées - entre 13 et $30 \mathrm{~mm}$ de long et 3 et $7 \mathrm{~mm}$ de large - (fig. 26). Les tailleurs obtenaient dans la plupart des cas des gabarits de lamelles variables au sein d'une même séquence opératoire. Des lamelles longues et rectilignes ont d'abord été détachées puis, progressive- 
(Collection Lasnier, dessins Lasnier)
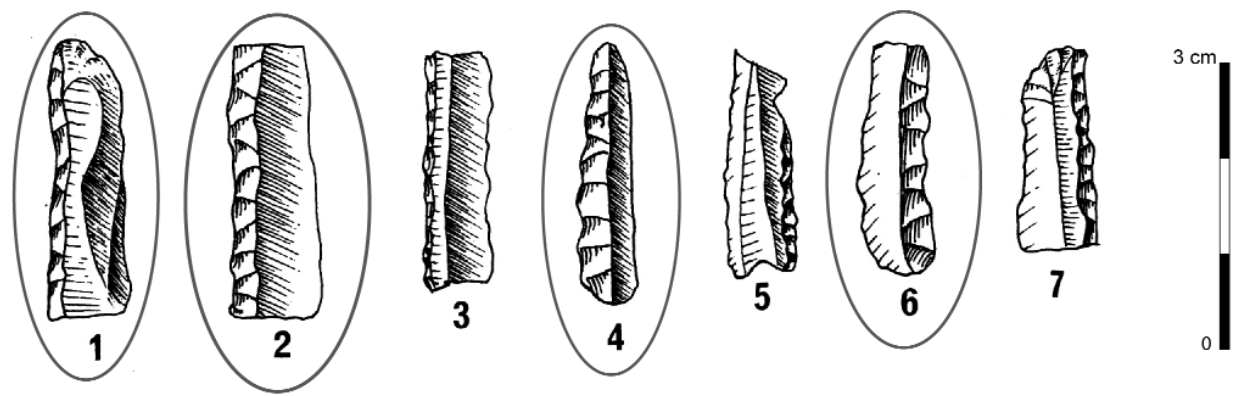

(Collection Lasnier, dessins Demars)
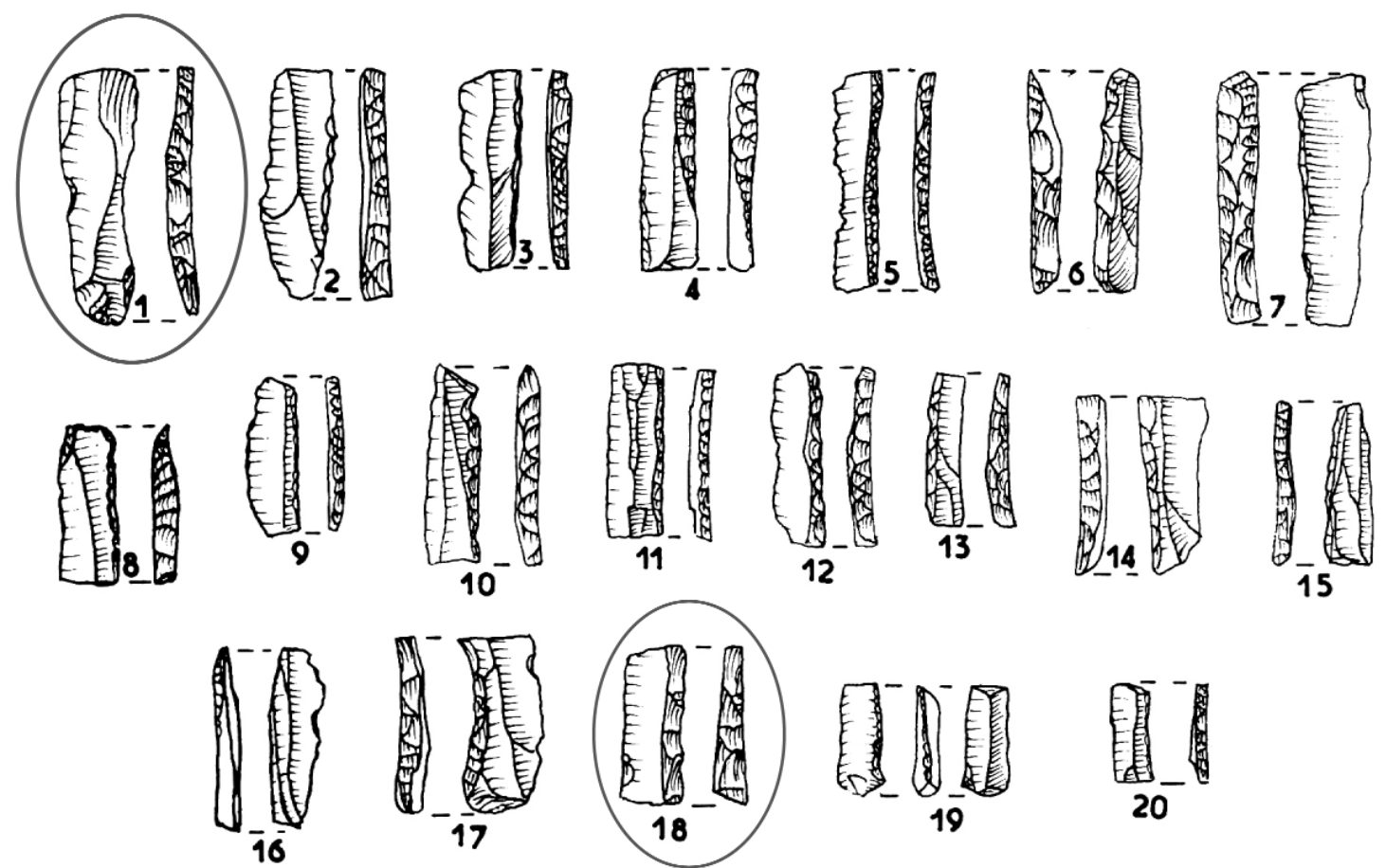

Figure 22 - Lamelles retouchées recueillies sur le site de la Malignière (d'après Delporte 1968 p. 440 ; Demars 1985 (fig. 5). Celles qui sont encerclées présentent des négatifs d'enlèvements transversaux envahissants.

Figure 22 - Retouched bladelets from the site of La Malignière (after Delporte 1968, p. 440; Demars 1985, fig. 5). The circled pieces have invasive transversal removal scars.

ment, des lamelles courtes, courbes et extrêmement minces. À l'heure actuelle, ces dernières n'ont pas encore été diagnostiquées dans les séries à pièces de la Bertonne.

Selon l'un d'entre nous (J.-P.), les lamelles obtenues à partir des pièces de la Bertonne ont par ailleurs été détachées par pression. Ainsi, la reconnaissance de cette technique de détachement sera à l'avenir essentielle pour identifier l'emploi de la méthode de débitage de lamelles de type « Bertonne ».

\section{La question de la technique de détachement des lamelles (J.P.)}

On a vu que cette interrogation a été très tôt évoquée par Leyssales et Noone (1949), sans doute en connaissance des essais de retouche par pression de L. Coutier, lors de leur description des pièces de la Bertonne du Pech de Saint-Sourd : «... série de longues cannelures transversales et plus ou moins parallèles résultant de l'enlèvement de lamelles par une pression ou par percussion quel- 
Lamelle "Bertonne"

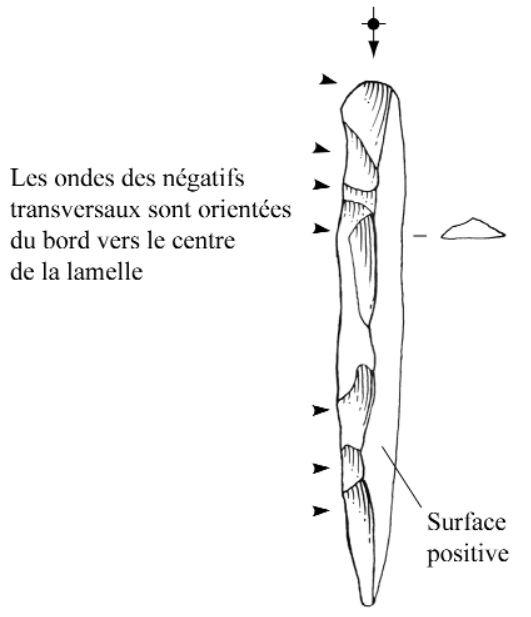

a.

Lamelle sous-crête

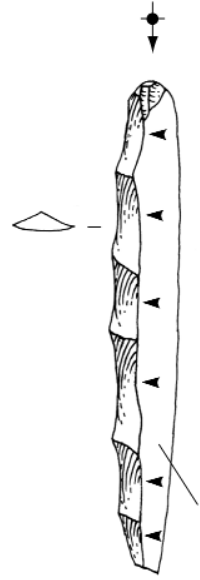

b.
Les ondes des négatifs

transversaux sont orientées

du centre vers le bord

de la lamelle
Figure 23 - Critères de distinction entre une lamelle "Bertonne » et une lamelle sous-crête (DAO

L. Chehmana).

Figure 23 - Criteria for differentiating between a « La Bertonne » bladelet and a bladelet under crest (DAO, L. Chehmana).

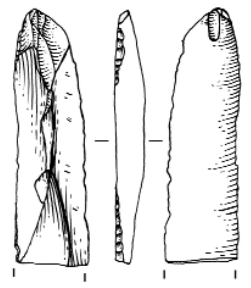

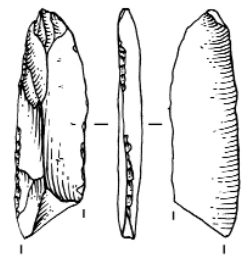

2

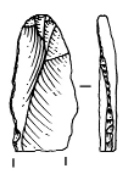

3

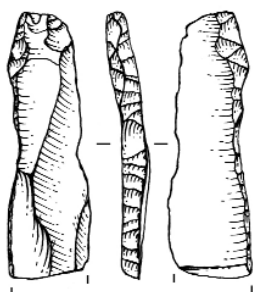

4

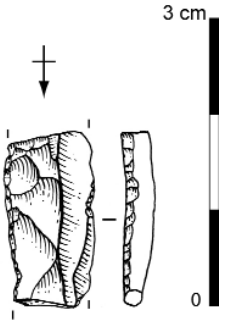

Figure 24 - Lamelles retouchées dont les supports ont été obtenus incontestablement à partir de pièces de la Bertonne (collection Lasnier). $n^{\circ} 1$ : site de Bellevue; $n^{\circ s} 2$ à 5 : site de la Malignière (dessins L. Chehmana).

Figure 24 - Retouched bladelets for which the blanks were unquestionably obtained from La Bertonne artifacts (Lasnier collection). $n^{\circ}$ 1: Bellevue site; $n^{\circ} 2-5$ : site of La Malignière (drawings by L. Chehmana).

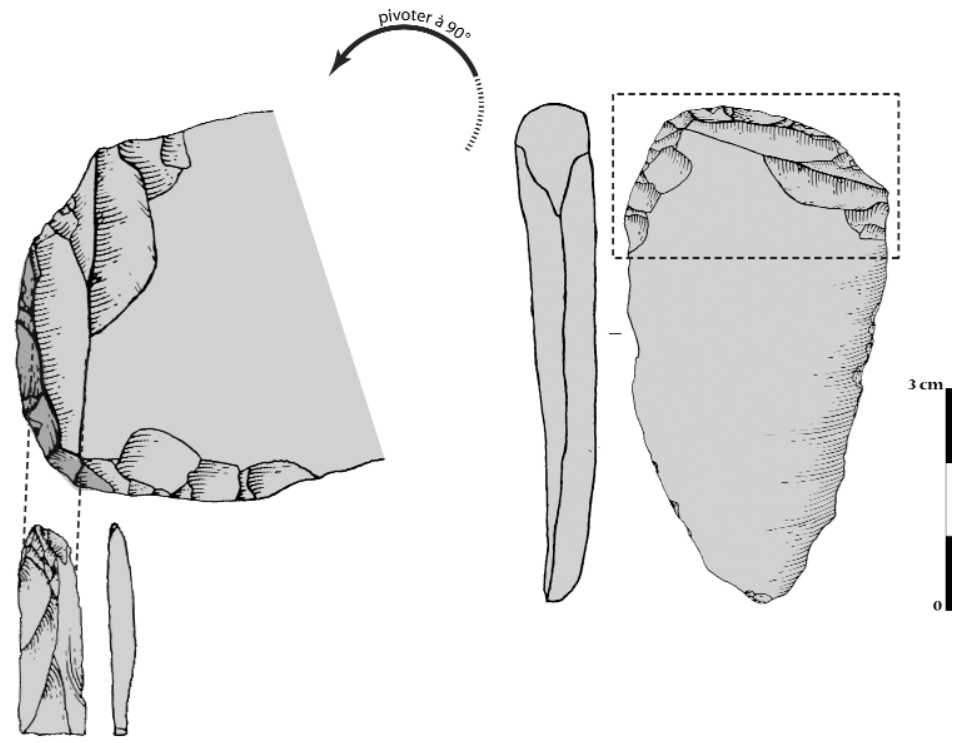

Figure 25 - Schéma d'obtention de lamelle à partir d'une pièce de la Bertonne (DAO L. Chehmana).

Figure 25 - Schema for bladelet production from a La Bertonne artifact (DAO, L. Chehmana). 


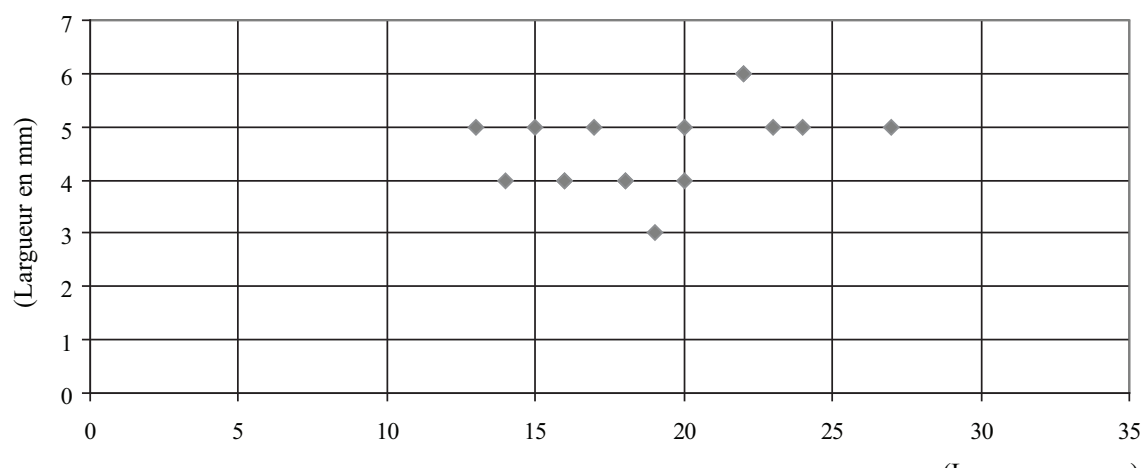

La Bertonne

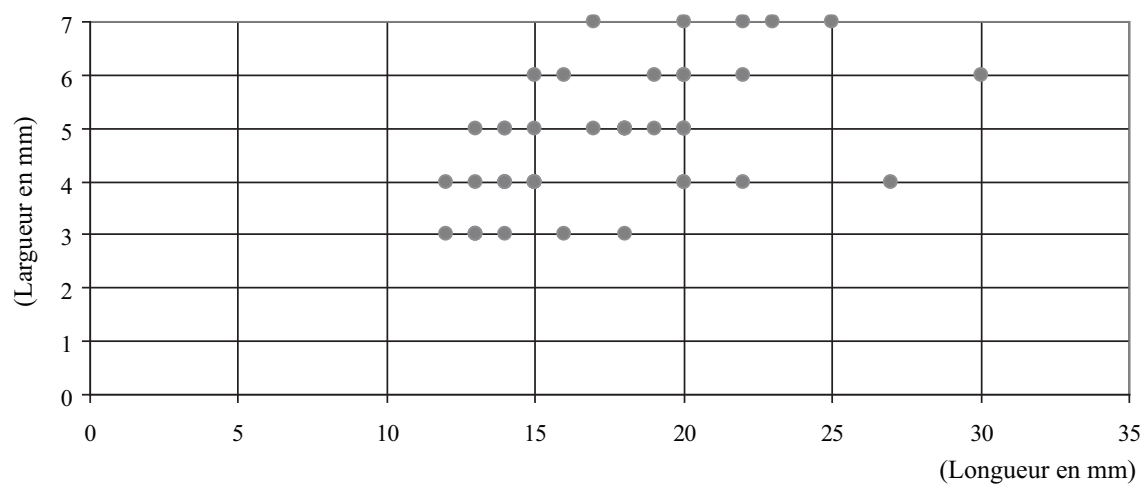

Le Trocadéro

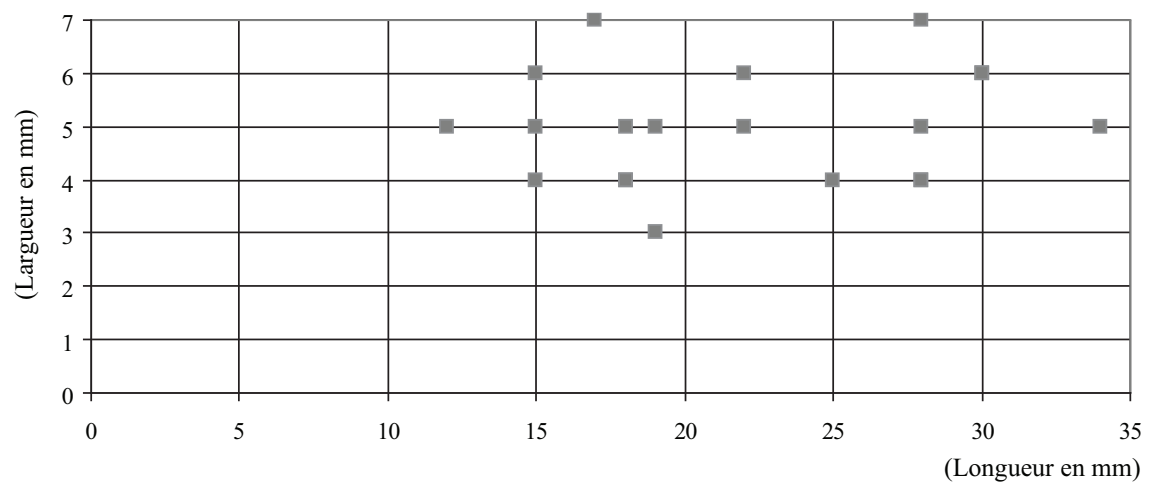

La Malignière

Figure 26 - Comparaison des longueurs et largeurs des négatifs lamellaires entiers mesurées sur les pièces de la Bertonne de trois séries.

Figure 26 - Comparison of lengths and widths of whole bladelet scars measured on La Bertonne artifacts from three assemblages.

conque exercée latéralement sur la face supérieure gauche de la lame » (p. 251).

Les pièces d'Orville posaient la même alternative (Perlès 1982 - p. 132), et nos premiers essais de l'époque s'étaient contentés de montrer que la percussion directe tendre - avec un petit percuteur en bois de renne de $200 \mathrm{~g}$ pouvait reproduire l'essentiel des détachements visibles sur les pièces archéologiques (Pelegrin 1982). En défaveur de la pression - à laquelle nous n'étions pas encore très entraîné, certains négatifs d'enlèvements archéologiques atteignaient $10 \mathrm{~mm}$ de large, en limite du possible par pression ( $8 \mathrm{~mm}$ dans nos essais), et certaines pièces d'Orville portaient la trace d'un esquillement ou même d'un enlèvement parasite au niveau du point d'origine de l'enlèvement lamellaire, témoignant en faveur d'un accrochage de la corniche par un outil lancé.

En 1995, nous avons procédé à un test plus documenté en soutien du mémoire de Maîtrise d'O. Parisot (1995 - p. 35 
et 36 fig. 37b), en produisant 15 enlèvements lamellaires par percussion directe tendre et 19 autres par pression, ces derniers les plus larges possibles (à la béquille d'épaule). Les « nuages de points » respectifs se recouvraient largement, le nuage " percussion » débordant du nuage " pression » quant à la largeur (au-delà de $8 \mathrm{~mm}$ ) et aussi quant à la longueur (au-delà de $4,5 \mathrm{~cm}$ ). Les négatifs de lamelles archéologiques "Orville » atteignant $10 \mathrm{~mm}$ de largeur (avec 1 cas à 14) et jusqu'à plus de $6 \mathrm{~cm}$ de longueur, il en a été déduit que la percussion avait certainement été utilisée, mais que l'on ne pouvait exclure l'usage de la pression.

Les enlèvements lamellaires « Bertonne » étant assez nettement plus petits que les «Orville ", nous avons cette fois complété ces tests en essayant encore de réaliser par percussion les enlèvements les plus étroits possible. Ce complément porte le corpus actuel à 32 détachements par percussion directe tendre, avec une dizaine d'essais supplémentaires par pression.

Par pression, la largeur obtenue reste de 3 à $8 \mathrm{~mm}$, pour une longueur de 2,5 à 4,5 cm (surtout 4 à $7 \mathrm{~mm}$ de large et 2,5 à $4 \mathrm{~cm}$ de long).

Par percussion, la largeur s'étend de 4 à $11 \mathrm{~mm}$, pour une longueur de 1 (réfléchi) à $6,5 \mathrm{~cm}$ (surtout 4 à $9 \mathrm{~mm}$ de large et 2 à $6 \mathrm{~cm}$ de long).

Cette fois, au vu de ces seuls critères morphologiques (tabl. 4), les deux techniques apparaissent capables de recouvrir la dispersion archéologique des pièces de la Bertonne (fig. 26). Comme pour les techniques de débitage de lames et de lamelles, les seuls arguments morphométriques sont donc insuffisants ou inadéquats pour établir un diagnostic. Mais un examen plus qualitatif des stigmates permet de pencher nettement en faveur de l'usage de la pression pour les pièces de la Bertonne.

Effectués par séries de 2, 3 voire 4 enlèvements successifs, en règle depuis l'arête formée par la troncature en étendant la surface débitée vers la face inférieure du support, ces enlèvements "Bertonne » sont nettement minces, tout en restant étroits et parfois très réguliers, atteignant le bord opposé sur les pièces les plus « réussies » (fig. 27 nos 5, 8 et 9). Un tel contrôle répété, possible par pression (fig. $28 n^{\circ} 1$ ), paraît vraiment impossible par percussion : la colonne de silex que représente la lamelle détachée est si mince que, par percussion, elle se brise quasiment systématiquement pendant son détachement, se terminant alors par des rides et un réfléchissement (ce serait un défi de détacher plus de deux tels enlèvements minces et réguliers par percussion) ;

$\mathrm{Si}$, justement, beaucoup des enlèvements expérimentaux par percussion se terminent par un réfléchissement ou une amorce de réfléchissement, l'aspect de ce réfléchissement est différent de celui des pièces archéologiques "Bertonne », ce dernier étant plus proche de ce que nous avons obtenu par pression. Le réfléchissement le plus fréquemment observé sur les pièces expérimentales par percussion est en « rebroussé » (fig. $28 n^{\circ} 7$ ) ou amorce de rebroussé (fig. $28 n^{\circ} 9$ ). Le premier (7) est clairement déterminé par l'effet de recul de la pièce sous l'impact du coup, le second (9) est probablement dû à la fois à la fracture proximale de la lamelle détachée (marquée par l'apparition soudaine de rides à mi-parcours, c'est à dire durant le détachement : cas aussi de la fig. $28 \mathrm{n}^{\circ} 2$ ), et à l'impossibilité de prolonger son élargissement en partie distale (on sait d'ailleurs que, par pression, des enlèvements lamellaires tendent très rarement à s'élargir dans leur moitié distale : au mieux, ils conservent des bords parallèles). Les réfléchissements observés sur les pièces archéologiques (fig. 27), à y regarder de près, sont d'un aspect le plus souvent différent. Ils sont en " marche " (ou step fracture des anglo-saxons qui distinguent cette dernière forme de la hinge termination en arrondi), selon un autre mécanisme : la colonne de silex se brise, mais l'effet de pression produit encore une terminaison en « marche » (comme dans certains accidents d'usage en armature), voire un petit enlèvement supplémentaire (fig. $27 \mathrm{n}^{\circ \mathrm{s}} 1$ et 3 , fig. $28 \mathrm{n}^{\circ \mathrm{s}} 5$ et 6 ), ou encore une amorce d'un tel enlèvement sous forme d'une fissure incipiente dans l'épaisseur du support. Cet aspect de fracture en marche semble être aggravé par une compression modérée exercée sur la surface de débitage pendant le détachement, en rapport avec la tenue en main de la pièce (les pièces fig. $28 n^{\circ s} 4$ à 6 , les plus nettes de ce point de vue, ont été tenues dans la paume protégée par de la peau, en cherchant à reproduire ce stigmate).

Un autre point est qu'il apparaît pratiquement impossible par percussion d'accrocher proprement le point d'origine d'une lamelle à détacher, si celui-ci n'est pas à la fois un tant soit peu en relief, et dégagé bilatéralement de façon à ce que le percuteur à face largement convexe n'accroche pas (trop) le bord du plan de frappe latéralement dans le même mouvement. On peut parer à cet incident (qui peut perturber l'initiation de la fracture de la lamelle, et/ou dégrader la surface de débitage) en préservant le sommet de l'angle formé par la troncature et le plan de frappe (cas

\begin{tabular}{|l|l|l|}
\hline Technique & dimensions des enlèvements & \multicolumn{1}{c|}{ critères qualitatifs indicatifs } \\
\hline & & \\
\hline Pression & $\begin{array}{l}\text { largeur 3 à } 8 \mathrm{~mm} \\
\text { longueur } 2,5 \text { à } 4,5 \mathrm{~cm}\end{array}$ & $\begin{array}{l}\text { o plusieurs enlèvements successifs alongés et minces } \\
\text { oxt. distale en "marche" (step-fract) } \\
\text { o corniche du (dernier) négatif intacte } \\
\text { onlèvement(s) oblique(s) }\end{array}$ \\
\hline & & \\
\hline Percussion & $\begin{array}{l}\text { largeur } 4 \text { à } 11+\mathrm{mm} \\
\text { longueur jusqu'à } 6,5 \mathrm{~cm}\end{array}$ & $\begin{array}{l}\text { o enlèvement s'élargissant } \\
\text { o ext. réfléchie ou en amorce de réfléchissement }\end{array}$ \\
\hline
\end{tabular}

Tableau 4 - Caractères des enlèvements expérimentaux détachés par pression et par percussion directe organique.

Table 4 - Characters of the experimental removals by pressure and by direct organic percussion. 

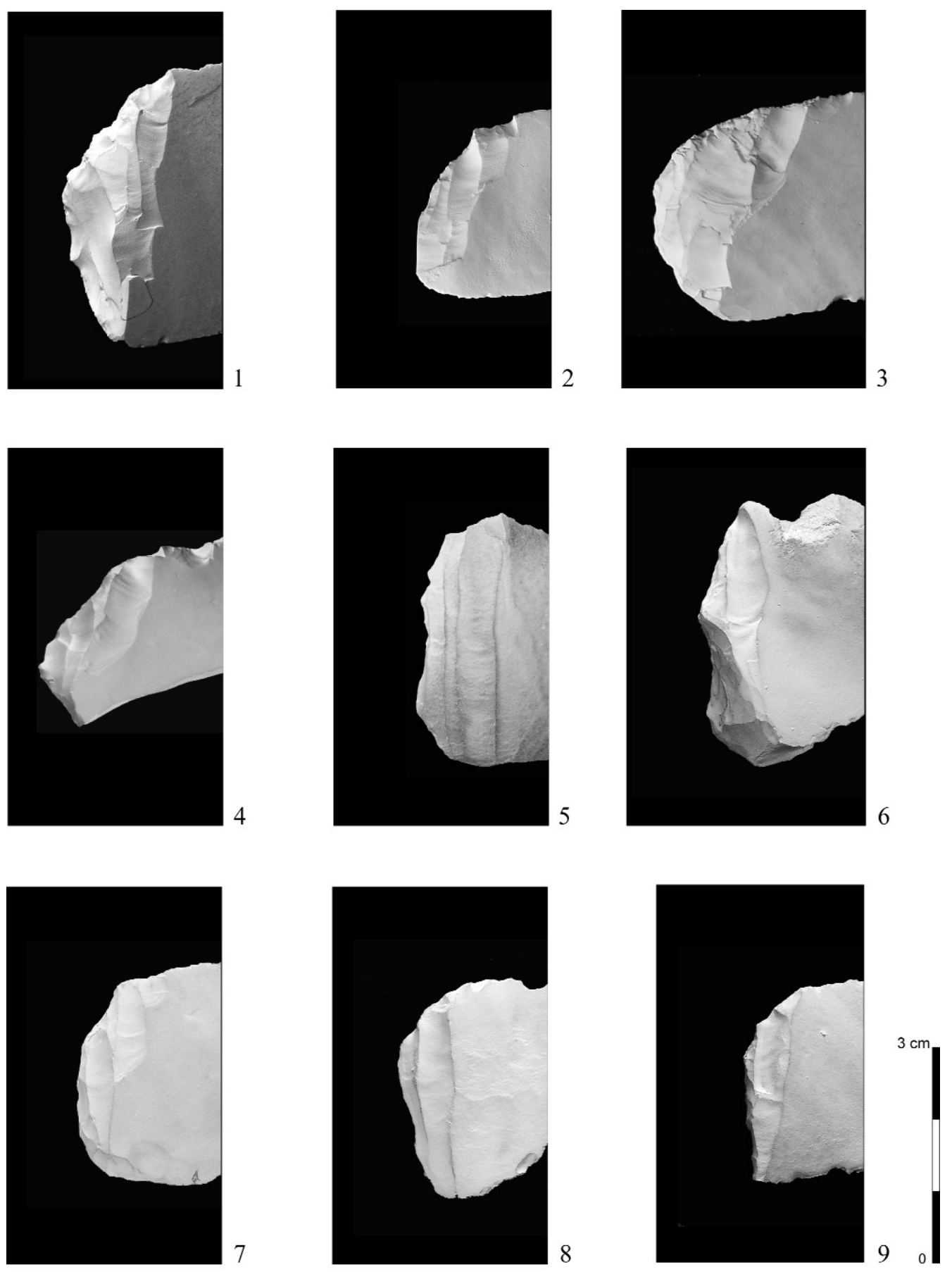

Figure 27 - Exemples de surfaces de débitage lamellaire archéologiques type «Bertonne » (n $n^{\circ s}$ 1-4:Trocadéro ; $n^{\circ} 5:$ La Rochette ; $n^{\circ s}$ 6-9 : La Bertonne, clichés L. Chehmana).

Figure 27 - Examples of archaeological bladelet production surfaces of « La Bertonne» type (n $n^{\text {s }}$ 1-4: Trocadéro; $n^{\circ}$ 5: La Rochette; nos 6-9: La Bertonne, photos by L. Chehmana). 


\section{Pression}
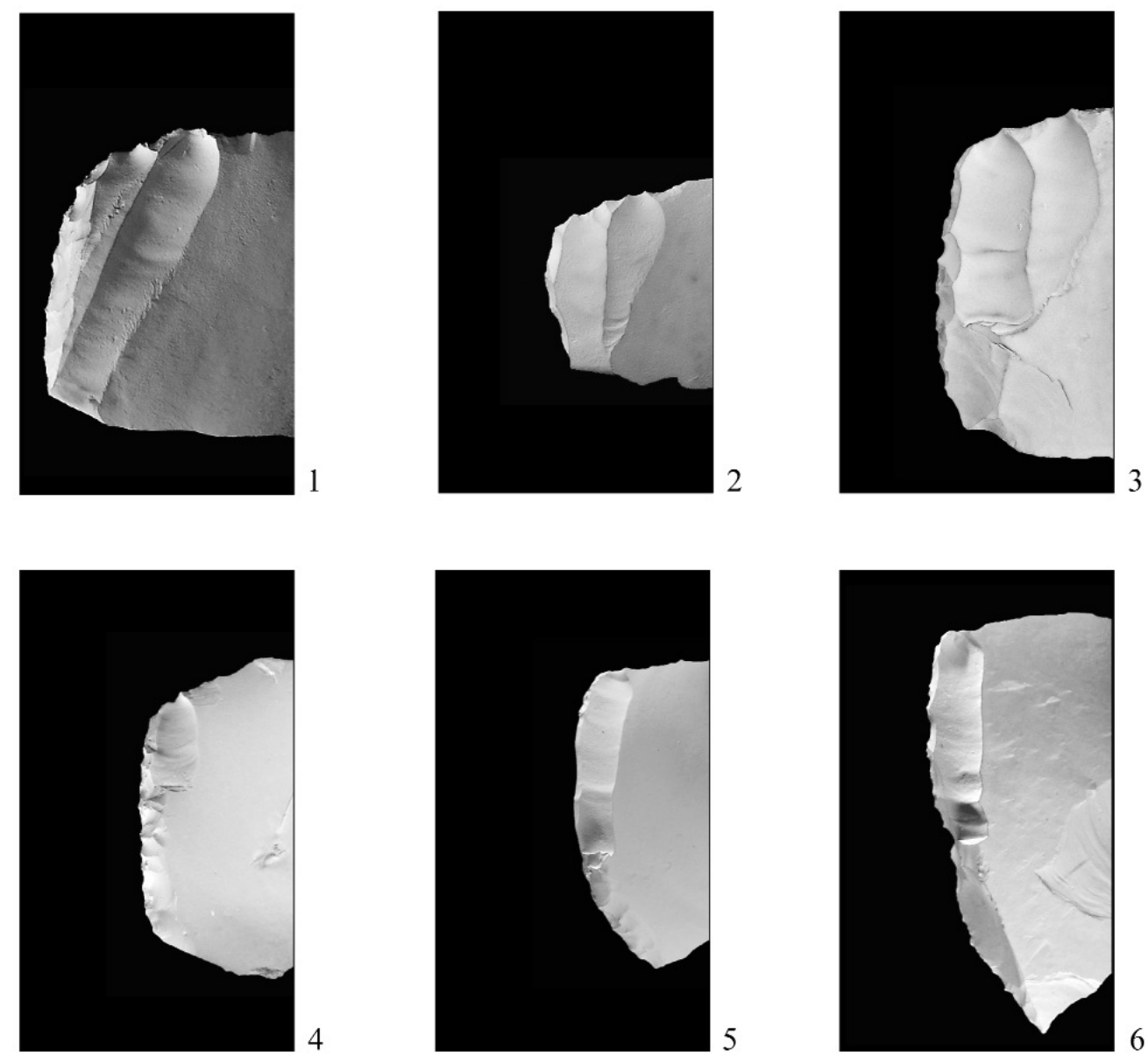

\section{Percussion}
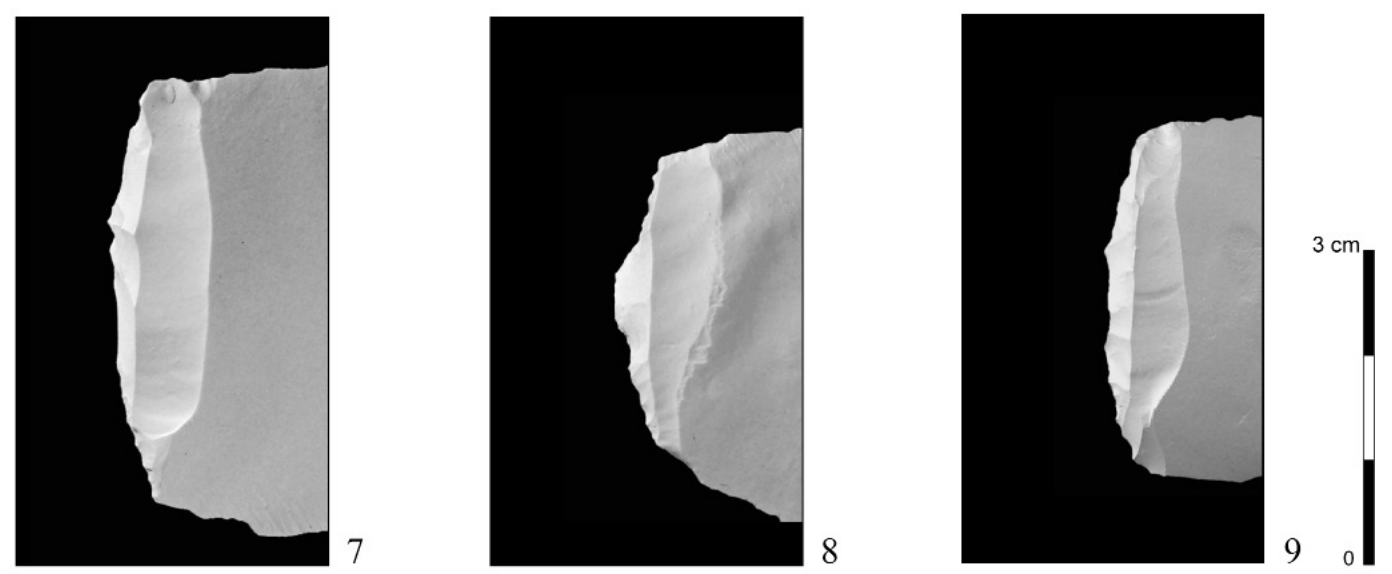

Figure 28 - Exemples de surfaces de débitage lamellaire expérimentales type «Bertonne » dont les enlèvements ont été effectués soit par pression (nos 1-6) soit par percussion (n $\left.{ }^{\circ s} 7-9\right)$, (clichés L. Chehmana, tests J. Pelegrin).

Figure 28 - Examples of experimental bladelet production surfaces of « La Bertonne » type using the pressure flaking technique ( $n^{\text {os }}$ 1-6) and percussion ( $n^{\circ \mathrm{s}} 7-9$ ), (photos by L. Chehmana, tests by J. Pelegrin). 
de la figure $28 \mathrm{n}^{\circ} 7$ et cas, assez général, sur les Orville). Cette contrainte est bien moindre pour la pression, qui peut être exercée par l'extrémité en pointe mousse d'un outil - ou pointe montée - en bois de cervidé, ce qui permet de placer cette pointe très précisément sur un microrelief du bord/plan de frappe sans avoir à dégager bilatéralement ce point de contact. De plus, la pression permet d'orienter la direction de la fracture, donnant au négatif une allure " déjetée " (cas de nombreux enlèvements d'aménagement de la troncature sur les pièces de la Bertonne : cf. dessins de pièces archéologiques) ou une direction franchement oblique. Ainsi, des séries d'enlèvements obliques tirés d'un bord en dévers, tel ceux de la fig. 27 nos 2 et 6 , sont très évocateurs de l'usage de la pression, et ont été reproduits selon cette technique (fig. $28 n^{\circ} 1$ ), la corniche du contre-bulbe du ou des enlèvements restant alors généralement intacte (fig. 28 nos 1 à 6 pour les pièces test par pression, fig. $27 n^{\circ s} 2$ et 6 pour les pièces archéologiques). En revanche, par percussion, cette corniche est souvent égratignée (fig. 28 nos 8 et 9 ), dès que le point de contact n'a pas été nettement dégagé.

Au vu de tous ces éléments (tabl. 4), il apparaît de façon assurée que la plupart des pièces de la Bertonne ont certainement été aménagées (troncature) et débitées par pression.

\section{Synthèse sur l'usage des pièces de la Bertonne comme nucléus à lamelles}

\section{Principe du débitage}

Le principe du schéma opératoire consiste à extraire une première lamelle par pression, sur la face inférieure d'un support (en général une lame), transversalement à son axe de débitage, à partir d'une nervure formée par l'alignement des extrémités distales d'une troncature inverse. Les nervures suivantes sont généralement exploitées, toujours par pression, et plusieurs lamelles, de gabarit variable, sont obtenues au cours d'une même séquence de production.

\section{Choix des supports de nucléus allié aux contraintes du débitage}

Pour extraire les lamelles, les tailleurs ont dans ce cas utilisé des lames particulièrement minces (fig. 29). Les supports ont une épaisseur moyenne de $8 \mathrm{~mm}$. Une certaine variabilité a toutefois été constatée puisque des lames et des éclats laminaires relativement plus robustes ont aussi été sélectionnés ; $30 \%$ mesurent entre 9 et $15 \mathrm{~mm}$ d'épaisseur dans la collection du Trocadéro mais il est rare qu'ils dépassent les $13 \mathrm{~mm}$ (fig. 29). Cette minceur relative paraît étonnante compte tenu de l'usage auquel étaient destinés ces supports. En effet, les nucléus sur produits débités ont souvent une épaisseur plus importante (cf. Le Brun Ricalens dir. 2005 pour l'Aurignacien ; Klaric 2003 pour le Gravettien ; Renard 2008 pour le Solutréen). Cela s'explique peut-être par le fait qu'ici les lamelles ont plus fréquemment été extraites sur la face inférieure du support que dans l'épaisseur.

Ainsi, la face inférieure des lames correspond à la surface de débitage des lamelles (fig. 30). Sa configuration respecte certaines règles pour que l'extraction des produits s'opère dans les meilleures conditions. La face inférieure des lames présente une légèrement convexité (fig. 30), autant dans l'axe transversal que dans l'axe longitudinal du support. Cette surface est généralement très régulière. Pour obtenir cette régularité, les tailleurs sélectionnent des lames dont le profil est de préférence globalement rectiligne. C'est probablement en partie pour cette raison que certains supports d'outils ont été recyclés. Quand ces conditions sont réunies sur la totalité du support, les tailleurs ont même exploité les deux extrémités des lames (fig. $19 \mathrm{n}^{\mathrm{os}} 2$ et 3 ).

Enfin, le plan de pression correspond à l'un des pans de la face supérieure du support. Ce pan n'a généralement pas été modifié. II forme un angle très aigu avec la face inférieure (fig. 30 et 31 ). Lorsque l'angle dépasse $50^{\circ}$, des modifications ont tout de même été effectuées : cette angulation est en fait souvent liée à la présence d'une nervure sur l'avers, à proximité du point de pression. L'angle est alors rectifié à l'aide d'une retouche directe (fig. 19 $n^{\circ} 4$; fig. $20 n^{\circ} 1$; fig. $21 n^{\circ s} 3$ et 4 ).

\section{Initialisation du débitage}

Pour initialiser le débitage, les tailleurs mettent en place une « nervure guide » par l'aménagement d'une troncature inverse. Plus précisément, c'est l'alignement progressif des extrémités distales d'une troncature inverse qui installe la « nervure guide ».

Les procédures d'installation varient en fonction de la morphologie de la section des lames. Les lames qui comportent deux à trois pans ont été privilégiées par les tailleurs. Dans ce cas, le procédé d'aménagement s'effectue en deux étapes. Les tailleurs aménagent dans un premier temps vers la face inférieure une retouche inverse, relativement courte (plutôt transversale à l'axe longitudinal du support), à partir du pan de la face supérieure qui est opposé au plan de pression d'où sont détachées les lamelles (fig. 32). Cette première ligne de retouche est alors recoupée par des enlèvements plus allongés (et plus obliques par rapport à l'axe longitudinal du support) qui alignent la « nervure guide » progressivement à l'aplomb du plan de pression.

Cette lecture avait déjà été effectuée par M. Lenoir (1987) qui avait également abordé la question de la technique utilisée pour la réalisation de cette troncature (fig. 32). D'après les expérimentations effectuées par l'un d'entre nous (J.-P.), l'agencement de ces enlèvements aurait été exécuté le plus souvent à l'aide d'un « presseur ». Cependant, la règle n'est pas stricte puisque certaines troncatures plus rectilignes (fig. 33) ont été parfois effectuées par percussion directe. Et finalement, nous soupçonnons l'usage conjoint des deux techniques dans plusieurs cas de figure. 
$\square$ Lames $\square$ Eclats laminaires

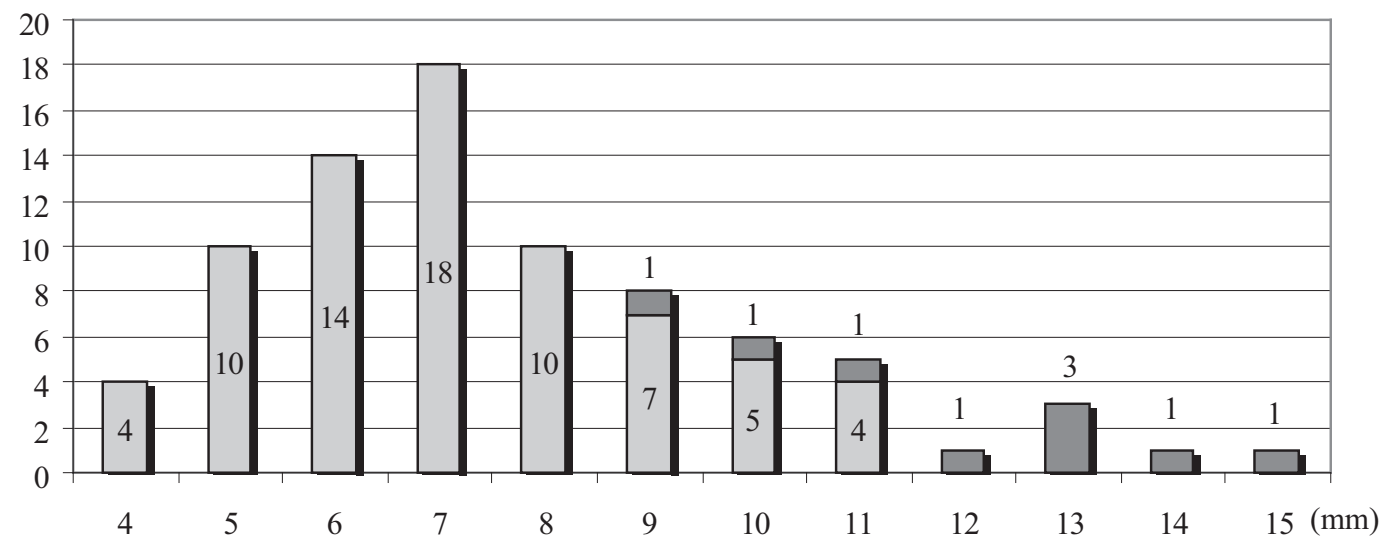

Figure 29 - Épaisseur des nucléus de la Bertonne du Trocadéro ( $N=76$, mesures prises sous l'emplacement des tables lamellaires).

Figure 29 - Thickness of La Bertonne cores from Trocadéro ( $N=76$, measurements made below the position of lamellar tables).
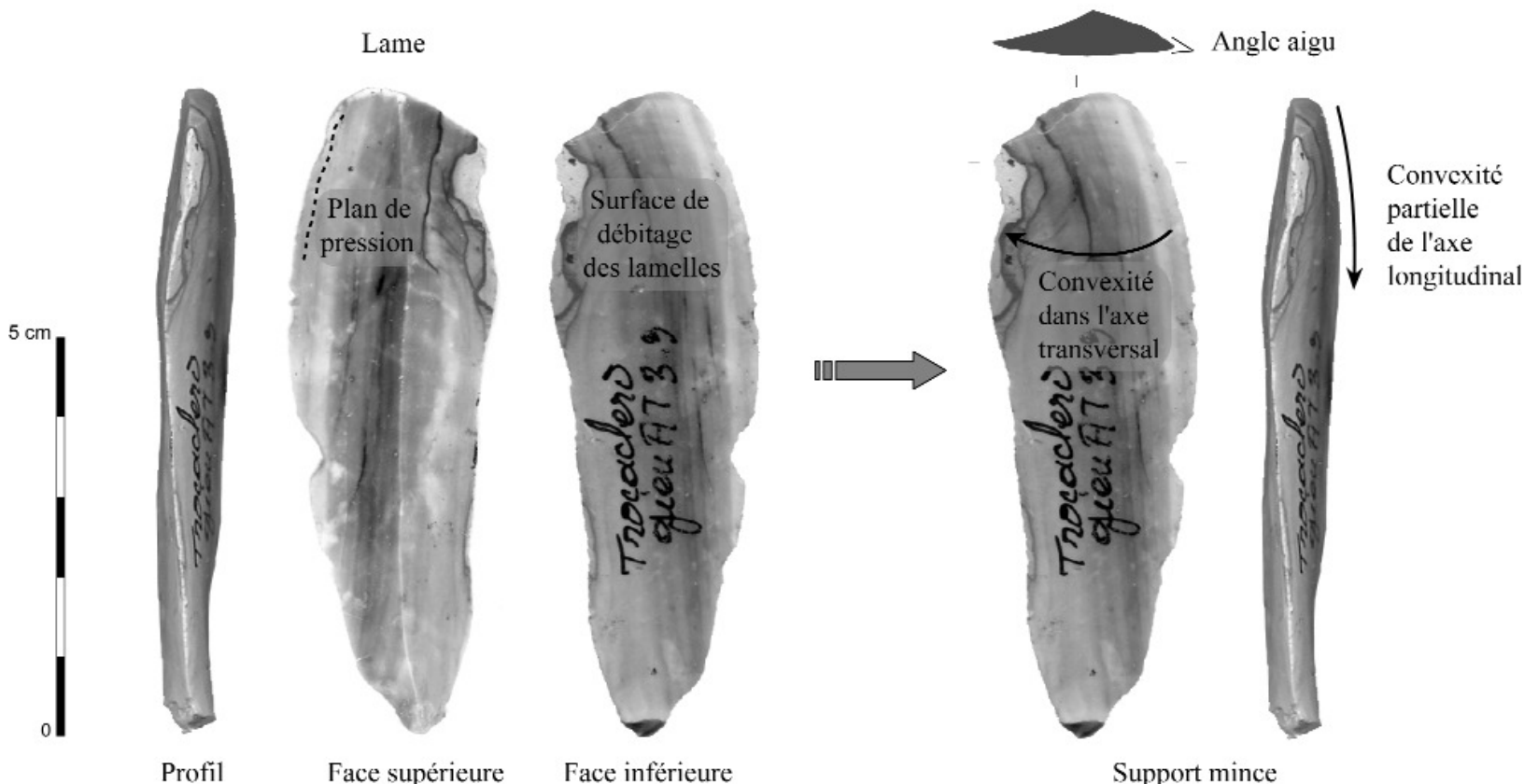

Figure 30 - Critères essentiels dans le choix des supports de nucléus (collection Bazin, site du Trocadéro, DAO L. Chehmana).

Figure 30 - Essential criteria for the choice of core supports (Bazin collection, Trocadéro site, DAO, L. Chehmana). 


\section{Latéralisation du plan de pression}

La latéralisation du plan de pression est une autre des particularités de ce débitage. Les lamelles sont pratiquement toujours extraites à partir du bord situé à droite de la troncature inverse lorsqu'elle est orientée vers le haut (autrement dit : du bord gauche du support si la troncature est distale, de son bord droit si la troncature est proximale). Dans la collection du Trocadéro, ce fait a été observé dans $100 \%$ des cas. Les seuls exemples où les Badegouliens semblent avoir dérogé à cette règle ont été constatés sur les sites où la matière première n'était pas disponible à proximité (comme à la Malignière et à Bellevue). Dans ce cas, le détachement des lamelles s'effectue à partir du bord opposé (il s'agit donc d'un débitage bipolaire) et toujours en fin de séquence de production. Cette exception à la règle, constatée à la Malignière et à Bellevue, s'explique peut-être par le fait que les tailleurs ont exploité au maximum les surfaces de débitage en fonction de la dernière possibilité qu'elles offraient.

Quoi qu'il en soit, la norme, au Trocadéro, était de positionner le plan de pression toujours du même côté de la troncature. Cette opération s'effectuait peut-être de façon mécanique, mais comment l'expliquer alors que les expérimentations - accomplies par P. Bodu et J. Pelegrin - ont montré qu'il était tout à fait possible de produire des

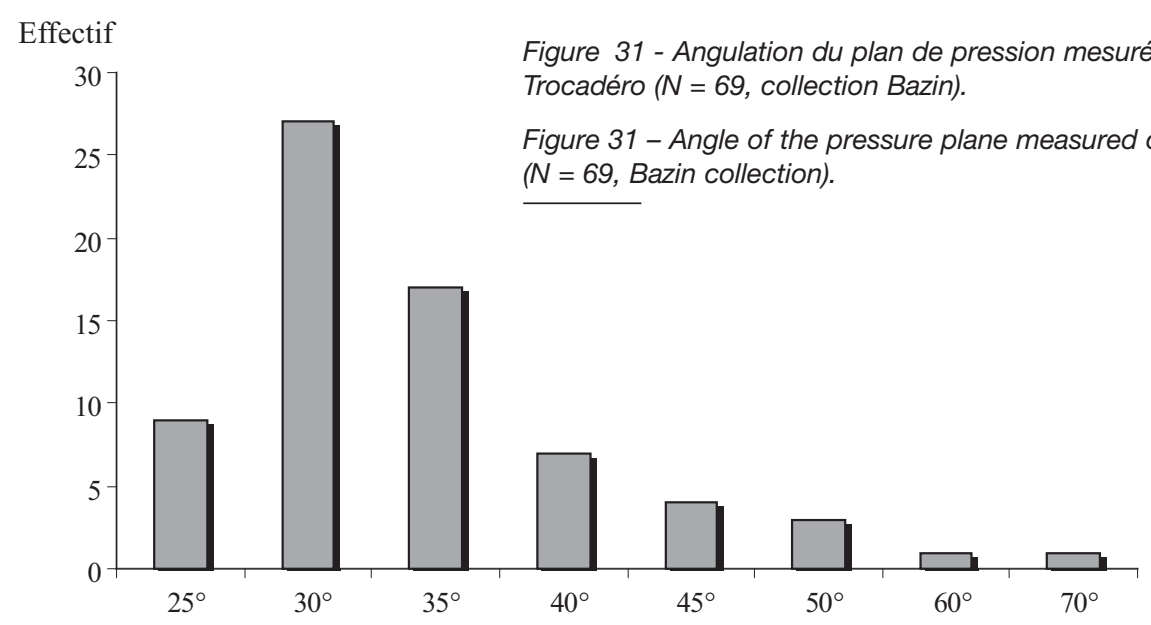

1. Retouches inverses courtes
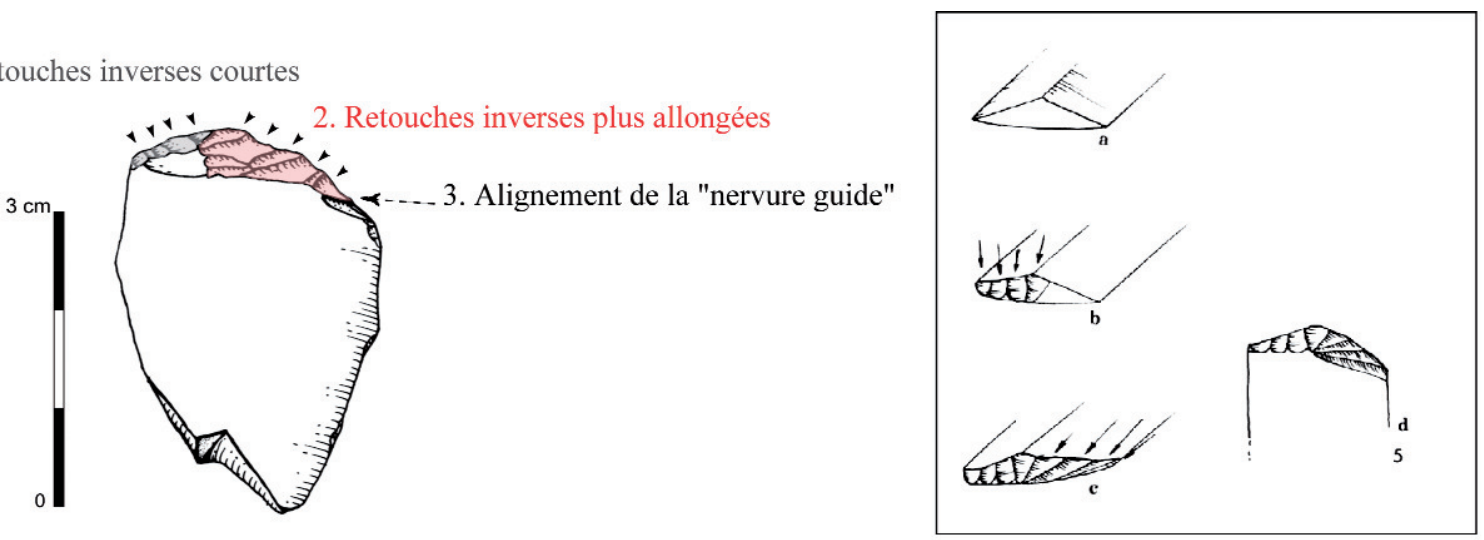

Pièce archéologique

Reconstitution schématique (Lenoir, 1987) (collection la Bertonne)

Figure 32 - Étapes de l'aménagement de la « nervure guide » (dessin L. Chehmana).

Figure 32 - Stages of preparation for the « guiding ridge » (drawings by L. Chehmana). 


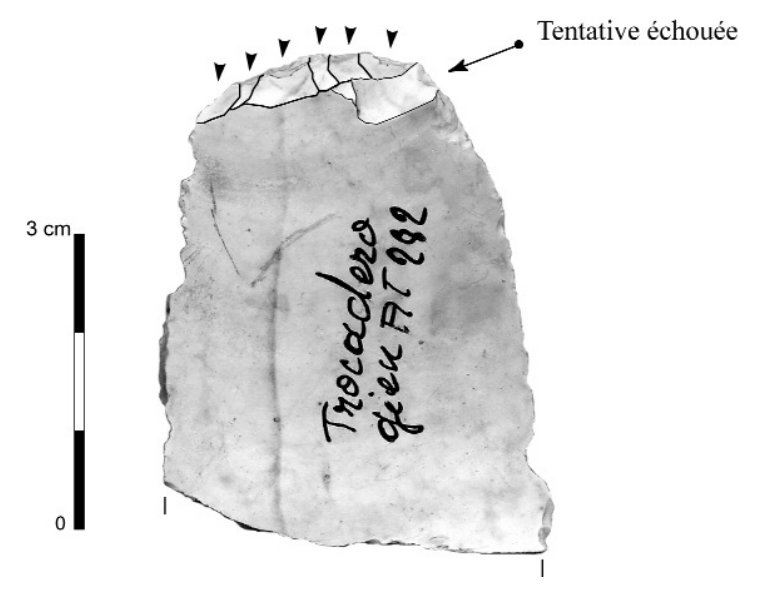

Figure 33 - Exemple d'une troncature inverse probablement réalisée par percussion (collection Bazin, site du Trocadéro, cliché L. Chehmana).

Figure 33 - Examples of an inverse truncation probably made by percussion (Bazin collection, Trocadéro site, photo by $L$. Chehmana).

lamelles identiques à partir de l'autre bord et sans contrainte supplémentaire (Pelegrin 1982 ; Perlès 1982). Selon l'un d'entre nous (J.P.), il semble que la position adoptée par les hommes préhistoriques soit plutôt appropriée pour les droitiers. Les gauchers, s'ils y étaient autorisés, étaient-ils alors forcés de pratiquer cette méthode à partir d'une règle imposée, stricte, ou bien existe-t-il une cause technique à cet automatisme ? C'est une question à laquelle nous aimerions pouvoir répondre car à notre connaissance, il n'existe qu'un seul autre cas où la latéralisation systématique que nous venons de décrire, associée à un débitage réalisé strictement dans l'axe transversal, a été constatée : il s'agit des débitages «Orville » (Perlès 1977, 1981 et 1982 ; Pelegrin 1982).

\section{Préparation au détachement}

Sur les pièces les mieux conservées, nous avons remarqué la présence d'esquillements de chaque côté du dernier négatif d'enlèvement lamellaire (fig. 17). Nous en avons conclu que la mise en relief du point de pression devait s'effectuer vers la face inférieure par des micro-enlèvements et probablement avec le même outil qui servait à détacher les lamelles.

\section{Déroulement du débitage}

Après le détachement de la première lamelle, deux nervures peuvent être exploitées pour guider l'extraction potentielle de deux autres lamelles. Une lamelle peut être obtenue à partir de la nervure qui recoupe la troncature, une autre à partir de la nervure qui recoupe la face inférieure. Cette dernière est parfois détachée sans que le plan de pression ne soit véritablement modifié. Toutefois, le plus souvent, les tailleurs reformatent la troncature, en l'abaissant progressivement jusqu'à l'emplacement du détache-

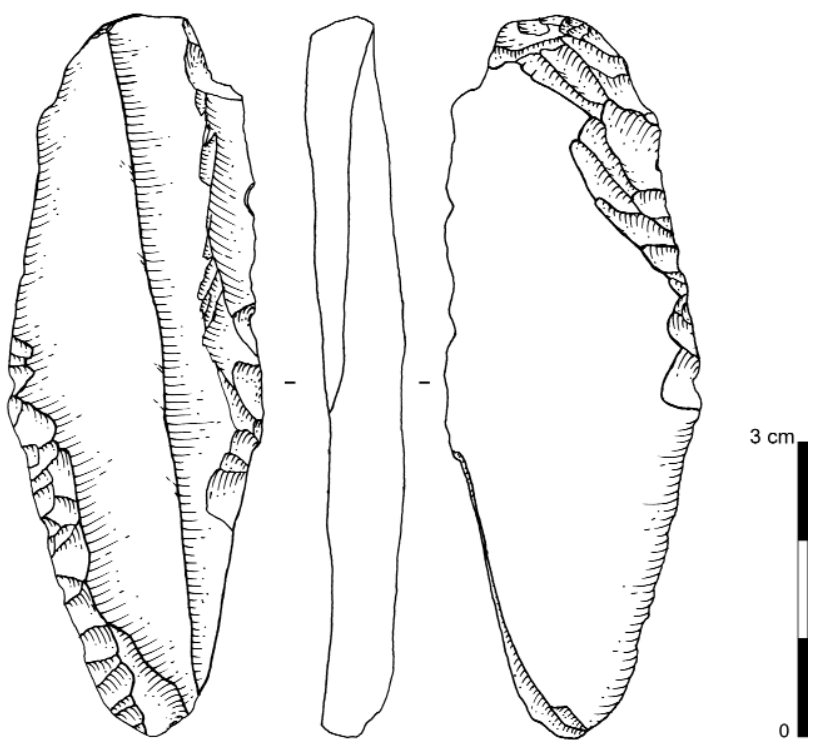

Figure 34 - Illustration de la progression du débitage sur un des nucléus provenant du site du Trocadéro (collection Bazin, dessin L. Chehmana).

Figure 34 - Illustration of the knapping sequence on one of the cores from the Trocadéro site (Bazin collection, drawing by L. Chehmana).

ment de la future lamelle. Cela permet de redonner une légère convexité à la surface de débitage mais aussi de rectifier l'alignement de la nervure.

Lorsque les convexités de la face inférieure le permettent, la progression du débitage se poursuit. Dans ce cas, l'orientation du détachement des lamelles évolue : au départ transversale à l'axe du débitage de la lame, elle s'incline progressivement (fig. 34). Cette progression est souvent rythmée par les réaménagements partiels de la troncature qui alignent les nervures suivantes jusqu'au plan de pression. À ce stade, il semble que se sont plutôt des lamelles relativement courtes qui ont été recherchées.

\section{Estimation du nombre de lamelles produites par séquence}

Un faible nombre de lamelles semblent avoir été produites lors de chaque séquence opératoire. D'après les nucléus les plus lisibles en fin d'exploitation (fig. 19, 20 et 21), nous estimons qu'une à quatre lamelles maximum ont été obtenues. Au vu des multiples négatifs d'enlèvements lamellaires présents sur certaines pièces (fig. 34), on pourrait en conclure que nous avons sous-estimé la production. Or, les surfaces de débitage sont systématiquement accidentées, ce qui signifie que certains enlèvements n'ont pas abouti à l'obtention d'un support satisfaisant. Le nombre de lamelles produites n'est donc pas égal au nombre de négatifs lamellaires. De plus, les quelques expérimentations qui ont été menées montrent que les lamelles détachées par pression se fracturent fréquemment au cours du détachement. 


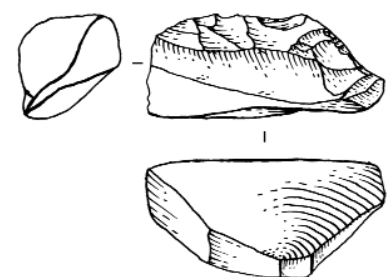

1

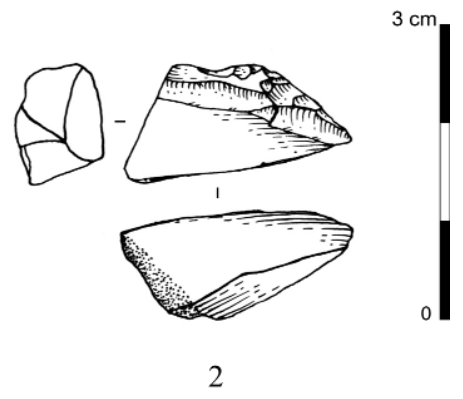

Figure 35 - Exemples de déchet produit lors de la réfection d'une table lamellaire (collection Lasnier, site de Bellevue et de la Malignière, dessins L. Chehmana).

Figure 35 - Examples of by-products produced during repreparation of a lamellar table (Lasnier collection, Bellevue and La Malignière sites, drawings by L. Chehmana).

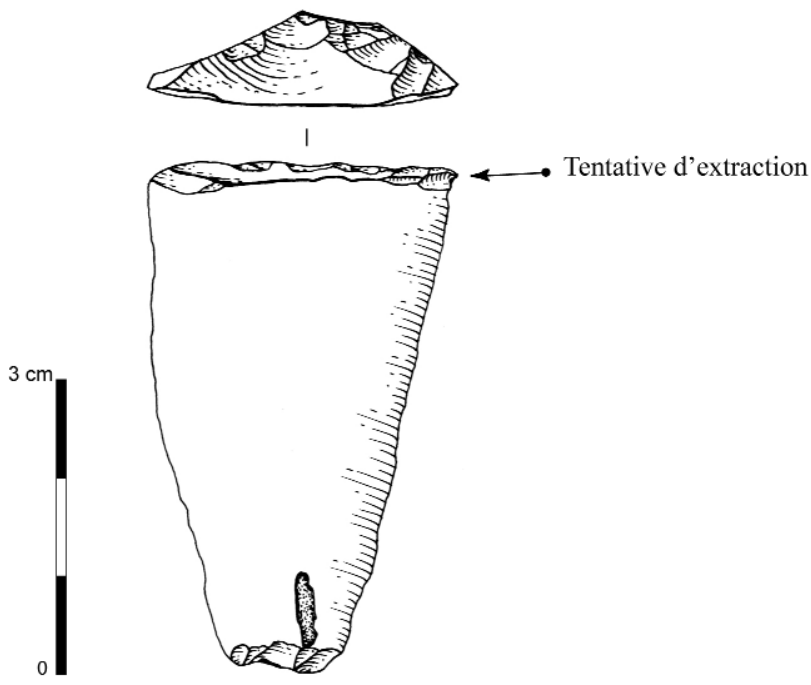

Figure 36 - Pièce de la Bertonne réaménagée après avoir été ravivée (collection Bazin, site du Trocadéro, dessin L. Chehmana).

Figure 36 - La Bertonne artifacts reshaped after having been reprepared (Bazin collection, Trocadéro site, drawing by L. Chehmana).

\section{Procédé de réinitialisation des séquences de production à partir du même support}

Si peu de lamelles sont produites par séquence, ces séquences peuvent se répéter plusieurs fois sur le même support. En effet, les pièces illustrées sur les figures 35 et 36 nous permettent d'évoquer une éventuelle réfection des nucléus. Les éclats de la figure 35 nous révèlent que les tailleurs ont parfois carrément tronqué la table lamellaire en portant un coup en retrait à partir de la face supérieure. La figure 36 illustre ce qui a été conservé, c'est-à-dire le support laminaire volontairement tronqué, comme par un " side-blow blade flake " (Inizan et al. 1995 - p. 88-89). L'arête située à la jonction de la face inférieure a été ensuite utilisée comme « nervure guide » pour initialiser la séquence de débitage.

Les troncatures ont sans doute été aussi réaménagées directement sur les tables lamellaires hors d'état. Ce procédé est cependant très peu reconnaissable sur les pièces archéologiques puisque les tailleurs suppriment progressivement la table lamellaire lorsqu'ils aménagent la nouvelle troncature. Une seule l'illustre : il s'agit d'une pièce provenant de la série éponyme de la Bertonne qui présente un négatif lamellaire qui est recoupé par une troncature inverse.

Effectif

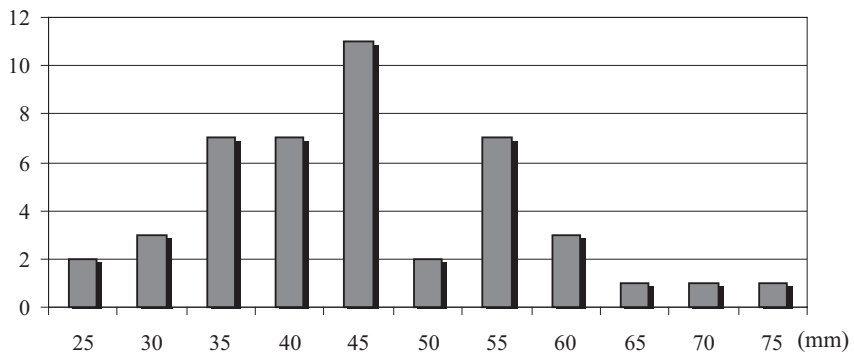

Figure 37 - Longueur résiduelle du support mesurée sur les pièces de la Bertonne du Trocadéro ( $N=45$, collection Bazin).

Figure 37 - Residual length of the support measured on La Bertonne artifacts from Trocadéro ( $N=45$, Bazin collection).

\section{Les raisons de l'interruption d'une séquence de production de lamelles}

L'interruption d'une séquence de production de lamelles est très souvent liée au manque de convexité transversale sur la surface de débitage. Ce manque de convexité entraîne des accidents qui empêchent la poursuite de la production. Les tailleurs abandonneront également les nucléus après une tentative échouée qui provoque de courts rebroussés au départ de l'enlèvement, parfois successifs lorsque le tailleur insiste un peu trop. Différentes raisons peuvent l'avoir provoqué : la mauvaise préparation du point de pression, un manque de force lors du détachement ou encore un angle du plan de pression trop ouvert.

Nous nous sommes par ailleurs demandés si les tailleurs n'avaient pas volontairement interrompu les débitages alors qu'ils avaient parfois la possibilité de poursuivre, au prix d'éventuelles réfections. Au Trocadéro, les lames n'ont pratiquement jamais été exploitées sur toute leur longueur (fig. 37). Ces parties auraient-elles été conservées afin de faciliter leur maintien lors du détachement des lamelles ? On pourrait même imaginer qu'elles aient pu être mainte- 
nues dans une gaine, comme cela a été proposé pour le détachement des lamelles par pression au Rocher-de-laCaille (Alix et al. 1995). Notons toutefois que sur certains sites, ces longueurs n'ont pas toujours été conservées. Les pièces sont parfois exploitées jusqu'à un stade beaucoup plus avancé (cf. la collection de la Malignière fig. 21). II est alors possible qu'en fonction des contextes d'approvisionnement, il ait existé des pratiques différentes pour le maintien des nucléus.

\section{Parmi les conséquences de la découverte...}

Découvrir qu'un « outil » préhistorique est en fin de compte un " nucléus à lamelles » est aujourd'hui assez banal pour le Paléolithique supérieur. On en voit par contre mieux l'intérêt lorsque l'on constate les répercussions que cette découverte a pu avoir sur la connaissance de la période durant laquelle les méthodes de taille ont été inventées. Pour celle qui nous concerne, deux répercussions importantes sont déjà prévisibles. On comprend maintenant pourquoi les pièces de la Bertonne nous sont apparues particulièrement similaires à celles d'Orville (fig. 38). Ayant finalement la même fonction, elles ont pu être comparées et révéler qu'il s'agissait de deux méthodes qui ont été régies par le même principe de débitage (Chehmana 2009 et thèse en cours). Ainsi, le principe du débitage Orville exposé par C. Perlès (1982) et J. Pelegrin (1982) dans les années quatre-vingt, est également valable pour le débitage Bertonne. Dans les deux cas, « les tailleurs ont cherché à détacher une lamelle [initiale] à partir d'une arête positionnée à la rencontre d'une troncature inverse et de la face inférieure d'une lame ». Leur ressemblance n'est donc pas liée à une simple convergence de forme. II s'agit bien là d'une véritable parenté technique.

\section{NUCLEUS "BERTONNE"}

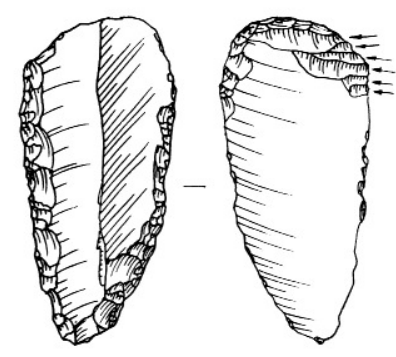

1

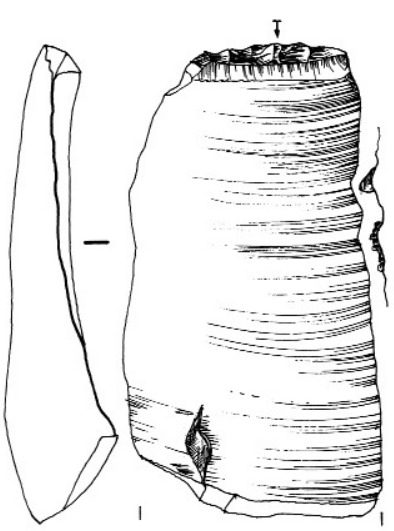

4

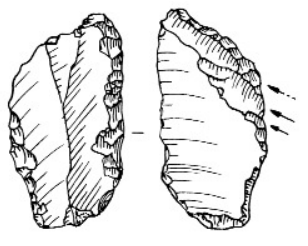

2

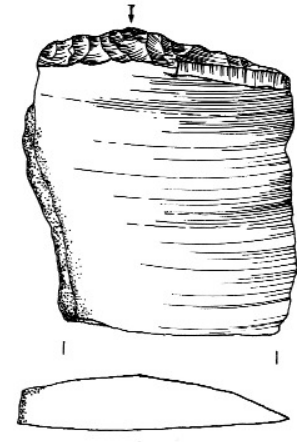

5

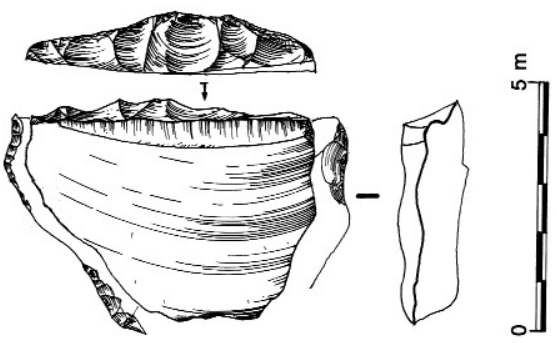

6

Figure 38 - Exemplaires de nucléus de la Bertonne (collection Daleau, d'après dessins M. Lenoir dans Lenoir, 1983 fig. 203) et d'Orville (collection Perlès, d'après dessins A. Moundrea dans Perlès, 1982 fig. 1 et fig. 2).

Figure 38 - La Bertonne cores (Daleau collection, after draxings by M. Lenoir in Lenoir 1983, fig. 203) and Orville cores (Perlès collection, after drawings by A. Moundrea in Perlès 1982, fig. 1 and 2). 
Nous irons jusqu'à émettre l'hypothèse que ces deux méthodes font partie d'une même lignée et que l'une d'entre elles est peut-être l'amélioration technique de l'autre. Cette proposition implique que les groupes qui les ont conçues ont certainement vécu à une période relativement proche dans le temps. En ce qui concerne la sériation des industries, cela signifie que nous rapprochons l'industrie d'Orville - alors placée, sans grande certitude, dans la phase moyenne du Magdalénien - de celle de la Bertonne attribuée au Badegoulien.

\section{Remerciements}

Nous tenons à remercier tout particulièrement B. Valentin pour avoir " redécouvert » la série du Trocadéro et ces fameuses pièces de la Bertonne. Nous le remercions également, ainsi que G. Debout, d'avoir relu attentivement cet article.

Nos remerciements vont aussi à J.-P. Daugas et J.-P. Bracco qui nous ont autorisé à citer la date inédite du Blot.

Merci également à $F$. Hubert (conservateur du Musée d'Aquitaine), V. Mistrot et M. Lenoir de nous avoir permis d'examiner la série de la Bertonne. II aura fallu plus de six mois pour retrouver celles de la Malignière et de Bellevue, conservées à l'heure actuelle au dépôt archéologique de Guéret. Pour leur aide précieuse dans la recherche de ces collections, nous remercions la conservatrice, C. Vax et l'assistante de conservation du Musée de Guéret, ainsi que P.-Y. Demars, F. Trotignon et P. Leger, actuellement responsable des collections de monsieur Lasnier. Un grand merci également à la conservatrice du Musée d'Evreux, L. Le Cieux, à l'attaché de conservation du Musée d'Evreux, G. Leblond, à D. Cliquet, conservateur régional de BasseNormandie et au petit-fils de M. Coutil, pour nous avoir aidés à recueillir des informations sur la série de la Caboche. Enfin merci à C. Duchadeau-Kervazo, C. Chauchat et M. Dachary pour les précisions qu'ils nous ont apportées au sujet des séries du Signal et de Azkonzilo et à J.-C. Merlet pour les articles qu'il nous a très gentiment transmis sur les séries de Seyresse et du Réservoir.

\section{Références bibliographiques}

ALIX J., PELEGRIN J. et DELOGE H. 1995 - Un débitage de lamelles par pression au Magdalénien de Rocher-de-laCaille (Loire, France). PALEO, 7, Décembre 1995, S.A.M.R.A., Les Eyzies, p. 187-199.

ARAMBOUROU R. 1970 - Un campement protosolutréen à Seyresse. Bull. Soc. Borda, p. 1-9.

BAYLE DES HERMENS R. (de) 1979 - Les niveaux supérieurs du Magdalénien de la grotte du Rond-du-Barry, Polignac (Haute-Loire). In : D. de Sonneville-Bordes (Éd.), La fin des temps glaciaires en Europe. Chronostratigraphie et écologie des cultures du Paléolithique final. Talence, colloque international (du 24-28 mai 1977), Paris, CNRS édition, p. 601-611.
BODU P., CHEHMANA L., DEBOUT G., BIGNON O., en préparation - Le gisement badegoulien de Oisy (Nièvre). Supplément à Gallia Préhistoire.

BODU P., DEBOUT G. et TABORIN Y. 2005 - De la parure chez les Badegouliens du Bassin parisien. Le cas du site de Oisy dans la Nièvre. In : V. Dujardin (Éd.), Industrie osseuse et parures du Solutréen au Magdalénien en Europe. Angoulême, table ronde (28-30 mars 2003). Mémoire XXXIX de la Société préhistorique française, p. 87-99.

BORDES F. 1958 - Nouvelles fouilles à Laugerie-Haute Est : premiers résultats. L'Anthropologie, 62, p. 205-244.

BOSSELIN B. et DJINDJIAN F. 1988 - Un essai de structuration du Magdalénien français à partir de l'outillage lithique. Bulletin de la Société préhistorique française, 85, p. 304-331.

BRACCO J-P. 1992 - Le Paléolithique supérieur du Velay et ses abords. Recherches sur la dynamique des peuplements et l'occupation du sol dans un milieu volcanique de moyenne montagne. Aix-en-Provence : Université de Provence - Aix-Marseille I. Thèse de Doctorat.

BRACCO J.-P., MORALA A., CAZALS N., CRETIN C., FERULLO O., FOURLOUBEY C., LENOIR M. 2003 - Peuton parler de débitage discoïde au Magdalénien ancien/Badegoulien ? Présentation d'un schéma opératoire de production d'éclats courts normalisés. In : M. Peresani (Ed.), Discoid lithic tecchnology : advances and implication. British archaeological Reports, International Series 1120, Oxford, p. 83-116.

BREZILLON M.N. 1968 - La dénomination des objets de pierre taillée. Matériaux pour un vocabulaire des préhistoriens de langue française, Paris, CNRS (Suppléments à Gallia Préhistoire IV), 413 p.

BRICKER H. M. 1995 - Le Périgordien moyen de l'abri Pataud niveau 5. In : Bricker H.M. (dir.). Le Paléolithique supérieur de l'abri Pataud (Dordogne) : les fouilles de H.L. Movius Jr. DAF, Ed. de la Maison des Sciences de I'Homme, Paris, p. 133-165,

BRICKER H.M. et DAVID N. 1995 - Le Périgordien VI de l'abri Pataud niveau 5. In : Bricker H.M. (dir.). Le Paléolithique supérieur de l'abri Pataud (Dordogne) : les fouilles de H.L. Movius Jr. DAF, Ed. De la Maison des Sciences de l'Homme, Paris, p. 89-104,

BROOKS A.S. 1995 - L'Aurignacien de l'abri Pataud niveaux 6 à 14. In : Bricker H.M. (dir.), Le Paléolithique supérieur de l'abri Pataud (Dordogne) : les fouilles de H.L. Movius Jr. DAF, Ed. De la Maison des Sciences de I'Homme, Paris, p. 167-225,

CAZALS N. 2000 - Constantes et variations des traits techniques et économiques entre le Magdalénien "inférieur" et "moyen" : analyse des productions lithiques du nord de la péninsule ibérique. Paris : Université de Paris-PanthéonSorbonne. Thèse de doctorat, 2 vol., 586 p. 
CAZALS N. 2005 - Le début du Magdalénien de part et d'autre des Pyrénées. Quelques réflexions au travers des techniques de taille et des modes d'exploitation des ressources. In : J. Jaubert et M. Barbaza (Eds.), Territoires, déplacements, mobilité, échanges pendant la Préhistoire. Terres et hommes du Sud. Actes du 126 $6^{\mathrm{e} m e}$ Congrès National des Sociétés Historiques et Scientifiques, Toulouse, avril 2001, p. 295-309.

CHALARD P. 1993 - Solutréen supérieur et Badegoulien du gisement des Peyrugues à Orniac (Lot). Essai d'individualisation de deux niveaux en contact, par le biais de l'analyse techno-économique des industries lithiques. Toulouse : Université Toulouse II - le Mirail. Mémoire de DEA, École des Hautes Etudes en Sciences Sociales, 162 p.

CHAMPAGNE F., ESPITALIÉ R. 1981 - Le Piage, site préhistorique du Lot. Paris : Mémoires de la Société préhistorique française, 15, $205 \mathrm{p}$.

CHAUCHAT C. (dir.) 1999 - L'habitat magdalénien de la grotte du Bourrouilla à Arancou (Pyrénées-Atlantiques). Gallia Préhistoire, 41, p. 1-151.

CHEHMANA L. 2009 - The conception of "Bertonne" and "Orville » bladelet production (Upper Paleolithic, France). Human Evolution, vol. 24, n 1, p. 71-79.

CHEHMANA L. sous presse - La méthode Orville : une invention badegoulienne? Soumis au Bulletin de la Société préhistorique française.

CHEHMANA L., thèse en cours - Réponses culturelles à la crise climatique du Dernier Maximum Glaciaire. Approche techno-économique comparée des industries lithiques entre Solutréen et Magdalénien (titre non définitif).

CHEHMANA L., BODU P. 2005 - Le peuplement du Bassin parisien durant le Dernier Maximum glaciaire : bilan des connaissances sur la culture matérielle des groupes humains au Badegoulien et au Magdalénien ancienmoyen, In P. Bodu et L. Chehmana dir., Le Paléolithique supérieur ancien au centre et au sud du Bassin parisien : " des systèmes techniques aux comportements ", Rapport de PCR dans le cadre du programme P4, Région CentreNord, Paris, SRA Île-de-France, p. 57 à 100.

CHEYNIER A. 1930 - Un outil magdalénien nouveau en silex à Badegoule : la raclette. Bulletin de la Société préhistorique française, 27, p. 483-488.

CLEYET-MERLE J.-J. 1992 - Le Magdalénien de la vallée de la Couze et ses origines d'après les fouilles des Jamblancs. In : J.-P. Rigaud, H. Laville, B. Vandermeersch (dir.), Le Peuplement magdalénien : paléogéographie physique et humaine. Colloque de Chancelade (10-15 octobre 1988). Paris, Éd. du CTHS, p. 223-234.

CLOTTES J. et GIRAUD J.-P. 1989 - Le gisement préhistorique du Cuzoul (Vers, Lot). Quercy-Recherche, 65-66, juinsept. 1989, p. 82-91.
CRETIN C. 1996 - Vers une nouvelle perception du Badegoulien des Jamblancs. Premiers éléments technoéconomiques. PALEO, 9, p. 243-268.

CRETIN C. 2000 - Tradition et variabilité dans le comportement technique. Le cas du Badegoulien et du Magdalénien en Périgord. Paris : Université de Paris I-PanthéonSorbonne. Thèse de doctorat, $451 \mathrm{p}$.

DACHARY M. 2002 - Le Magdalénien des Pyrénées occidentales. Nanterre : Université de Paris X-Nanterre. Thèse de Doctorat, 2 vol., 299 p.

DALEAU F. 1909 - Silex à retouches anormales de la station de la Bertonne ou La Rousse, commune de Peujard (Gironde). Soc. Achéol. de Bordeaux, XXXI, p. 31-48, 5 fig., 8 pl.

DALEAU F. 1911 - Encore les silex à retouches anormales. In : compte rendu de la $39 \mathrm{e}$ session du congrés de I'A.F.A.S., Toulouse, 1910, notes et mémoires, 2, Paris, Ed. A.F.A.S. p. $275-276$.

DAUGAS J.-P. et DELPORTE H. (†) (dir.) et alii - Le site du Paléolithique supérieur (Périgordien, Gravettien et Magdalénien) de I' Abri du Blot, à Cerzat (Haute-Loire). Monographie collective en cours de préparation.

DELPORTE H. 1968 - Crozant. La Malignière. Circonscription d'Auvergne et Limousin. Gallia Préhistoire, 11 , p. $439-441$.

DEMARS P.-Y. 1973 - Le gisement aurignacien de la Bombetterie, commune de Cublac (Corrèze). Essai de comparaison morphologique avec l'outillage aurignacien de Chanlat et Bos del Ser. Bulletin de la Société préhistorique française, 70, Etudes et Travaux, p. 311-323.

DEMARS P-Y. 1985 - La station de plein air magdalénienne de la Malignière, commune de Crozant (Creuse). In : B. Lasnier (Éd.). Crozant, études archéologiques. Documents d'Archéologie Creusoise, 1, p. 27-39.

DEMARS P.-Y., LAURENT P. 1989 - Types d'outils lithiques du Paléolithique supérieur en Europe. Cahiers du Quaternaire, 14, Paris, CNRS, 178 p.

DETRAIN L. et coll. 1994 - Fouilles du Musée National de Préhistoire des Eyzies. Rapport final.

DUCASSE S. et LANGLAIS M. 2008 - Interprétation technologique et discussion autour du statut culturel des " pièces de la Bertonne ", l'exemple de la série lithique de Seyresse (Landes, France). PALEO, 20, p. 59-88.

DUCHADEAU-KERVAZO C. 1982 - Recherches sur l'occupation paléolithique dans le bassin de la Dronne. Bordeaux : Université de Bordeaux I. Thèse de Doctorat en Géologie du Quaternaire et Préhistoire, 939 p.

EVIN J., MARIEN G., PACHIAUDI Ch. 1975 - Lyon Natural Radiocarbon Measurements V., Radiocarbon, 17, 1, p. 4-34. 
EVIN J., MARIEN G., PACHIAUDI Ch. 1976 - Lyon Natural Radiocarbon Measurements VI., Radiocarbon, 18, 1, p. 60-88.

EVIN J., MARIEN G., PACHIAUDI Ch. 1978 - Lyon Natural Radiocarbon Measurements VII., Radiocarbon, 20, 1, p. 19-57.

FOURLOUBEY C. 1996 - La production de raclettes au Châtenet (Saint-Front de Pradou, Dordogne). PALEO, 8, p. 269-275.

HEMINGWAY M.H. 1980 - The Initial Magdalenian in France. BAR International series, 90, 2 vol., 502 p.

INIZAN M.-L., REDURON M., ROCHE H. 1995 Technologie de la pierre taillée. Éd. CREP, Préhistoire de la pierre taillée 4, $199 \mathrm{p}$.

KLARIC L. 2003 - L'unité technique des industries à burins du Raysse dans leur contexte diachronique. Réflexions sur la diversité culturelle au Gravettien à partir des données de la Picardie, d'Arcy-sur-Cure, de Brassempouy et du Cirque de la Patrie. Paris : Université de Paris I. Thèse de Doctorat, $426 \mathrm{p}$.

KLARIC L., AUBRY T., WALTER B. 2002 - Un nouveau type d'armature en contexte gravettien et son mode de production sur les burins du Raysse (la Picardie, commune de Preuilly-sur-Claise), Bulletin de la Société préhistorique française, 99, 4, p. 751-764.

LANGLAIS M. 2007 - Dynamiques culturelles des sociétés magdaléniennes dans leurs cadres environnementaux. Enquête sur 7000 ans d'évolution de leurs équipements lithiques entre Rhône et Ebre. Toulouse : Université de Toulouse II et Barcelone. Thèse de Doctorat, $550 p$.

LE BRUN RICALENS F. (coord. avec la collaboration de Bon F. et Bordes J.-G.) 2005 - Productions lamellaires attribuées à l'Aurignacien : chaînes opératoires et perspectives technoculturelles. Actes du $14^{e}$ Congrès de I'Union internationale des sciences préhistoriques et protihistoriques. Liège, 2001, Luxembourg, Musée national d'histoire et d'art (Archéologiques, 1), 568 p.

LENOIR M. 1976 - Etude typologique et technologique des " pièces à retouches anormales » de la station de la Bertonne, commune de Peujard (Gironde). Bulletin de la Société préhistorique française, 73, p. 43-47.

LENOIR M. 1983 - Le Paléolithique des basses vallées de la Dordogne et de la Garonne. Bordeaux : Université de Bordeaux I. Thèse de Doctorat d'Etat ès Sciences, 2 vol., $702 \mathrm{p}$.

LENOIR M. 1987 - La pièce de la Bertonne, « fossile directeur » du Magdalénien ancien ? Bulletin de la Société préhistorique française, 84, 6, p. 167-171.

LENOIR M. 2000 - La fin des temps glaciaires dans les basses vallées de la Dordogne et de la Garonne. In : G. Pion (dir.), Le Paléolithique supérieur récent: nouvelles données sur le peuplement et l'environnement, Actes de la
Table ronde de Chambéry, 12-13 mars 1999, Paris, Editions de la Société préhistorique française, (Mémoire de la Société préhistorique française, XXVIII), p. 81-87.

LEROI-GOURHAN A. et BRÉZILLON M. 1972 - Fouilles de Pincevent : essai d'analyse ethnographique d'un habitat magdalénien (la section 36). Paris, CNRS Éditions, (Suppl. à Gallia Préhistoire, VII), 345 p.

LEYSSALLES G. et NOONE H.V.V. 1949 - Le Pech de Saint-Sourd. L'Anthropologie, 53, p. 247-251.

MERLET J.-C. 2005 - Le Badegoulien et le Magdalénien ancien dans le Bassin de l'Adour : un état de la question. Documents d'archéologie des Pyrénées atlantiques, 24, p. 103-118.

MORALA A. 1993 - Technologie lithique du Magdalénien ancien de l'abri Casserole (Les Eyzies-de-Tayac, Dordogne). PALEO, 5, p. 193-208.

MOVIUS H.L. 1995 - Inventaire analytique des sites aurignaciens et périgordiens de Dordogne. In : Bricker H.M. (dir.). Le Paléolithique supérieur de l'abri Pataud (Dordogne) : les fouilles de H.L. Movius Jr. DAF, Ed. de la maison des Sciences de I'Homme, Paris, p. 227-313.

PARISOT O. 1995 - Analyse typo-technologique du matériel lithique du site d'Orville (Indre, France). Mémoire de Maîtrise, Université Paris X-Nanterre, 83 p.

PELEGRIN J. 1982 - Approche expérimentale de la technique de production lamellaire d'Orville. In : D. Cahen (Éd.), Tailler! pour quoi faire, Studia Praehistorica Belgica, p. $149-158$

PERLÈS C. 1977 - Note préliminaire sur un type d'outil particulier du site d'Orville (Indre). Bulletin de la Société préhistorique française, t. 74, 5, p. 141-144.

PERLÈS C. 1981 - Quelques réflexions sur la typologie lithique à propos des pièces d'Orville (Indre). Préhistoire Africaine, Mélanges offerts au Doyen Lionel Balout. Éd. A.D.P.F., Paris, p. 367-373.

PERLÈS C. 1982 - Les "outils d'Orville" : des nucléus à lamelles. In : D. Cahen (Éd.), Tailler ! pour quoi faire, Studia Praehistorica Belgica, p. 129-148.

PEYRONY D. et E. 1938 - Laugerie-Haute, près des Eyzies. Instit. Paléonto. Hum., Mémoire 19, 84 p., 58 fig., 7 pl.

PEYRONY D. 1949 - Le Périgord préhistorique : essai de géographie humaine. Périgueux : Soc. hist. arch. Périgord, $92 \mathrm{p}$.

PRIMAULT J., GABILLEAU J., BROU L., LANGLAIS M., GUERIN S. et coll. 2007 - Le Magdalénien inférieur à microlamelles à dos de la grotte du Taillis des Coteaux à Antigny (Vienne, France). Bulletin de la Société préhistorique française, 104,1 , p. 5-30.

RENARD C. 2008 - Les premières expressions du Solutréen dans le Sud-Ouest français. Évolution techno- 
économique des équipements lithiques au cours du dernier maximum glaciaire. Paris : Université de Paris X-Nanterre. Thèse de doctorat, $448 \mathrm{p}$.

RIGAUD J.-P. 1986 - Peujard. La Bertonne. Aquitaine. Gallia Préhistoire, 29, 2, p. 241.

SACCHI D. 1976 - Les civilisations du Paléolithique supérieur en Languedoc occidental (Bassin de l'Aude) et en Roussillon. In : H. de Lumley (Éd.), La Préhistoire française, Les civilisations paléolithiques et mésolithiques de la France. Nice, 1976, IX Congrès de I'UISPP. Tome I, p. 1174-1188 (Paris, Éd. du CNRS).

SACCHI D. 2003 - Retour sur le Badegoulien de Lassac (Aude), Mélanges Jean Gaussen, Bulletin de la société préhistoriques Ariège-Pyrénées, LVIII, 2003, p. 157-162.

SERONIE-VIVIEN M-R. avec la collaboration de Benoist F., Boulestin B., Bourhis J-R., Duday H., Guadelli J-L., Le Gall O., Joannidès N., Marinval P., Marquet J-C., Martin H., Solari M-E., Vernet J-L. 1995 - La grotte de Pégourié, Caniac-du-Causse (Lot). Préhistoire Quercinoise, supplément $n^{\circ} 2,334 \mathrm{p}$.

SMITH P.E.L. 1966 - Le Solutréen en France. Bordeaux (France) : Delmas (Publication de l'Institut de Préhistoire de l'Université de Bordeaux, 5) : 449 p.
SONNEVILLE-BORDES D. 1960 - Le Paléolithique supérieur en Périgord. Bordeaux : Delmas, 2 vol., 560 p.

TROTIGNON F. 1985 - La station paléolithique de surface de Bellevue. In : B. Lasnier (Éd.), Crozant, études archéologiques. Documents d'Archéologie Creusoise, 1, p. 41-48.

TROTIGNON F., POULAIN T., LEROI-GOURHAN A. 1984 Etude sur l'abri Fritsch (Indre). XIX'̀me supplément à Gallia Préhistoire, CNRS Éd., 122 p.

VALENTIN B. 1995 - Les groupes humains et leurs traditions au Tardiglaciaire dans le Bassin parisien : apports de la technologie comparée. Paris : Université de ParisPanthéon-Sorbonne. Thèse de Doctorat de Préhistoire de l'Université de Paris I, 3 vol., 1106 p.

VIGNARD E. 1965 - Le Badegoulien. Bulletin de la Société préhistorique française, 62, 8, p. 262-263.

VIALOU D. (dir.) 2004 - La Préhistoire. Histoire et Dictionnaire. Éd. Robert Laffont, 1627 p. 